\title{
Annual Meeting of the International Academy of Cardiovascular Sciences: North American Section
}

\author{
3rd Cardiovascular Forum for Promoting Young Investigators and \\ Centers of Excellence in Cardiovascular Research
}

\author{
September 10 to 12, 2015 • Omaha, Nebraska, USA
}

\section{Keynote Address}

\begin{abstract}
001
MOLECULAR BASIS FOR THE BENEFICIAL EFFECTS OF $\mathrm{CO}_{2}-$ WATER BATH THERAPY IN PERIPHERAL ARTERY DISEASE

\section{NS Dhalla}

Institute of Cardiovascular Sciences, St Boniface Hospital

Research, Faculty of Health Sciences, University of Manitoba,

Winnipeg, Manitoba

A wide variety of factors are considered to participate in the pathogenesis of peripheral artery disease (PAD) and different agents are being used for the treatment of this major health problem. Although reduction in blood flow due to narrowing of arteries in the ischemic limb is the major cause of $\mathrm{PAD}$, none of the therapeutic interventions are satisfactory. We investigated the effects of $\mathrm{CO}_{2}$-water bath (CWB) therapy on blood flow in the ischemic hind limb. The femoral artery was occluded in rats to induce PAD and the animals were treated with or without $\mathrm{CWB}$ at $37^{\circ} \mathrm{C}$ for 4 weeks (20 min/day; 5 days/week) starting one week after artery occlusion. Peaks, mean and minimal blood flows were not detected in the untreated ischemic hind limb of animals due to arterial ligation. However, blood flow values were about $50 \%$ of the control upon treatment with CWB; $67 \%$ of the ligated animals showed positive blood flow by $\mathrm{CO}_{2}$ treatment. Morphological examination of the treated ischemic skeletal muscle revealed a 3 -fold increase in small artery numbers indicating the formation of new blood vessels. Extensive studies have revealed that $\mathrm{CO}_{2}$ at low concentrations serves as a redox sensitive signal transduction molecule for the genesis of angiogenesis. It is suggested that beneficial action of $\mathrm{CO}_{2}$ therapy on blood flow to hind limb may be due to the development of angiogenesis in the ischemic skeletal muscle.
\end{abstract}

\section{Session 2.1: Cerebrovascular Diseases and Stroke}

\section{2}

\section{LARGE AND SMALL CEREBROVASCULAR DISEASE DURING HYPERTENSION}

FM Faraci

Carver College of Medicine, University of lowa, lowa City, lowa, USA Vessel disease has a major impact on human health and is particularly devastating for brain. Hypertension is a major risk factor for vascular disease and a key cause of stroke and cognitive deficits. In the cerebral circulation, hypertension produces diverse effects on structure and function. Underlying mechanisms involve interacting oxidative- and inflammatoryrelated processes that produce inward vascular remodeling, loss of collateral vessels, hypoperfusion, and impairment of adaptive responses that regulate cerebral blood flow (eg, endothelium-dependent vasodilation and neurovascular coupling). The renin-angiotensin system (RAS) is a major therapeutic target in patients with essential and some other forms of hypertension. Although activation of local or tissue RAS is thought to be a key cause of hypertension, the impact of tissue RAS on the local vasculature is poorly understood. Recent work demonstrates that activation of the central RAS with deoxycorticosterone (DOCA)-salt (which simultaneously suppresses the peripheral RAS) profoundly alters the cerebrovasculature.
Endothelial function in cerebral arteries is markedly impaired by DOCA-salt whereas dilation of mesenteric arteries is normal. These vascular effects extend throughout the microcirculation and include parenchymal arterioles where NO-mediated signaling is substantially impaired. In relation to mechanisms, microvascular changes were mediated by local activation of AT1 and mineralocorticoid receptors as well as Rho kinase. Along with activation of RAS components in the cerebral cortex, DOCA-salt increased expression of RAS elements in the cerebrovasculature. Thus, in addition to direct effects of hypertension, the cerebral circulation may also be impacted by local RAS activation. Such findings may help explain why the impact of hypertension is so profound in brain.

\section{3}

\section{THE EXERCISING BRAIN IN HEALTH AND DISEASE} WG Mayhan, DM Arrick

Department of Cellular Biology and Anatomy, and the Cardiovascular Center, Louisiana State University Health Sciences Center - Shreveport, Shreveport, Louisiana, USA

Exercise training has been shown to be beneficial for reducing the risk of premature death and disease, including cardiovascular- and cerebrovascularrelated diseases. The mechanisms that account for the protective effect of exercise training appear to be related to activation/inhibition of many diverse cellular networks. Our studies have concentrated on an examination of the influence of exercise training on several aspects of cerebrovascular function during health and disease. First, we examined reactivity of cerebral arterioles in response to eNOS- and nNOS-dependent agonists in sedentary and exercise-trained animals. We found that moderate exercise training did not influence reactivity of cerebral arterioles in normal animals, but restored impaired responses of cerebral arterioles observed during disease states (diabetes and smoking). The mechanism for the protective influence of exercise training on cerebral arterioles during disease states appeared to be related to an alteration in NOS and/or oxidative stress. Second, we examined whether exercise training could alter brain injury following cerebral ischemia/ reperfusion. We found that moderate exercise training did not influence infarct volume in normal animals, but lessened brain injury during disease states. Finally, we wondered whether vigorous exercise training, as compared to moderate exercise, could improve cerebrovascular function and prevent brain injury during normal physiologic conditions. We found that vigorous exercise training increased NOS-dependent reactivity of cerebral arterioles and reduced infarct volume following cerebral ischemia/reperfusion. Based upon our findings, we suggest that exercise training is a viable therapeutic approach for the prevention of cerebrovascular disease during physiologic and pathophysiologic conditions.

\section{4}

\section{A NEW DIETARY APPROACH FOR THE CONTROL OF HYPERTENSION}

GN Pierce ${ }^{1,2}$, AL Edel ${ }^{1,2}$, S Caligiuri ${ }^{1,2}$, H Aukema ${ }^{1,3}$, A Ravandi ${ }^{1,4}$, E Dibrov $^{1,2}$, W Weighell ${ }^{1,5}$, R Guzman ${ }^{1,5}$, D Rodriguez-Leyva ${ }^{1,2}$, M Aliani ${ }^{1,3}$

${ }^{1}$ Canadian Centre for Agri-food Research in Health and Medicine (CCARM), St Boniface Hospital; Departments of ${ }^{2}$ Physiology and Pathophysiology, ${ }^{3}$ Human Nutritional Sciences, ${ }^{4}$ Internal Medicine, ${ }^{5}$ Surgery, University of Manitoba, Winnipeg, Manitoba

Hypertension is an important silent killer and the leading global risk for burden of death in the world. Current medications used to control hypertension 
are costly, can induce unwanted side-effects and they are not always effective in controlling blood pressure (BP) in all hypertensive patients. Having a food that will control BP represents an alternative strategy that is more popular amongst patients than drugs. Flaxseed is enriched in the cardioprotective omega-3 fatty acid (alpha linolenic acid (ALA)), lignans and fiber. The FlaxPAD Trial was initiated to determine if dietary supplementation with milled flaxseed in hypertensive peripheral artery disease (PAD) patients could provide beneficial actions. The clinical population was randomized into a ground flaxseed or whole wheat placebo group. Individuals were required to consume $30 \mathrm{~g}$ of the appropriate intervention which was incorporated into different foods daily. This FlaxPAD Trial was double-blinded and involved 110 patients. Both brachial and central systolic and diastolic blood pressures were significantly reduced in the flaxseed group relative to control following the year-long intervention. Both total and LDL cholesterol levels were reduced by flaxseed by about $10-15 \%$. The mechanisms for these effects will be discussed. We conclude that consuming ground flaxseed daily may offer a significant dietary strategy to lower both circulating cholesterol and BP.

Supported by CIHR, Flax2015, ARDI, Western Grains Research Foundation, SaskFlax and St Boniface Hospital Foundation.

\section{Session 2.2: Inflammation, Cytokines and Cardiovascular Disease}

\section{5}

\section{INTRACRINE ANG II FUNCTION ORIGINATES FROM NON-CANONICAL PATHWAYS IN THE HUMAN HEART CM Ferrario \\ Wake Forest University School of Medicine Winston Salem, North Carolina, USA}

Increased cardiac Ang II expression and activity contributes to cardiac hypertrophic remodeling, arrhythmias, and fibrosis. Our research suggests that renin angiotensin system (RAS) inhibitors are less effective in blocking Ang II-mediated adverse cardiac remodeling because its production in cardiac myocytes follows a non-canonical pathway through the processing of the intermediate substrate angiotensin-(1-12) [Ang-(1-12)] by chymase. While in the rat angiotensin converting enzyme (ACE) hydrolyzes Ang(1-12) into Ang II, chymase but not ACE converts Ang-(1-12) directly into Ang II in human heart tissue. The human form of cardiac $\alpha$-chymase, either expressed in cardiomyocytes or incorporated into these cells from activated mast cells and fibroblasts, is the enzyme producing Ang II directly from Ang-(1-12). The importance of this non-canonical pathway comprised by the chymase/Ang-(1-12)/Ang II axis is illustrated by the demonstration of an increased expression of Ang-(1-12) and chymase in the left atrial appendage of patients undergoing open heart surgery for the treatment of ischemic heart or left valvular disease. In summary, Ang II-mediated adverse cardiac remodeling and arrhythmogenesis is a result of the intracellular activation of a chymase/Ang-(1-12)/Ang II axis that is not amenable to blockade by current RAS blockers. Furthermore, these biotransformation steps are species-specific.

\section{7}

\section{CASPASE-1, AN INNATE IMMUNE SENSOR IN ENDOTHELIAL CELLS}

X-F Yang

Temple University School of Medicine, Philadelphia,

\section{Pennsylvania, USA}

The role of receptors for endogenous metabolic danger signals-associated molecular patterns (DAMPs) has been characterized recently as bridging innate immune sensory systems for DAMPs to initiation of inflammation. However, it remains unknown whether endothelial cells (ECs), the cell type with the largest numbers and the first vessel cell type exposed to circulating DAMPs in the blood, can sense hyperlipidemia using caspase-1/ inflammasome as innate immune sensor. We performed three interconnected projects: First, using bone marrow transplantation, and atherogenic
ApoE-/-/caspase-1-/- double knockout mice, we found that early hyperlipidemia promotes EC activation before monocyte recruitment via a caspase-1-sirtuin 1-activator protein-1 pathway, which provides an important insight into the development of novel therapeutics for blocking caspase-1 activation as early intervention of metabolic cardiovascular diseases, inflammations and atherosclerosis. Second, we found that caspase-1 activation inhibits vascular endothelial growth factor receptor 2 expression whereas caspase-1 inhibition improves the tube formation of proatherogenic lipid lysophosphatidylcholine-treated human ECs; and Caspase-1 depletion improves angiogenesis and blood flow in mouse hind-limb ischemic tissues. Our results have demonstrated that inhibition of proatherogenic caspase-1 activation in ECs improves angiogenesis and the prognosis of ischemic diseases including myocardial infarction (MI), peripheral arterial disease, ischemic stroke, etc. Finally, using gene knockout mice, cell therapy and MI model, we found that hyperlipidemia activates caspase- 1 in Sca-1+ endothelial progenitor cells (EPC), which subsequently weakens Sca-1+ EPC repair of vascular EC injury. Our results have demonstrated the therapeutic potential of caspase-1 inhibition in improving progenitor cell therapy for MI.

\section{8}

\section{INFLAMMATION AS A SOURCE OF SYMPATHETIC EXCITATION IN HEART FAILURE \\ RB Felder}

Carver College of Medicine, University of Iowa, Iowa City, Iowa, USA The past decade has seen an increasing appreciation for the role of inflammation in cardiovascular disease states. In systolic heart failure, circulating levels of pro-inflammatory cytokines correlate directly with the severity of illness and portend a poor prognosis. Augmented sympathetic nervous system activity is another predictor of adverse outcomes in heart failure, promoting increased preload and afterload on the failing heart, impaired heart function, and cardiac arrhythmias. My laboratory has examined the effect of inflammation on central nervous system mechanisms driving sympathetic nervous activity in heart failure. We have found that the prototypical pro-inflammatory cytokines - tumor necrosis factor-alpha (TNF-a) and interleukin-1 beta (IL-1b) - that increase in the plasma of humans with severe heart failure, activate central neural circuits that drive sympathetic nerve activity in a rat model that mimics heart failure after myocardial infarction in humans. A small region of the brain that lacks a blood-brain barrier - the subfornical organ - plays a major role in transmitting these circulating signals of peripheral inflammation to the brain, where they act to increase sympathetic drive and to facilitate the sympatho-excitatory influence of other circulating signals. We have also found that TNF-a and IL-1b increase early after myocardial infarction and are sustained at high levels inside the blood brain barrier in the hypothalamic paraventricular nucleus, an important cardiovascular-related nucleus that regulates sympathetic nerve activity. I will discuss the mechanisms by which these central inflammatory influences augment sympathetic nerve activity in heart failure and the potential for therapeutic interventions in the central inflammatory state.

\section{Session 2.3: Cardiac and Vascular} Regeneration

\section{9}

\section{CELL THERAPY FOR ISCHEMIC CARDIOMYOPATHY}

R Bolli

University of Louisville, Division of Cardiovascular Medicine, Institute of Molecular Cardiology, Louisville, Kentucky, USA

Cell-base therapies offer enormous potential for the treatment of ischemic cardiomyopathy. Among them, c-kit+ cardiac stem cells (CSCs) have emerged as extremely promising. Numerous studies from many labs have shown that CSCs improve post-infarction LV dysfunction and remodeling in animal models. Most transplanted CSCs disappear in a few days; their most likely mechanism of action is production of paracrine factors that 
beneficially affect the adjacent myocardium. We conducted SCIPIO, the first human study of CSCs. SCIPIO was a Phase I, randomized, open-label trial of c-kit+ CSCs in patients with post infarction LV dysfunction (EF $<40 \%$ ). In the twenty treated patients, LVEF (3D Echo) increased from $29.2 \pm 1.9 \%$ before CSCs to $37.8 \pm 2.9 \%$ at 1 year $(\mathrm{P}<0.001)$ and $38.1 \pm 3.6 \%$ at 2 years $(\mathrm{P}=0.004)$. This was associated with improved quality of life (Minnesota Living with Heart Failure Questionnaire [MLHFQ]: $45.3 \pm 4.3$ vs. $22.2 \pm 5.4$ and $19.5 \pm 4.2$ at 1 year and 2 years, respectively $[\mathrm{P}<0.001])$ and improved NYHA class ( $\mathrm{P}<0.001$ at 1 and 2 years). In contrast, in the thirteen controls there was no improvement. In the treated patients who underwent MRI, infarct size decreased by $43.2 \%$ at 1 year $(\mathrm{P}<0.001)$ and $39.4 \%$ at 2 years $(\mathrm{P}=0.008)$. Thus, a single infusion of $\mathrm{CSC}$ s was sufficient to improve ischemic cardiomyopathy for at least 2 years. These results suggest that infusion of autologous CSCs in patients with ischemic heart failure is safe and that its beneficial effects are sustained and actually increase over time.

\section{0}

\section{GROWTH FACTOR EFFECTS ON CARDIAC STEM CELLS}

AM Johnson, KG Aghila Rani ${ }^{1}$, CC Kartha

Rajiv Gandhi Center for Biotechnology and ${ }^{1}$ Sree Chitra Tirunal Institute for Medical Sciences \& Technology, Trivandrum, India

Clinical utility of stem cells derived from the adult heart is hindered by lack of sufficient cell numbers. Designing strategies favoring activation and migration of resident stem cells to the site of injury are an exciting frontier of research in regenerative medicine. We have explored two avenues in this direction. We studied the effect of growth factors in inducing cardiosphere formation from cardiac stem cells (CSCs) isolated from atrial biopsies obtained from patients who underwent coronary artery bypass surgery. Among the different growth factors analyzed, EGF significantly promoted cardiosphere formation and proliferation of cardiosphere derived cells. Our findings suggest that EGF could be used for generating CDCs which could provide ample cell numbers for transplantation therapies. In a postnatal mammalian heart, cardiac stem cells exist in a quiescent state. Disrupting quiescence could induce proliferation and differentiation in these cells. We investigated the role of $\mathrm{FoxO} 3 \mathrm{a}$ in promoting quiescence in c-kitpos cardiac stem cells. FoxO3a is highly expressed in quiescent stem cells isolated from neonatal Balb/C mice hearts. Igfr-1 mRNA expression was markedly reduced in these cells. Regulation of FoxO3a by mitogens was studied in vitro using a 'reserve cell' model in which actively dividing cells were switched to mitogen restricted medium. This causes the cells to become either terminally differentiated or enter a quiescent phase. Our studies reveal that IGF-1 is a regulator of FoxO3a and FoxO3a regulates the transcription of Kip family proteins such as p27kip1 and p57kip2. An up-regulation of p27kip1 and p57kip2 transcripts was observed in growth factor starved CSCs which also had increased FoxO3a expression. Igf-1 stimulation significantly declined the expression of FoxO3a, p27kip1 and p57kip2 transcripts and increased the expression of Cyclin D1 mRNA. In CSCs, it may be possible that Igf-1 inactivates FoxO3a leading to down-regulation of $\mathrm{p} 27 \mathrm{kip} 1$ and $\mathrm{p} 57 \mathrm{kip} 2$ and promotes cell-cycle progression. Our findings add to the growing knowledge of molecular mechanisms related to quiescence and proliferation of cardiac stem cells.

The studies were funded by Department of Biotechnology, Government of India

\section{1}

AN APPRAISAL OF BONE MARROW CELL THERAPY: METAANALYSIS AND MORE

R Afzal, A Samanta, V Jeevanantham, $\underline{B}$ Dawn

Division of Cardiovascular Diseases, Cardiovascular Research Institute, Midwest Stem Cell Therapy Center, University of Kansas Medical Center, Kansas City, Kansas, USA

A number of clinical trials of bone marrow cell (BMC) therapy for heart repair have been completed over the past decade. These relatively small trials used different types of BMCs injected through various routes in highly variable numbers in patients with a wide range of clinical conditions and prognosis. The results from these studies have been therefore somewhat variable. Furthermore, the conclusions of several meta-analyses performed on dissimilar datasets from various clinical trials have also been divergent. Nonetheless, a critical review of a number of these meta-analyses suggests that BMC therapy indeed enhances left ventricular parameters compared with standard therapy in patients with ischemic heart disease. Although the magnitudes of benefits are numerically small, the favorable impact BMC therapy on clinical outcomes seems substantial. However, several important variables need to be optimized before the full potential of BMC therapy can be delivered to patients. Continued and careful analysis of data from completed and ongoing trials may provide critical insights to improve outcomes of bone marrow cell therapy in the future.

Supported in part by NIH grant R01 HL-117730.

Session 2.4: The Grant Pierce
Young Investigator Competition
in Cardiovascular Sciences

\section{3}

\section{DIVERGENT EFFECTS OF PRO-INFLAMMATORY CYTOKINES ON VEGF-A-INDUCED DIFFERENTIATION OF MESENCHYMAL STEM CELLS INTO ENDOTHELIAL CELLS}

IA Ikhapoh, CJ Pelham, DK Agrawal

Department of Medical Microbiology and Immunology and Center for Clinical and Translational Science, Creighton University School of Medicine, Omaha, Nebraska, USA

Coronary artery stenting/angioplasty procedures frequently result in complications including endothelial loss and arterial thrombosis. Mesenchymal stem cells (MSCs) have therapeutic potential during cardiac interventions. We examined the effects of the pro-inflammatory cytokines, IL-6, TNF $\alpha$ and Ang II on VEGF-A-stimulated differentiation of Yucatan microswine bone marrow derived MSCs into endothelial cells (ECs). Naïve MSCs were immunonegative for classical EC markers. Low-dose VEGF-A $(2 \mathrm{ng} / \mathrm{ml})$ modestly increased immunopositivity for EC markers (cells $v W F+16 \pm 2 \%$, PECAM- $1+13 \pm 4 \%$, and VE-cadherin $+11 \pm 7 \%$; $<<0.05$ vs. naïve MSCs). High-dose VEGF-A ( $50 \mathrm{ng} / \mathrm{ml})$ induced greater immunopositivity for EC

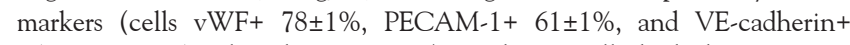
$65 \pm 1 \% ; p<0.05$ vs. low-dose VEGF-A). Mechanistically, high-dose VEGF-A treatment of MSCs significantly upregulated mRNA expression of S0x18 (2.0 \pm 0.2 -fold; 24 hours), a transcription factor that programs EC differentiation. The siRNA-mediated knockdown of Sox 18 greatly reduced VEGF-Amediated induction of EC markers. CO-stimulation of MSCs with Ang II $(2 \mathrm{ng} / \mathrm{ml})$ and low-dose VEGF-A caused a synergistic increase in the percentage of cells immunopositive for EC markers to similar levels as high-dose VEGF-A alone, whereas Ang II alone had no effect. In contrast, treatment of MSCs with IL-6 or TNF $\alpha$ induced a dose-dependent decrease in EC marker expression in the presence of high-dose VEGF-A. However, further supplementation of IL- 6 or TNF $\alpha$ and VEGF-A with Ang II rescued the expression of EC markers. These changes in EC marker expression positively correlated with capillary tube formation and expression of Sox 18 mRNA and protein. Our findings highlight the opposing effects of pro-inflammatory cytokines on EC differentiation and have important clinical implications towards cardiac interventions.

\section{4}

MITOCHONDRIAL- DEPENDENT APOPTOSIS IN ARTERIAL REMODELING: LINK TO HYPERTENSION

A Familtseva, A Kalani, P Chaturvedi, S Pushpakumar, N Metreveli, SC Tyagi

Department of Physiology and Biophysics, University of Louisville, Louisville, Kentucky, USA

Hyperhomocysteinemia (HHcy) has been observed to promote hypertension through endothelial dysfunction and vascular remodeling, but the mechanisms are unclear. Previously, we showed that elevated homocysteine levels facilitated excessive mitochondrial fission with consequent endothelial cell loss and collagen deposition in the mesenteric artery. In the present study, we hypothesize that HHcy-induced excessive mitochondrial fragmentation promotes mitochondrial apoptosis through Bax activation that up-regulates 
downstream apoptotic proteases (Caspase-9, Caspase-3), causing endothelial cell loss and arterial remodeling that predispose to hypertension. To test this hypothesis, we used 12 week old C57BL/6J mice (WT) as a control; Cystathionine- $\beta$-synthase deficient mice $(\mathrm{CBS}+/-)$ with genetic HHcy; $\mathrm{C} 3 \mathrm{H} / \mathrm{HeJ}(\mathrm{C} 3 \mathrm{H})$ mice, that are resistant to oxidative stress and $\mathrm{CBS}+/-1$ $\mathrm{C} 3 \mathrm{H}$ mice. Blood pressure measurement, western blotting (Caspase-9 and Caspase-3), q-PCR (Bax, Bcl-2), immunohistochemistry (cleaved Caspase-3) and TUNEL assay were used in this study. Blood pressure values were up-regulated in CBS+/- mice (diastolic: $118.4 \pm 9.8 \mathrm{mmHg}$; systolic: $153.9 \pm 12.7 \mathrm{mmHg}$; mean: $130 \pm 10 \mathrm{mmHg}$ ) compared to WT mice (diastolic: $101 \pm 15 \mathrm{mmHg}$; systolic: $139 \pm 10 \mathrm{mmHg}$; mean: $113 \pm 14 \mathrm{mmHg}$ ). q-PCR showed up-regulation of Bax mRNA expression in the mesenteric artery of $\mathrm{CBS}+/$ - mice compared to control. Western Blotting validated increase of Caspase- 9 and Caspase- 3 protein expressions in the mesenteric artery of CBS+/- mice compared to WT mice. In conclusion, our data suggested that HHcy-induced mitochondrial fission promotes Bax activation followed by mitochondrial apoptosis with activation of downstream proteases (Caspase-9, Caspase-3), leading to endothelial cell loss and arterial remodeling that contributes to hypertension.

\section{5}

\section{OVEREXPRESSION OF KV4.3 IN THE RVLM OF RATS WITH HEART FAILURE ATTENUATES SYMPATHETIC TONE}

BK Becker, H-J Wang, IH Zucker

University of Nebraska Medical Center, Omaha, Nebraska, USA

Chronic heart failure (CHF) is characterized by increased sympathetic tone, which contributes to the progression of cardiovascular damage. Our laboratory has previously observed reduced $\mathrm{Kv} 4.3$ expression in a key brainstem area that regulates sympathetic activity, the rostral ventrolateral medulla (RVLM), in rats with CHF. Reduced expression of Kv4.3 leads to increased neuronal excitability; however, the physiological importance of Kv4.3 in the RVLM has not yet been investigated during CHF. We hypothesized that overexpression of $\mathrm{Kv} 4.3$ in the RVLM of rats with CHF would reduce sympathetic tone. Sprague-Dawley rats with CHF were implanted with radiotelemetry and bilaterally injected with adenoviral-associated Kv4.3 (adKv4.3; $\mathrm{n}=6$ ) or GFP ( $\mathrm{d} G F P ; \mathrm{n}=5$ ) into the RVLM. Telemetry recordings were collected for 14 days post injection followed by terminal/anesthetized baroreflex RSNA experiments. Conscious heart rate was lower $(315.74 \pm 8.88$ vs. $362.84 \pm 5.81 \mathrm{bpm} ; \mathrm{p}<0.05)$, and $\mathrm{SDNN}$ was higher $(11.18 \pm 0.77$ vs. $8.61 \pm 0.63 \mathrm{~ms} ; \mathrm{p}<0.05)$ in adKv4.3 vs. GFP. Baseline RSNA was lower $(22.8 \pm 2.4$ vs. $42.4 \pm 5.7 \%$ of $\max ; \mathrm{p}<0.05)$, and the baroreflex sensitivity was higher $(2.84 \pm 0.64$ vs. $1.54 \pm 0.30$ Gmax; $\mathrm{p}<0.05)$ in adKv4.3 vs. adGFP. These data support the hypothesis that overexpression of $\mathrm{Kv} 4.3$ in the RVLM reduces sympathetic tone during CHF.

\section{6}

THE PROTECTIVE ROLE OF SARCOLEMMAL MEMBRANE ASSOCIATED PROTEIN ISOFORM 3 (SLMAP3) IN CARDIAC REMODELLING POST MI

\section{W Lefnaier, M Salih, BS Tuana}

\section{Faculty of Medicine, University of Ottawa, Ottawa, Ontario}

The Sarcolemmal Membrane Associated Protein 3 (SLMAP3) is a tailanchored membrane protein, which is ubiquitously expressed in tissues including myocardium. It is a component of subcellular membranes and the centrosome and appears to serves distinct role in cell growth and membrane biology (2). Recently, mutations in SLMAP3 isoform have been linked to Brugada syndrome which usually presents with aberrant electrical activity leading to cardiac dysfunction and death (1). Here, we have examined the effects of overexpressing SLMAP3 on postnatal heart function, pre and post myocardial infarction (MI). Transgenic ( $\mathrm{tg}$ ) mice with cardiac specific overexpression of SLMAP-3 were generated and assessed with echocardiography to measure the functional parameters, and western blotting to analyze the protein expression. The echocardiography of 8 week old tg mice showed $\sim 55 \%$ ejection fraction (EF) and $\sim 25 \%$ Fractional shortening (FS) which is equal to those of wild type mice $(n=30, P<0.05)$. MI was induced by LAD ligation ( 9 wk old mice) and echocardiography (at 1 wk post MI) showed a greater reduction of $\mathrm{EF}$ and $\mathrm{FS}$ in the wild type mice ( $\sim 37 \%$ and $\sim 17 \%$ ) compared to SLMAP3 tg mice $(\sim 45 \%$ and $\sim 22 \%$ ), respectively ( $\mathrm{n}=10$, $\mathrm{P}<0.05)$. Furthermore, Western blot analysis of apoptotic proteins such as Caspase- 3 and Bax was attenuated in myocardium from tg mice compared to wild type $(\mathrm{n}=3$ in each group with $\mathrm{MI}, \mathrm{P}<0.05)$. These data indicate that SLMAP3 levels may serve to limit cell death during cardiac remodeling post $\mathrm{MI}$ and protect the myocardium.

REFERENCES:

1. Ishikawa et al (2012). Circ Arrhym Electro 5:1098-1107.

2. Guzzo et al (2004). Biochem J. 381 (Pt 3):599-608.

\section{7}

\section{IMPACT OF HYDROGEN SULFIDE ON THE EPIGENETICS OF DIABETIC CARDIOMYOPATHY}

\section{$\underline{\text { S Veeranki, S Kundu, SC Tyagi }}$}

Department of Physiology and Biophysics, University of Louisville, Louisville, Kentucky, USA

Although Diabetic cardiomyopathy results in enhanced risk for heart failure, epigenetic changes leading to diabetic heart failure are unclear. Hydrogen sulfide $\left(\mathrm{H}_{2} \mathrm{~S}\right)$ has been implicated in the preservation of heart function owing to its anti-inflammatory and positive metabolic changes. In the current study, we investigated whether or not chronic H2S treatment (by giving NaHS) reverses diabetic cardiomyopathy using mouse model of type-1 diabetes: Akita mice. Regulators of mitochondrial biogenesis, calcium handling and molecules that regulate post-ischemic recovery were assayed by western blotting and Q-PCR. Further, we considered epigenetic modifications such as microRNA expression changes and DNA methylation alterations to understand the causes of diabetic heart failure with and without NaHS treatment for 30 days. Our data indicated that chronic H2S treatment significantly reduced the mitochondrial fission inducers: Drp-1 (24\%) and Fis-1 (17\%) in the Akita mouse hearts. Further, there was enhancement (10\%) in the SERCA2a expression after NaHS treatment in the diabetic hearts. Also, there was significant decrease (16\%) in TNF-1 $\alpha$ protein expression in diabetic hearts after NaHS treatment. In addition, there was significantly increased expression of post-ischemic recovery regulators such as: Notch3 (157\%), C-JUN (160\%), PGC-1 $\alpha$ (173\%), HIF- $2 \alpha$ (72\%), and NRF-1 (149\%) after NaHS treatment. These results suggest that the chronic NaHS treatment ameliorates diabetic cardiomyopathy through decreasing mitochondrial fission and inflammation and enhancing $\mathrm{Ca} 2+$ handling; as well as mitigating epigenetic changes leading to enhanced post-ischemic recovery potential.

Acknowledgement: Part of this study was supported by the NIH grants: HL-74185, NS-84823 and HL-108621.

\section{Session 2.5: Cellular and Molecular Aspects of Cardiovascular Diseases}

\section{2}

\section{CARDIAC REJUVENATION: A NEW FRONTIER IN THE MANAGEMENT OF HEART FAILURE \\ R-K Li}

Toronto General Research Institute, University Health Network, Department of Surgery, Division of Cardiovascular Surgery, University of Toronto, Toronto, Ontario

Heart failure results from cardiomyocyte necrosis after myocardial infarction. After infarction, the heart achieves limited repair through tissue resident and circulating stem cells. Resident cardiac stem cells are believed to be a legacy of cardiac development, residing in the myocardium but retaining the capacity for regeneration in times of injury. We recently found inceptive resident cardiac stem cells of hematopoietic origin that can independently direct endogenous repair. Age negatively influences the cardiac environment in transplantation recipients and reduces the cardiac tissue's functional capacity for effective stem cell transplantation. Whereas the cardiac environment in a young recipient can recuperate aged stem cells, young stem cells perform inadequately in aged recipients. This paradox suggests that successful stem cell implantation for cardiac repair depends not only on the regenerative 
capacity of the stem cells, but also on the proper myocardial milieu. We demonstrated recently that resident cardiac stem cells of hematopoietic origin govern cardiac repair. Rejuvenation of aged bone marrow increases regenerative capacity by restoring this resident cardiac stem cell niche. Cardiac resident stem cells play an important role in restoration of cardiac function and repair.

\section{8}

SCLERAXIS: A NOVEL TRANSCRIPTIONAL REGULATOR OF CARDIAC FIBROBLAST PHENOTYPE AND EXTRACELLULAR MATRIX PRODUCTION

\section{MP Czubryt, RA Bagchi, PL Roche}

Institute of Cardiovascular Sciences, St Boniface Hospital Research Centre and Department of Physiology and Pathophysiology, University of Manitoba, Winnipeg, Manitoba

The cardiac extracellular matrix (ECM) is a proteinaceous structure that both contributes to the physical structure of the heart and also serves as a source of physical and growth factor signals indicative of myocardial health and integrity. Resident cardiac fibroblasts respond to these signals, and are primarily responsible for ECM synthesis in the healthy heart. Cardiac stress including infarction, pressure overload and diabetes induces phenotype conversion of fibroblasts to myofibroblasts, which accelerate ECM production to cause fibrosis, in turn impairing cardiac function and rhythm. We have identified the transcription factor scleraxis as a novel regulator of cardiac fibroblast phenotype and ECM production. Scleraxis induces gene expression changes in cardiac fibroblasts indicative of activation to myofibroblasts, including increased expression of ECM proteins, direct transactivation of the $\alpha$-smooth muscle actin gene resulting in increased contractility, and induction of mesenchymal marker genes. Conversely, scleraxis knockdown reverses these effects and attenuates TGF $\beta / S m a d 3$ signaling, demonstrating that scleraxis is required for fibroblast-to-myofibroblast conversion. Scleraxis gene deletion in mice results in a $50 \%$ loss of cardiac fibroblasts and ECM, likely due in part to failure of fibroblast epithelioid precursors to undergo mesenchymal transition. Our data implicate scleraxis in the pathological events accompanying cardiac fibrosis. Supported by the Canadian Institutes of Health Research (grant MOP-136862).

\section{9}

\section{INCREASED M2 MACROPHAGE DIFFERENTIATION} REDUCES INFLAMMATION AND PLAQUE FORMATION IN APO E-/- MICE

\section{Singla}

University of Central Florida, Department of Molecular Biology and Microbiology, Burnett School of Biomedical Sciences, College of Medicine, Orlando, Florida, USA

Inflammation plays a significant role in the inception and development of atherosclerosis (ATH). Mechanisms of inflammation include the infiltration of monocytes into the injured area and subsequent differentiation into either pro-inflammatory M1 macrophages or anti-inflammatory M2 macrophages. We have reported that bone morphogenetic protein-7 (BMP-7) enhances M2 macrophage differentiation and anti-inflammatory cytokine secretion in cell culture model. However, whether BMP-7 would inhibit plaque formation in an animal model of ATH through increased M2 macrophage differentiation remains unknown. ATH was generated in male and female Apo E-/- mice via partial left carotid artery (PLCA) ligation and mice were divided into 3 groups: Sham, PLCA, and PLCA+BMP-7 (200ug/kg; i.v.). We performed histological, biochemical and RT-PCR techniques to evaluate macrophage polarization and their impact on the released pro- and anti-inflammatory cytokines. Our data shows that there was an inhibition of monocyte infiltration in the atherosclerotic area, and a decrease is associated with reduced pro-inflammatory cytokines (MCP-1, TNF- $\alpha$, and IL-6) following BMP-7 treatment. Moreover, our data suggest a significant $(\mathrm{p}<0.05)$ increase in M2 macrophage populations with consequential enhanced anti-inflammatory cytokine (IL-1RA, IL-10, and Arginase 1) expression following BMP-7 treatment. Formed plaque area in BMP-7 animals was significantly reduced and blood velocity functions were improved. In conclusion, we report that
BMP-7 has the potential to mediate cellular plasticity and mitigate the inflammatory immune response, which results in decreased plaque formation and improved blood velocity.

\section{Session 2.6: Diabetic Heart Failure}

\section{2}

\section{MYOCARDIAL INFARCTION ALTERS RENAL FUNCTION AND MORPHOLOGY}

LL Mehta, J Lu, X Wang

University of Arkansas for Medical Sciences and VAMC, Little Rock, Arkansas, USA

It is assumed, but not proven, that acute myocardial infarction affects function of remote organs- such as kidneys and brain. We examined renal function and morphology in mice subjected to left coronary artery (LCA) ligation. Wild-type (WT) mice were subjected to permanent LCA ligation resulting in extensive myocardial infarction. Soon after LCA ligation, there was a marked in rise in pro-inflammatory cytokines and MDA (index of oxidative stress) levels in circulation. On Day 3, renal function had markedly declined in association with swelling of gomeruli and tubules and mild fibrosis $(\mathrm{P}<0.05)$. There was a significant increase in the expression of LOX-1, IL-1 $\beta$ and VCAM-1, TBARS in the kidney. On Day 21, renal function showed some recovery, but there was progressive fibrosis in the kidneys. To determine the role of LOX-1 (a receptor for ox-LDL) overexpression in renal dysfunction following LCA occlusion, LOX-1 knockout mice subjected to total LCA ligation; these mice showed much less increase in systemic and renal pro-inflammatory cytokines and MDA levels, and much less structural alterations and renal functional decline than the WT mice (all $\mathrm{P}<0.05$ ). Cardiac function and survival rates were also better in the LOX-1 KO mice than in the WT mice $(\mathrm{P}<0.05)$. This study shows that acute myocardial ischemia results in renal dysfunction and histologic abnormalities reminiscent of acute renal injury. The morphologic changes continue to progress long after the acute myocardial ischemic event. This study also suggests that LOX-1 is a key modulator among multiple mechanisms underlying renal dysfunction following extensive myocardial infarction.

\section{3}

\section{RELEVANCE OF PERSONALIZED MEDICINE IN THE MANAGEMENT OF HEART FAILURE}

\section{RK Goyal, S Apparsundaram}

V ClinBio Labs, Sri Ramachandra University, Chennai, India;

V ClinBio LLC, Newark, New Jersey, USA

Heart failure remains one of the leading causes of death worldwide and therefore, it is a main focus of research and treatment. In spite of several developments with respect to the medications, the management of heart failure is still challenging for the physician. One major concern is the report that if drugs are not accounted for inter-individual differences, they may be either 'ineffective' or 'not completely effective' in 30-60\% of patients. The use of personalized medicine may allow the physician to provide a better therapy for patients in terms of efficacy, safety and treatment length, as well as to reduce the associated costs for the community. With the advent of state-of-the-art molecular profiling establishing clinical phenotype there is increasing trend to have profiling of markers to determine not only the prognosis but also for the management. There has been a remarkable growth in scientific publication on personalized medicine within the past few years in the cardiovascular field. However, application of personalized medicine into clinical treatment has been very slow. In the USA, $10 \%$ of Food and Drug Administration approved drugs include pharmacogenomic information on their labels and genetic testing is recommended or required for at least 12 FDA approved drugs. In developing countries like India as such data are scanty and there is no move for such recommendations by the drug authorities. We have undertaken studies to develop pharmacogenomics panel for the cardiac patients for the optimization of drug therapy based on genetic information for the management of heart failure. 


\section{4}

AMP-ACTIVATED PROTEIN KINASE AS A THERAPEUTIC TARGET FOR VASCULAR DISEASES

\section{M-H Zou}

Center of Molecular and Translational Medicine, Georgia State University, Atlanta, Georgia, USA

AMP-activated protein kinase (AMPK) is widely recognized as a sensor of energy status and redox status. As a result, overwhelming evidence supports that AMPK functions as a master regulator of cellular energy metabolism and redox homeostasis. Evidence accumulating from cell culture and animal experimentation has indicated that AMPK plays a critical role in the regulation of various vascular physiology, including vascular tone, as well as pathology processes, such as endothelial dysfunction and inflammation, as well as vascular smooth muscle cells phenotypic switching, proliferation/migration and calcification. AMPK activation also regulates the function of immune cells, especially macrophage, including proliferation, differentiation, fatty acid metabolism and cholesterol efflux. Moreover, clinical and laboratory studies show that pharmacological AMPK activators markedly prevent atherosclerosis development, high blood pressure, and diabetes complications. The absence or blockade of AMPK greatly accelerates the formation of atherosclerotic lesions, neointima and hypertension in animal experiments, suggesting that AMPK as a novel therapeutic target in the treatment of vascular diseases. Therefore, my talk will summarize how AMPK regulates vascular functions and how dysfunctional AMPK results in vascular diseases.

Session 2.7: Neuromodulation for the Treatment of Cardiovascular Disease

\section{5}

\section{AUTONOMIC REGULATION THERAPY IN HEART FAILURE JL Ardell}

UCLA Neurocardiology Research Center of Excellence and UCLA Arrhtyhmia Center, Los Angeles, California, USA

The cardiac nervous system, composed of the intracardiac ganglia, intrathoracic extracardiac ganglia, spinal cord, brainstem, and higher centers, coordinates regional cardiac function on a beat-to-beat basis. Globally, the cardiac nervous system is optimized to handle physiological stressors (e.g. orthostatic changes, exercise). However, it has not evolved a mechanism to adequately deal with catastrophic events such as myocardial infarction and the longerterm evolution of congestive heart failure. Imbalances within this neural network lead to excessive and stochastic activity, and underlie the mechanisms responsible for arrhythmias and heart failure. Autonomic Regulation Therapy (ART) is a rapidly emerging therapy in the management of congestive heart failure. Modulation of the cardiac neuronal hierarchy can be achieved with bioelectronic modulation of the spinal cord, cervical vagus, baroreceptors, or renal nerve ablation. With appropriate neuromodulation therapy, myocytes are rendered stress resistance, autonomic responsiveness for control of the heart is preserved, and the potential for fatal arrhythmias is reduced. Innovations in the field of Neurocardiology requires evolving new interfaces and analytics to evaluate neural network activity and cardiac electrical and mechanical function concurrently in normal and disease states. Understanding mechanistically what is being stimulated within the autonomic nervous system by such device-based therapy and how the system reacts to such stimuli is essential for optimizing stimulation parameters and for the future development of closed-loop ART.

\section{6}

\section{CARDIAC SYMPATHETIC AFFERENT DENERVATION: A NEW POTENTIAL THERAPY FOR HEART FAILURE}

H Wang

Dept of Cellular \& Integrative Physiology, University of Nebraska

Medical Center, Omaha, Nebraska, USA

The chronic heart failure state (CHF) is characterized by neurohumoral activation and cardiac remodeling (fibrosis, dilation and hypertrophy). In the CHF state a potent cardiogenic reflex known as the "cardiac sympathetic afferent reflex" (CSAR) is activated by an increase in the activity of sympathetic afferents on or near the surface of the ventricles. This excitatory reflex, in part, drives sympathetic efferent nerve activity to the heart and the periphery. Recent data from our lab demonstrated that selective removal of these afferents using the TRPV1 receptor agonist resiniferitoxn (RTX), at the time of myocardial infarction (MI) in rats, resulted in a decrease in cardiac and renal sympathetic nerve activity and norepinephrine excretion 9-11 weeks following the MI. In addition to its classical sympatho-excitatory effect, we also found that these cardiac afferents are pro-inflammatory and can aggravate local cardiac extracellular matrix (ECM) remodeling post $\mathrm{MI}$ by activation of matrix metalloproteinase (MMP) activity, which can be largely prevented by RTX treatment. This intervention also decreased the remodeling process by reducing cardiac fibrosis. The resultant effect of RTX treatment was a decrease in LV end diastolic pressure and an increase in diastolic function along with an increase in the cardiac response to isoproterenol (cardiac reserve). In addition, we developed a clinically applicable delivery route (epidural T1-T4 peri-ganglion administration) targeting RTX to the afferent neuronal soma in the dorsal root ganglia. These data suggest that RTX therapy may have clinical relevance for improving cardiac and autonomic dysfunction in CHF.

\section{7}

\section{DEVICE-BASED NEUROMODULATION FOR HYPERTENSION THERAPY: MECHANISMS AND THERAPEUTIC IMPLICATIONS}

\section{TE Lohmeier}

The University of Mississippi Medical Center, Jackson Mississippi, USA Recent technical advances have stimulated interest in device-based therapy for the treatment of resistant hypertension defined as blood pressure that remains above goal in spite of the concurrent use of $\geq 3$ antihypertensive agents, with one being a diuretic and all given at optimized doses. Electrical activation of the carotid baroreflex (BA) and percutaneous renal nerve ablation provide global- and renal-specific suppression of sympathetic activity, respectively, and are currently being evaluated in clinical trials for the treatment of resistant hypertension. While clinical trials show that BA and renal denervation diminish the severity of hypertension in many with resistant hypertension, a reduction in arterial pressure does not always occur, and the conditions for a favorable response are largely unknown. A recent report indicating that renal sympathetic nerve activity (RSNA) is elevated in many patients with resistant hypertension is consistent with the hypothesis that the renal nerves likely represent the critical link between increased central sympathetic outflow and impaired renal function that sustains resistant hypertension, and that both devices may lower arterial pressure by reducing the intensity of RSNA. However, the relationship between basal RSNA and the subsequent arterial pressure response to BA and renal denervation is unclear, as are the conditions that modify a direct relationship if one were to exist. Experimental studies provide insight into the mechanisms that account for chronic lowering of arterial pressure during suppression of sympathetic activity by BA and renal denervation and may help identify the subsets of this heterogeneous hypertensive population who stand to benefit the most from device-based therapy.

\section{8}

SYMPATHO-EXCITATORY REFLEXES IN CARDIOVASCULAR DISEASE: THE EFFICACY IN RESTRAINING THE CAROTID BODY

\section{HD Schultz}

Department of Cellular and Integrative Physiology, University of Nebraska Medical Center, Omaha, Nebraska, USA

The treatment of chronic heart failure (CHF) remains an important focus for new and more effective clinical strategies. In CHF, sympathetic overactivity plays an important role in the development and progression of the cardiac and renal dysfunction (cardio-renal syndrome), and is often associated with breathing dysregulation, which in turn aggravates the autonomic imbalance. Evidence demonstrates that the elevations in sympathetic activity and breathing instability in CHF are driven, at least in part, by 
maladaptive activation of the carotid body $(\mathrm{CB})$ chemoreflex driven by a tonic increase in $\mathrm{CB}$ afferent nerve activity. Discussion will focus on mechanisms that alter $\mathrm{CB}$ afferent activity in $\mathrm{CHF}$ and its consequence on reflex control of autonomic, respiratory, renal, and cardiac function in animal models of CHF. The potential translational impact of targeting the $\mathrm{CB}$ in the treatment of cardio-renal syndrome in patients with $\mathrm{CHF}$ and its relevance to other cardio-respiratory diseases will be discussed.

Session 2.8: Dr Bruce McManus Young Investigator Presentations

\section{9}

\section{PTEN INHIBITOR REDUCES CARDIAC REMODELING IN DOXORUBICIN-INDUCED CARDIOMYOPATHY}

\section{TA Johnson, DK Singla}

Burnett School of Biomedical Sciences, College of Medicine, University of Central Florida, Orlando, Florida, USA

BACKGROUND: Doxorubicin (Doxo) is one of multiple anthracycline drugs used to effectively treat various forms of cancer. Unfortunately, Doxo treatment stimulates adverse cardiac remodeling and subsequent heart failure. We have previously demonstrated that transplanted embryonic stem (ES) cells and their conditioned medium (CM) modulate the PTEN pathway and reduce apoptosis, fibrosis and hypertrophy in a Doxo-Induced Cardiomyopathy (DIC) model. VOOHpic (VO), the most potent known PTEN inhibitor, has shown to be cardioprotective against ischemia-reperfusion injury; however VO efficacy in a DIC model has not been explored. HYPOTHESIS: Intraperitoneal (IP) delivery of VO blunts PTEN expression and protects the heart from doxorubicin-induced cardiac remodeling. METHODS: Animals were divided into three groups; Group 1: Control (Saline), Group 2: Doxo (12 mg/kg, Cumulative dose) and Group 3: Doxo and VO $(12 \mathrm{mg} / \mathrm{kg}$ and $10 \mu \mathrm{g} / \mathrm{kg}$ cumulative doses $)$ via IP injection. One week post-DIC, mice were subjected to echocardiography to examine cardiac function, sacrificed and hearts were harvested for further analysis.

RESULTS: Immunohistochemistry staining revealed a significant $(\mathrm{p}<0.05)$ decrease in apoptotic cardiomyocytes in Doxo-VO treated hearts compared with Doxo. Furthermore, Hematoxylin and Eosin (H\&E) and Masson's Trichrome histological stains confirmed reduced hypertrophy, interstitial and vascular fibrosis in Doxo-VO treated subjects compared to Doxo group. Western Blotting confirmed the reduction in PTEN levels in Doxo-VO subjects compared to Doxo hearts. Heart function was significantly improved upon Doxo-VO treatment compared to Doxo group. CONCLUSION: Our data suggest that $\mathrm{VO}$ treatment attenuates adverse cardiac remodeling and improves heart function in the DIC heart.

\section{0}

\section{MIR-133A REGULATES CARDIAC AUTOPHAGY IN DIABETICS}

SS Nandi ${ }^{1}$, PK Mishra ${ }^{1,2}$

${ }^{1}$ Department of Cellular and Integrative Physiology; ${ }^{2}$ Department of Anesthesiology, University of Nebraska Medical Center, Omaha, Nebraska, USA

Attenuated miR-133a is associated with induced autophagy in diabetic heart failure patients. However, the underlying molecular mechanism is unclear. We hypothesized that diabetes-mediated downregulation of miR133a induces autophagy, whereas overexpression of miR-133a in diabetic hearts would restore the basal level of autophagy. To test this hypothesis, we used four in vivo groups: C57BL/6J (WT), Ins2+/-Akita (T1D), and Akita treated with miR-133a mimic $(\mathrm{Ak}+\mathrm{miR})$ or scramble miRNA $(\mathrm{Ak}+\mathrm{scm})$; and four in vitro treatment groups of HL1-cardiomyocytes: low (LG-5mM), high (HG-25mM) doses of D-glucose, $\mathrm{HG}+\mathrm{miR}$ and $\mathrm{HG}+\mathrm{scm}$. We performed transmission electron microscopy (TEM) and several molecular assays to examine autophagosome formation, degradation, and autophagic flux in the heart. Our results showed increased protein level of autophagosome formation marker LC3B $(1.91 \pm 0.01)$ in Akita and its downregulation $(0.84 \pm 0.04)$ in $A k+m i R$ as fold change compared to the WT heart. Further,
TEM analyses revealed an increased number of autophagosomes/total area in Akita as compared to the WT heart but it was decreased in Ak+miR. In vitro studies showed increased autophagosome formation in HG (LC3B: $1.44 \pm 0.02)$ and its downregulation $(0.76 \pm 0.02)$ in $\mathrm{HG}+\mathrm{miR}$ as fold change of LG. Further, LAMP2 (involved in autophagosome degradation) was upregulated in $\mathrm{HG}(1.37 \pm 0.1)$ and restored $(1.09 \pm 0.15)$ in $\mathrm{HG}+\mathrm{miR}$ as fold change compared to LG. The mCherry-EGFP-LC3B plasmid-based analyses elicited increased autophagic flux in HG $(5.58 \pm 2.26)$ as fold change compared to $\mathrm{LG}$, and its restoration in $\mathrm{HG}+\mathrm{miR}(0.90 \pm 0.9)$. Additionally, luciferase reporter assay revealed that miR-133a targets LC3B. In conclusion, miR-133a regulates autophagosome formation, degradation and autophagic flux in diabetic hearts.

Acknowledgements: NIH grants: HL113281 and HL116205

\section{1}

\section{IMPROVED SURVIVAL AND CARDIAC FUNCTION FOLLOWING MYOCARDIAL INFARCTION IN MOUSE MAST CELL PROTEASE-4 DEFICIENT MICE}

M Houde $^{1}$, M Lepage ${ }^{1}$, R Lecomte $^{1}$, A Tremblay ${ }^{1}$, L Tremblay ${ }^{1}$, AG Schwertani $^{2}$, P D'Orléans-Juste ${ }^{1}$

${ }^{1}$ Institute of Pharmacology, Université de Sherbrooke, Sherbrooke; ${ }^{2}$ Division of Cardiology, McGill University, Montreal, Quebec

Albeit treatment of myocardial infarction with angiotensin converting enzyme inhibitors improves prognostic, circulating angiotensin-II levels rapidly return to baseline values. Chymase, which is released from mast cells in heart failure, is an important cardiac Ang-II producing enzyme. We aimed to assess the impact of the deletion of a chymase isoform, mouse mast cell protease-4 (mMCP-4), in the progress of irreversible myocardial infarction. Anaesthetised mMCP-4 KO mice and C57BL6/J wild-type (WT) congeners were intubated and the left anterior descending (LAD) coronary arteries ligated. Mice were left to recover for 72 hours or 28 days before euthanasia. At 72 hours, some mice were subjected to cardiac positron emission tomography (cPET) to assess left ventricular function, after which mice were sacrificed and hearts collected for histology and biochemical measurements.

Genetic deletion of mMCP-4 improved 28-day survival compared to WT mice ( 93.3 vs $40.0 \%, \mathrm{p}<0.001)$. 72 -hour cPET shows that infarct size and end-diastolic volume are lower, while ejection fraction is higher, in mMCP-4 KO mice when compared to WT animals $(\mathrm{p}<0.02)$.

The deletion of mMCP-4 protects left ventricular functional integrity post-infarction, resulting in an improved survival.

This work was supported by the Canadian Institutes for Health Research (CIHR).

\section{2}

\section{INCREASED MITOCHONDRIAL SUPEROXIDE IN THE BRAIN, BUT NOT PERIPHERY, SENSITIZES MICE TO ANGIOTENSIN II-MEDIATED HYPERTENSION}

\section{A] Case, J Tian, MC Zimmerman}

\section{University of Nebraska Medical Center, Omaha, Nebraska, USA}

Mitochondrial superoxide (O2•-) is essential for angiotensin II (AngII)dependent hypertension. However, it remains unknown if increased mitochondrial $\mathrm{O} 2 \bullet-$ flux is sufficient to affect hemodynamics. We hypothesized that elevated levels of systemic mitochondrial O2 - - exacerbates AngIIinduced hypertension. To test this, we utilized a conditional mouse model of manganese superoxide dismutase knock-out (MnSODlox/lox). Using a systemic inducible promoter to drive cre-recombinase expression, MnSOD was knocked-down $(30-98 \%, \mathrm{p}<0.05)$ in peripheral organs, but not in the brain. Interestingly, mean arterial pressure (MAP) and heart rate were not statistically different in MnSOD knock-downs versus controls \pm subcutaneous AngII (400 ng/ $\mathrm{kg} / \mathrm{min}$ ) infusion. Due to these unexpected results, we examined the role of elevated mitochondrial $\mathrm{O}_{2} \bullet-$ levels specifically in the subfornical organ (SFO) in the brain. After targeting cre-recombinase to this region, we observed a $60 \%$ decrease of $\mathrm{MnSOD}(\mathrm{p}<0.05)$ with concomitant increase in mitochondrial $\mathrm{O} 2 \bullet-$ in the SFO. Intriguingly, while these mice demonstrated no change in baseline MAP $(92.8 \pm 0.4 \mathrm{mmHg}$ vs. $93.1 \pm 0.4 \mathrm{mmHg}$ - knock-down vs. control), they displayed a significant elevation in MAP upon peripheral AngII infusion (MAPmax $=137.8 \pm 2.7$ 
mmHg vs. $128.3 \pm 3.3 \mathrm{mmHg}$ - knock-down vs. control, $\mathrm{p}<0.05)$. Taken together, our data suggest that dysregulation of mitochondrial redox status in the SFO may be a predisposition to increased responsiveness to hypertensive stimuli, such as AngII.

This work was supported by NIH R01HL103942 and NIH F32HL122021.

\section{3}

THE CARDIAC SPECIFIC ISOFORM OF THE TAIL-ANCHORED MEMBRANE PROTEIN SLMAP1 ENHANCES GLUT4 LEVELS THROUGH A MECHANISM INVOLVING THE SNARE COMPLEX AND SIZE EXPANSION OF EARLY ENDOSOMES

T Rehmani $^{1}$, A Dewan ${ }^{1}$, M Salih ${ }^{1}$, C Triggle ${ }^{2}$, H Ding ${ }^{2}$, BS Tuana ${ }^{1}$ ${ }^{1}$ Faculty of Medicine, University of Ottawa, Ottawa, Ontario; ${ }^{2}$ Weill Cornell Medical College, Doha, Qatar

The tail anchored Sarcolemmal Membrane Associated Protein isoform 1 (SLMAP1) is structurally similar to the super family of membrane proteins involved in intracellular vesicle transport and fusion. Enhanced levels of SLMAP1 increased glucose uptake and metabolism by upregulating GLUT 4 levels in cardiomyocytes [1]. A significant size expansion of early endosomes ( 43 fold \pm 5 ) was evident in cardiomyocytes isolated from transgenic (tg) myocardium expressing SLMAP 1. GLUT4 mRNA was unaltered by SLMAP1 expression. In order to test the hypothesis that increased GLUT4 protein levels were due to enhanced composition or fusion of early endosomes we examined the components involved in early endosome formation. In particular the expression and localization of SNARE proteins SNAP23 and Syntaxin-4 which enable fusion of early endosome membrane vesicles were examined. Western blot analysis revealed a significant up-regulation of these proteins SNAP23, $(187.94 \% \pm 60.28 \%, n=6$, $\mathrm{P}<0.05)$, Syntaxin $4(133 \% \pm 53.03 .4 \%, \mathrm{n}=5, \mathrm{P}<0.01)$ in myocardium from SLMAP1 tg mice compared with wild type litter mates. Immunofluorescence analysis indicated a significant co-localization of SLMAP1 with SNAP23 and Syntaxin 4 in enlarged membrane vesicles which were identified as early endosomes with the marker EEA1 in cardiomyocytes. These data indicate that SLMAP1 can specifically enhance GLUT4 protein levels by the expression of the SNARE complex in myocardium via a mechanism involving the fusion of early endosomes and their size expansion. The enlarged vesicles/vacuoles noted in SLMAP1 TG myocardium [2] in part represent the early endosomes. Thus SLMAP1 can uniquely regulate GLUT4 levels, glucose uptake and metabolism through a mechanism involving fusion and size expansion of early endosomes. Enhanced expression of SLMAP1 is being evaluated in targeting the diabetic state. REFERENCES:

1. Dewan et al (2013). Proceedings of the British Pharmacological Society $<$ http://www.pa2online.org/abstracts/voll 1issue3abst216p.pdf>

2. Nader M et al (2012). Am J Physio Heart Circ Physiol 302:1138-1145.

\section{Session 2.9: Eric Olson Young Faculty Competition (Biomedical Sciences)}

\section{4}

\section{UPREGULATION OF ANGIOTENSINOGEN (AGT) IN THE PARAVENTRICULAR NUCLEUS (PVN) OF THE} HYPOTHALAMUS DURING CHRONIC HEART FAILURE (CHF): ROLE OF MIR-133A

NM Sharma, SS Nandi, X Liu, H Zheng, PK Mishra, KP Patel Department of Cellular \& Integrative Physiology, University of Nebraska Medical Center, Omaha, Nebraska, USA

Emerging evidence has demonstrated that activation of the renin-angiotensin system (RAS) is closely associated with enhance sympathetic activation in CHF. The contribution of changes in AGT per se remains to be examined. In this study, we observed a 12 -fold increase in levels of AGT gene in the PVN of CHF rats compared to sham-operated controls with a concomitant increase in Ang II type 1 receptors (AT1R). In order to elucidate the molecular mechanism of increased expression of AGT, we performed in silico analysis of 3'-UTR of AGT gene and found potential binding sites for microRNA (miR)-133a. Consistent with these observations, we found a 1.9-fold decrease in miR-133a expression in the PVN of rats with CHF. We hypothesized that decreased expression of miR-133a may be responsible for increased expression of AGT in the PVN. To test this hypothesis we used NG108 (neuroblastoma x glioma) cells, endogenously expressing RAS components, as our in vitro model. Overexpression of miR133a mimic (pEZX-MRO3-miR133a) resulted in 1.4 and 1.5-fold decrease in AGT and AT1R mRNA levels, respectively. Further, luciferase reporter assay confirmed 3.4-fold reduction in luciferase activity with miR133a mimic. Validating our in vitro data, preliminary studies revealed, mIR133a upregulation in the PVN of rats with CHF ameliorated the increased AGT expression $(0.44 \pm 0.05$ CHF-GFP vs. $0.29 \pm 0.03 *$ CHF-mIR-133a). These results suggest that AGT is targeted by miR-133a, revealing a novel and unique role for miR-133a in the regulation of Ang II within the PVN, which potentially contributes to sympathoexcitation during CHF. Supported by NIH-HL62222 and AHA-14SDG19980007 grants.

\section{5}

\section{EPIGENETIC MODIFICATION OF BRAIN (PRO)RENIN RECEPTOR CONTRIBUTES TO THE DEVELOPMENT OF DOCA-SALT HYPERTENSION VIA EPITHELIAL SODIUM CHANNEL}

\section{W Li, Y Feng}

Department of Pharmacology and Physiology \& Cell Biology, University of Nevada School of Medicine, Reno, Nevada, USA We recently reported that elevated brain (pro)renin receptor (PRR) level contributes to the development of DOCA-salt hypertension; however, the underlining mechanism remains unknown. To test our hypothesis that epigenetic mechanism is involved in the regulation of PRR during hypertension, C57Bl/6J mice were treated with SHAM or DOCA-salt for 3 weeks. We found an increase (fold change) in histone 3 lysine 4 trimethylation $(\mathrm{H} 3 \mathrm{~K} 4 \mathrm{me} 3)$ on the PRR promoter in DOCA-salt $(2.45 \pm 0.36) \mathrm{com}$ pared with SHAM treated mice $(1.00 \pm 0.16, \quad \mathrm{P}<0.05)$. H3K4 methyltransferases (HMT) activity (OD/hour/mg protein), an enzyme complex responsible for $\mathrm{H} 3 \mathrm{~K} 4$ methylation was increased after DOCA-salt treatment $(45 \pm 2, \mathrm{P}<0.05)$ compared with SHAM $(28 \pm 2)$. To examine the mechanisms of elevated HMT activity, we treated neurons with control (155mM sodium), high salt (170 mM sodium), aldosterone (100 nM), or high salt+aldosterone with or without epithelial sodium channel (ENaC) blocker (amiloride, $100 \mathrm{nM}$ ) for 2 hours and test the HMT activity and PRR expression levels. High salt+aldosterone increased HMT activity $(41 \pm 3$ vs. $24 \pm 2, P<0.05)$ and PRR mRNA $(1.6 \pm 0.1$ vs. $1.0 \pm 0.1$ fold change, $\mathrm{P}<0.05)$ levels compare to control. Amiloride attenuated HMT activity $(25 \pm 4.1, \mathrm{P}<0.05)$ and PRR mRNA levels $(0.98 \pm 0.1, \mathrm{P}<0.05)$ compared to high salt+aldosterone treatment. We conclude that $\mathrm{ENaC}$ may be responsible for epigenetic up-regulation of brain PRR and thus the increase in BP during DOCA-salt hypertension.

\section{6}

\section{ANGIOTENSIN TYPE 2 RECEPTOR KNOCKOUT MICE EXHIBIT BLUNTED ARTERIAL BAROREFLEX SENSITIVITY AND OVERACTIVATED SYMPATHETIC TONE}

J Gao ${ }^{1}$, J Chao ${ }^{1}$, T Walther ${ }^{2}$, F Gembardt ${ }^{2}$, IH Zucker ${ }^{1}, \underline{L}_{\text {Gao }}{ }^{1}$ ${ }^{1}$ Department of Cellular \& Integrative Physiology, University of Nebraska Medical Center, Omaha, Nebraska, USA; ${ }^{2}$ Centre for Cardiovascular and Metabolic Research, Hull York Medical School, University of Hull, Hull, United Kingdom

Angiotensin type 2 receptor (AT2R) knockout (KO) mice is characterized with a hypertension. The underlying mechanism(s) are not completely elucidated. Previous data from our laboratory demonstrated a sympathoinhibition by central AT2R stimulation. In this experiment, we evaluated the potential contribution of sympathetic activity to the hypertension of this transgenic mouse. We found that, compared to wild type (WT), (1) $\mathrm{KO}$ mice exhibited higher blood pressure (MAP) (KO 85.0 \pm 3.7 vs WT $74.7 \pm 3.8 \mathrm{mmHg}, \mathrm{p}<0.05)$ and increased renal sympathetic nerve activity (RSNA) (KO 31.3 \pm 4.8 vs WT $18.5 \pm 0.9 \% \max , \mathrm{p}<0.05$ ); (2) Baroreflex sensitivity was significantly blunted in $\mathrm{KO}$ mice (Gmax for $\mathrm{HR}$ : $\mathrm{KO} 2.7 \pm 1.1$ 
vs WT $8.4 \pm 2.7 \mathrm{bpm} / \mathrm{mmHg}$; $<<0.05$; Gmax for RSNA: KO $-1.2 \pm 1.7$ vs WT $12.9 \pm 1.3 \% \mathrm{max} / \mathrm{mmHg}$; $<<0.05$ ); (3) In $\mathrm{KO}$ mice, icv Ang II evoked bigger pressor response and sympatho-excitation, whereas icv losartan induced a bigger fall of MAP and RSNA; (4) in the nucleus tractus solitarius (NTS) of KO mice, the superoxide production (detected by DHE staining) and AT1R expression (measured by Western blot analysis) were significantly upregulated as compared with the WT mice. These results suggested that the increased sympathetic activity contributed to the hypertension in AT2R deficient mice. Our data also implied that the sympathoexcitation in this mouse model was due to the impaired baroreflex function and enhanced central AT1R signaling, in which the enhanced oxidative stress in the NTS was involved.

This project is supported by NIH R01HL093028 (Dr Lie Gao).

\section{7}

ABLATION OF COX-2 ATTENUATES ADIPOSE INFLAMMATION BUT IMPAIRS METABOLIC HOMEOSTASIS IN OBESITY

V Saraswathi, K Meena, C Perriotte-Olson, C Desouza, J Bowen, N Adi, R Ramalingam

Department of Internal Medicine/DEM, University of Nebraska

Medical Center, and VA Nebraska Western, lowa Health Care

System, Omaha, Nebraska, USA

Obesity predisposes individuals to type 2 diabetes and cardiovascular disease (CVD). The role

of cyclooxygenase-2 (COX-2), a well-known pharmaceutical target for its role in inflammation in regulating obesity and its co-morbidities, in particular, dyslipidemia, remains unclear. The objective of this study is to determine the role of COX-2 in modulating metabolic inflammation and systemic lipid homeostasis in obesity. Wild type (WT) and COX-2 knockout (COX-2-1-) mice were fed a high fat (HF, 45\% fat calories) diet for 13 wk. The expression of macrophage and inflammatory markers were reduced in visceral adipose tissue (VAT) and macrophageenriched VAT stromal cells collected from COX-2-1- mice compared to WT mice on a HF diet. However, VAT mass was significantly increased in COX-2 / - mice. Interestingly, the protein expression of ATG7, an autophagy marker, was significantly reduced in COX-2-1- mice. Of note, autophagy is evolving as a novel mechanism regulating lipid metabolism. As with VAT, the hepatic expression of macrophage and inflammatory markers were reduced with a concomitant reduction in autophagy markers in COX-2-1-mice. This was associated with an increase in plasma total cholesterol in COX-2-/- mice. Furthermore, the genes promoting hepatic cholesterol metabolism were down-regulated in COX-2-1- mice. Not only global deletion but also COX-2 deletion in hematopoietic cells resulted in a decrease in autophagy markers and an increase in adiposity. Our data suggest that although COX-2 inhibition can be effective in reducing inflammation, it may impair lipid homeostasis likely via diminished autophagy which, in turn, can increase the risk for CVD.

This work was supported, in part, by a Scientist Development Grant from the American Heart Association.

\section{Session 2.10: Exercise Training in Cardiovascular Disease}

\section{8}

\section{CENTRAL NEURAL CONTROL OF SYMPATHETIC NERVE ACTIVITY IN HEART FAILURE FOLLOWING EXERCISE TRAINING \\ H Zheng, KP Patel}

Department of Cellular and Integrative Physiology, University of Nebraska Medical Center, Omaha, Nebraska, USA

Typical characteristics of chronic congestive heart failure (HF) are increased sympathetic drive, altered autonomic reflexes, and altered body fluid regulation. These abnormalities lead to an increased risk of mortality, particularly in the late stage of chronic HF. Recent evidence suggests that central nervous system (CNS) mechanisms may be important in these abnormalities during HF. Exercise training (ExT) has emerged as a nonpharmacological therapeutic strategy substitute with significant benefit to patients with HF. The mechanism(s) by which ExT improves the clinical status of HF patients is not fully known. Our studies have provided evidence that ExT significantly alleviates the increased sympathetic drive, altered autonomic reflexes and altered body fluid regulation in HF. The studies have revealed that ExT improves the altered inhibitory pathway utilizing nitric oxide and GABA mechanisms within the paraventricular nucleus (PVN) in HF. Exercise training alleviates elevated sympathetic outflow in HF, through normalization of excitatory glutamatergic and angiotensinergic mechanisms within the PVN. Exercise training also improves volume reflex function and thus fluid balance in HF. We conclude that improvement of the enhanced CNS-mediated increase in sympathetic outflow, specifically to the kidneys related to fluid balance, contributes to the beneficial effects of ExT in HF.

\section{9 EXERCISE AND EXOSOME IN CARDIOVASCULAR HEALTH AND DISEASE}

$\underline{\text { SC Tyagi, M Phil }}$

Department of Physiology \& Biophysics, University of Louisville School of Medicine, Louisville, Kentucky, USA

Remodeling and myocardial matrix metabolism contributes to cardiac endothelium-myocyte (perivascular fibrosis), myocyte-myocyte (interstitial fibrosis), and mitochondrion-myocyte (fusion and fission) coupling. Matrix metalloproteinases (MMPs), and tissue inhibitor of metalloproteinases (TIMPs) play differential roles in different tissues and diseases. For example, although present in the heart, MMP-3 is known as stromelysin (i.e., stromal tissue enzyme). Interestingly, TIMP-3 causes apoptosis. Exercise and nutrition are synergistic in the mitigation of diseases: exercise releases exosomes containing miRNAs. Nutrition/vitamins B6 and B12 regulate the metabolism of homocysteine (an epigenetic byproduct of DNA/RNA/protein methylation). Thus, epigenetic silencing is an important therapeutic target. The statistical analysis of cohorts may be less indicative for the treatment of a disease, particularly if the 2 twins are different in terms of responding to the medicine for the same disease, therefore, personalized medicine is the future of therapy.

\section{6}

\section{CATIONIC PEPTIDES AND PROTEINS IN INFLAMMATION AND ATHEROSCLEROSIS: NOVEL INSIGHTS}

\section{S Parthasarathy}

The University of Central Florida, Orlando, Florida, USA

BACKGROUND: Apolipoprotein A1 and apolipoprotein E mimetic peptides have attracted attention due to their ability to reduce atherosclerosis and exhibit antioxidant, anti-inflammatory, and hypolipidemic properties. We tested whether non-lipoprotein related cationic peptides and proteins would have anti-inflammatory properties both in vitro and in vivo. We also tested whether ApoB100 of low density lipoprotein (N-LDL) would have similar properties.

METHODS: 5F-mimetic peptide, LL27 derived from the anti-microbial peptide CAMP, and a human glycodelin derived peptide (Gdn-P), were commercially synthesized. N-LDL was prepared and used. The ability of these peptides and protein to neutralize charges of modified lipoproteins, as well as attenuate macrophage uptake and inflammation, were analyzed. Oxidized LDL (Ox-LDL) was pretreated with increasing concentrations of peptides and $\mathrm{N}$-LDL to evaluate charge neutralizing properties of the peptides as well as that of the protein

(ApoB100). RAW cells were incubated with LPS or Ox-LDL pretreated with and without peptides and N-LDL. RNA was isolated from treated cells and real time PCR was performed using mouse IL- $1 \alpha$ and IL- 6 primers.

RESULTS: Cationic peptides as well as ApoB100 protein of N-LDL decomposed the peroxide content of 13-HPODE. Incubation of Ox-LDL and Ac-LDL with the peptides as well as ApoB100 resulted in charge neutralization as noted by agarose gel electrophoresis. Pre-incubation of the peptides with modified lipoproteins reduced the uptake of the latter by macrophages and foam cell formation as detected by Oil-Red $\mathrm{O}$ staining. 
Reduced inflammation was observed in the presence of N-LDL as compared to LPS/Ox-LDL.

CONCLUSIONS: Based on these studies, we postulate that cationic peptides and protein might have properties that would a) affect events that are unrelated to lipid lowering, b) might play an additional role in immune competent cells, including macrophages, and c) might interact with other biologically important anionic molecules, including lipids and proteins. We also predict that lysine-rich cationic peptides and proteins could have therapeutic potential in reducing CVD/atherosclerosis-associated inflammation.

\section{0}

\section{A RAT MODEL SYSTEM OF LOW AND HIGH SUSCEPTIBILITY TO DISEASE RISKS}

\section{SL Britton, LG Koch}

Department of Anesthesiology, University of Michigan, Ann Arbor, Michigan, USA

Large-scale clinical studies show that low exercise capacity is a stronger predictor of morbidity and mortality relative to other common risk factors including hypertension, type II diabetes, or smoking. This robust association led us to formulate that variation in capacity for energy transfer is a central mechanistic determinant of the divide between complex disease and health (Energy Transfer Hypothesis). As an unbiased test of this hypothesis we reasoned that, divergent artificial selection of rats based on low and high intrinsic treadmill running capacity would yield contrasting aerobic models that also divide for disease risks.

Artificial selection for intrinsic endurance running capacity was started in 1996 using genetically heterogeneous N: NIH stock of rats as a founder population $(n=168)$ to develop lines of low capacity runners (LCR) and high capacity runners (HCR). Selection for low and high capacity was based upon distance run to exhaustion on a motorized treadmill. By generation 35 of breeding (2014) the LCR rats exhausted on average after 205 $\mathrm{m}$ and the HCR exhausted after $2293 \mathrm{~m}$ of distance run. Studies have revealed that a surprisingly large number of clinically relevant disease risks segregated more strongly in the LCR relative to the HCR. That is, the LCR demonstrate reduced longevity, premature aging, decreases in phosphorylating respiration, and increased susceptibility for ventricular fibrillation, intra-cerebral hemorrhage, metabolic syndrome, fatty liver disease, disordered sleep, Alzheimer's neurodegeneration, pulmonary hypertension, and vulnerability to inducible cancer. Apparently disparate conditions segregated with selection for aerobic capacity suggesting we may discover new mechanistic commonalities underlying complex diseases.

\section{1}

\section{CLINICAL OUTCOMES AND THE CHALLENGE OF ADHERENCE TO EXERCISE IN HEART FAILURE}

\section{B Pozehl}

\section{University of Nebraska Medical Center-Lincoln Division, Lincoln,}

Nebraska, USA

Exercise training trials in patients with heart failure (HF) have demonstrated improvements in exercise capacity, functional disability, and clinical outcomes including quality of life. The majority of these trials have focused on patients with a diagnosis of HF with reduced ejection fraction (HFrEF with only a few focused on patients with HF with preserved ejection fraction (HFpEF). The multi-center HF-ACTION trial demonstrated the safety of exercise training and led to the eventual approval of the US Center for Medicare Services (CMS) for cardiac rehabilitation in HFrEF. The HF-ACTION trial had only approximately $35 \%$ of patients adhering to 120 minutes of aerobic exercise per week at the end of 18 months. Adherence to exercise is reported by patients to be more difficult than any of the other required behavioral changes for $\mathrm{HF}$ including diet, medications, smoking cessation or keeping appointments. Patients identify lack of motivation, lack of energy, and physical symptoms as primary reasons for not adhering to exercise. They report a lack of skills for exercise and fear of exercise with a "bad heart". System level factors also impact adherence to exercise with a significant under-referral to cardiac rehabilitation by providers and a general lack of emphasis on the benefits of exercise training as an adjunct to optimal pharmacologic therapy. Clearly there are many factors impacting adherence to exercise and as a result patients are not exercising at levels to achieve the demonstrated dose-response for benefits from exercise.

\section{Session 2.11: Epigenetics and Cardiovascular Disease}

\section{2}

SIRT3 DEACETYLASE IS A GUARDIAN OF MITOCHONDRIA, WHICH PROTECTS THE HEART FROM DESCENDING TO FAILURE

MP Gupta, V Pillai, S Samant

Center of Cardiac Cell Biology, Department of Surgery, Basic Science Division, University of Chicago, Chicago, Illinois, USA

SIRT3 belongs to the sirtuin family of $\mathrm{NAD}^{+}$-dependent deacetylases. Members of this family are emerging as key regulators of many biological functions, including cell growth, apoptosis, metabolism and longevity. Mammalian genome encodes seven sirtuin isoforms (SIRT1-SIRT7). Among them SIRT3 is the only isoform whose increased expression was linked to increased lifespan of humans. SIRT3 is primarily localized in mitochondria, where it regulates activity of many metabolic enzymes involved in ROS production and ATP biosynthesis. Because bioenergetic capacity of mitochondria is also dependent upon the fusion-fission dynamics of the organelle, this study was undertaken to study the effect of SIRT3 in regulating mitochondrial dynamics. We found that OPA1, an inner mitochondrial fusion protein was hyper-acetylated in hearts under pathological stress, including hearts with pressure overload hypertrophy, doxorubicin-induced cardiac toxicity and diabetic cardiomyopathy. OPA1 was also acetylated in SIRT3KO hearts, and this modification led to reduced GTPase activity of OPA1. In cardiomyocytes, SIRT3 was capable of deacetylating and elevating GTPase activity of OPA1. Moreover, SIRT3 overexpression prevented doxorubicin-mediated mitochondrial fragmentation and myocyte cell death by activating OPA1. In vivo studies conducted with SIRT3 overexpressing transgenic mice showed that SIRT3 protects the heart from developing cardiac hypertrophy and heart failure by preserving health of mitochondrial population. In summary, our data showed that SIRT3 promotes mitochondrial function not only by regulating activity of metabolic enzymes, but also by regulating mitochondrial dynamics by targeting OPA1. Based on this and other published data SIRT3 is considered a therapeutic target for the treatment of heart failure.

\section{3}

MODELING MECHANISMS OF DILATED CARDIOMYOPATHY BS Tuana

Department of Cellular and Molecular Medicine, University of Ottawa, Ottawa, Ontario

Dilated cardiomyopathy (DCM) is characterized by weak myocardium with inefficient pump action such that blood accumulates in the ventricles which dilate. DCM can arise due to exposure to toxic and infectious agents as well as deficits in metabolic performance of cardiac tissue. A significant portion $(\sim 30 \%)$ of DCM is associated with genetic mutations in genes encoding the cytoskeletal and contractile network as well as the nuclear lamina of cardiomyocytes. Much interest exists in modeling DCM in animals to potentially aid in clinical testing for human forms of the disease. In view of multifactorial nature of DCM and lack of knowledge for cause in a large $\%$ of cases, we have been interested in defining novel mechanisms that lead to DCM. In this regard we have generated two distinct mouse models of DCM. In the first model we sought to inhibit the E2F/Rb pathway by expressing the repressor E2F6 in postnatal myocardium. These mice presented with early onset DCM which was associated with major changes in gene expression and subcellular disorganization. In the second model we sought to alter the translation of the calcium signal by expressing a calcium/calmodulin dependent protein kinase II isoform (beta) in the postnatal heart. These mice also presented with DCM but with dysfunctional calcium handling proteins of the sarcoplasmic reticulum. The targeting of 
DCM via these two unique pathways will be addressed with the potential translation to human condition.

Supported by HSFC and CIHR.

\section{4}

\section{PDE5 INHIBITION IN PROTECTION OF DOXORUBICIN- INDUCED CARDIOMYOPATHY \\ RC Kukreja \\ Pauley Heart Center, Division of Cardiology, Virginia \\ Commonwealth University, Richmond, Virginia, USA}

Doxorubicin (DOX) is one of the most effective and commonly used chemotherapeutic agents for treating cancer in both children and adults. However, its clinical utility is limited due to cumulative cardiotoxicity that can lead to heart failure. Most of the cellular events triggered by DOX contribute to cell death which is the primary mechanism by which DOX induces cardiomyopathy. Several studies have shown that phosphoidesterase-5 (PDE5) inhibitors including sildenafil, vardenafil and tadalafil induce protective effect against ischemia/reperfusion injury in the heart. We also demonstrated that treatment with sildenafil prior to DOX treatment inhibited cardiomyocyte apoptosis, preserved mitochondrial membrane potential $(\Delta \psi \mathrm{m})$, myofibrillar integrity and prevented left ventricular (LV) dysfunction as well as ST segment prolongation. These effects were duplicated by the use of long acting PDE-5 inhibitor, Tadalafil which also prevented cardiomyocyte apoptosis in DOX-induced cardiomyopathy through mechanisms involving up-regulation of cGMP, increase in protein kinase $G$ activity and MnSOD level. Moreover, we observed that sildenafil is a powerful sensitizer of DOX-induced killing of prostate tumors in nude mice while providing concurrent cardioprotective benefit. These studies suggest that prophylactic treatment with PDE-5 inhibitors might become a promising therapeutic intervention for managing the clinical concern of DOX-induced cardiotoxicity in patients.

Supported in part by grants HL51045, HL59469, and HL79424 from the National Institutes of Health.

\section{5}

\section{NOVEL CARDIOPROTECTIVE ROLE OF MIR-133A}

\section{PK Mishra ${ }^{1,2}$}

${ }^{1}$ Department of Cellular and Integrative Physiology; ${ }^{2}$ Department of Anesthesiology, University of Nebraska Medical Center, Omaha, Nebraska, USA

MicroRNA-133a (miR-133a) is the most abundant miRNA in the heart. It is a cardioprotective miRNA that plays a crucial role in heart development and prevents cardiac hypertrophy and fibrosis in the adult heart. Downregulation of miR-133a is reported in failing human hearts and rodent diabetic hearts. Recently, we demonstrated that a reduced level of miR-133a is positively correlated with induction of cardiac autophagy (a process of sequestering damaged and aged cytoplasmic components into a double membrane vesicle called autophagosome and their subsequent degradation by lysosome) in diabetic heart failure patients undergoing mechanical unloading through left ventricle assist device. However, the miR-133a -autophagy axis is unclear in the heart. Important questions that need to be investigated to understand the specific role of miR-133a in cardiac autophagy include: whether the downregulation of miR-133a is a trigger for induced autophagy in diabetic hearts? If overexpression of miR-133a is able to restore cardiac autophagy? Which specific cargo is selected for miR-133a-mediated autophagy? To address these questions, I will elucidate the underlying molecular mechanism by which miR-133a regulates autophagy in diabetic hearts. I will also provide insight on a specific cargo selected for cardiac autophagy in diabetics, which is regulated by miR-133a. Further, I will discuss that restoration of basal level of cardiac autophagy by overexpressing miR-133a has potential to become a novel therapeutic target for diabetic heart failure. Acknowledgements: NIH grants: HL113281 and HL116205.
Session 2.12: Highlighting Sex Differences in Cardiovascular Diseases

\section{8}

\section{SEX DIFFERENCES IN CARDIAC TOLERANCE TO OXYGEN DEPRIVATION: DEVELOPMENTAL ASPECTS \\ B Ostadal \\ Institute of Physiology, Czech Academy of Sciences, Prague, \\ Czech Republic}

Human epidemiological studies have shown a clear association between the adverse intrauterine environments and an increased risk of ischemic heart disease in later adult life. Experimental studies have repeatedly suggested a possible link between early hypoxia and increased risk for cardiovascular disease in offspring. The question arises whether the effects of perinatal hypoxia on cardiac tolerance to ischemia differ in adult males and females. In the rat model, we have observed that the late myocardial effects of hypoxemia may be sex-dependent. Perinatal exposure to chronic hypoxia significantly increased cardiac tolerance to acute ischemia/reperfusion (I/R) injury - expressed as the lower incidence of ischemic arrhythmias - in adult female rats, while the opposite effect was observed in males. The mechanism of these late effects of perinatal hypoxia is currently unknown. The number of hypotheses includes prenatal sensitization of the apoptotic pathway, the lower expression of heat shock protein 70 in prenatal hypoxic hearts and decreased prenatal eNOS levels. Prenatal hypoxia induced sex-dependent changes in cardiac tolerance to ischemia in adults may be due to differences in fetal programming of the expression of the gene for $\mathrm{PKC} \varepsilon$, which plays a pivotal role in cardioprotection against $\mathrm{I} / \mathrm{R}$ injury: down-regulation of PKCe function was observed in the hearts of adult male offspring only. These experimental observations would have important clinical implications because cardiac sensitivity to oxygen deprivation in adult patients may be significantly influenced by perinatal disturbances in a sex-dependent manner.

\section{9}

\section{SEX-SPECIFIC IMMUNE MODULATION OF BLOOD PRESSURE} X Liu, H Ji, X Wu, K Sandberg

Department of Medicine and Center for the Study of Sex

Differences in Health, Aging and Disease, Georgetown University, Washington, DC, USA

Male wild type mice (WT-M) exhibit higher mean arterial pressure (MAP) than female wild type mice (WT-F) after angiotensin II (Ang II) infusion; however, in recombinant activation gene-1 null mice (Rag-1-/-), which lack the ability to produce mature $T$ cells, this sex difference in Ang II-induced hypertension was lost. After adoptive transfer of male CD3+, CD4+ or CD8+ $\mathrm{T}$ cells into male Rag-1//-mice (CD3M $\rightarrow$ Rag1-/--M; $\mathrm{CD} 4 \mathrm{M} \rightarrow \mathrm{Rag} 1 / /-\mathrm{M} ; \mathrm{CD} 8 \mathrm{M} \rightarrow \operatorname{Rag} 1 / /-\mathrm{M})$, the MAP was similar to WT-M levels. In contrast, adoptive transfer of female CD3+ and CD4+ T cells into the male Rag-1 / - host $(\mathrm{CD} 3 \mathrm{~F} \rightarrow \mathrm{Rag} 1-/-\mathrm{M}$; CD4F $\rightarrow$ Rag1-/ - M $)$ had no effect and adoptive transfer of female CD8 $+\mathrm{T}$ cells $(\mathrm{CD} 8 \mathrm{~F} \rightarrow \mathrm{Rag} 1-$ (--M) attenuated the magnitude of Ang II-induced hypertension. Taken together, these studies indicate that $\mathrm{T}$ cells modulate the development of hypertension in a sex-specific manner and that male $\mathrm{T}$ cells augment hypertension while female $T$ cells do not. To determine if this sex-specific effect of $T$ cells is due to intrinsic differences between male and female $T$ cells or to sex differences in exposure to gonadal hormones, Rag-1//--M mice were exposed to $800 \mathrm{rads}$ ( 8 Gy) using a 137 Cs irradiator (Georgetown University). Seven days prior to irradiation, Baytril $(0.17$ $\mathrm{mg} / \mathrm{mL}$ ) was added to the sterile drinking water of the recipient Rag-1//--M mice and the mice were maintained on this antibiotic supplemented water for an additional two weeks after irradiation to prevent infections. Four to six hours after irradiation, the mice received via retro-orbital injection $5 \mathrm{x}$ 107 (in $0.15 \mathrm{~mL}$ phosphate buffered saline) unfractionated bone marrow (BM) cells isolated from the femur and tibia from either WT-M or WT-F mice. We found a striking difference in survival after the bone marrow transplant. All of the mice receiving male BM cells died within 7 weeks of 
the transplant while all of the mice receiving female bone BM cells survived. Future BM transplantation studies are planned at lower irradiation doses to determine if sex-specific T cell effects on Ang II-induced hypertension is retained after BMT. Conclusion: Survival after bone marrow transplant in lethally irradiated recipient male mice is dependent upon the sex of the bone marrow donor, suggesting sex differences exist in BM cells that is independent of the male hormonal milieu in which they mature.

\section{0}

\section{ARGININE VASOPRESSIN IN PREECLAMPSIA}

JL Grobe

Department of Pharmacology \& Center for Hypertension Research, University of lowa, lowa City, lowa, USA

Preeclampsia is a hypertensive disorder of late pregnancy for which (despite having been recognized clinically for centuries) there remains no simple early-pregnancy diagnostic biomarker tests, no simple and effective animal models of the early pathogenesis, and the only true 'cure' remains the (often premature) delivery of the fetus and placenta. Compared to a normal pregnancy, preeclampsia represents a low-renin subtype of hypertension, which led us to hypothesize a role for arginine vasopressin (AVP) in its pathogenesis. Copeptin, the stable / slow-clearance C-terminal portion of the AVP gene product that is released in a 1:1 molar ratio to AVP, was found to be grossly elevated in first-trimester plasma samples from women that eventually developed preeclampsia, and its levels were found to be very strongly predictive of the disorder as early as the 6th week of gestation (ROC AUC $=0.90$, or 0.96 for women with no history of preeclampsia). Chronic infusion of AVP (24 ng/hr, sc) during gestation in wildtype $\mathrm{C} 57 \mathrm{BL} / 6 \mathrm{~J}$ mice resulted in pregnancy-specific hypertension, renal glomerular endotheliosis, proteinuria, intrauterine growth restriction. We conclude that elevated AVP/copeptin precedes the development of preeclampsia, is strongly predictive of the disorder in very-early gestation, and is sufficient to induce symptoms of preeclampsia. Ongoing work is focused on identifying the receptor subtypes and timing of exposure which mediate AVP-induced maternal preeclampsia symptoms, the role of elevated AVP in programming of adult cardiometabolic dysfunction in offspring from preeclamptic pregnancies, and interactions between AVP and genetic risk factors that may synergistically precipitate the development of preeclampsia.

\section{1}

\section{MYOCARDIAL PROTECTION ISSUES IN THE NEWBORN:} SEX, STRESS AND OXYGEN

\section{Wittnich}

Departments of Surgery \& Physiology, University of Toronto,

Toronto, Ontario

Newborn children undergoing congenital cardiac surgery are known to have worse outcomes than older children. This presentation will discuss our research program exploring reasons for this including that newborn hearts tolerate ischemia less well than adults, develop irreversible injury sooner and exhibit at risk metabolic profiles. For example, neonatal hearts have a greater vulnerability to acid-base disturbances during ischemia due to less buffering capacity rendering them more susceptible to injury during ischemia. Additionally, ventricular dysfunction is reported greater in the left (LV) versus right ventricle (RV) in infants following surgically induced ischemia. Our research documents significant differences in LV enzyme activities and metabolic profiles that lead to worse acidosis and lower energy levels during ischemia; offering one reason for the greater LV-dysfunction relative to the RV. Children can also be exposed to high oxygen levels (hyperoxia) for hours to days during their medical and/or surgical management. Our research shows that hyperoxia in the newborn triggers oxygen free radical-mediated membrane injury because of an inability of the newborn heart to upregulate its' antioxidant enzyme defences while impairing myocardial function and hemodynamics. Finally, recent reports in children with congenital heart disease, female sex has been linked to greater in-hospital mortality associated with low cardiac output, yet the reasons for this are unclear. We have identified, compared to males, the hearts of newborn females are at a metabolic disadvantage - having even lower energy levels and greater tissue lactic acidosis, both linked to an increase susceptibility to ischemic injury and impair myocardial function upon reperfusion.
Session 3.1: Dennis B McNamara

Symposium: Pathophysiology and Treatment of Peripheral Vascular Disease

\section{6}

\section{EFFECTIVENESS OF ARTIFICIAL CARBON DIOXIDE FOOT BATH FOR MEDICAL THERAPY; SUMMARY AND META- ANALYSIS OF JAPANESE PUBLISHED STUDIES AND CASE REPORTS FOR PAST 15 YEARS}

H Hasebe $^{1}$, H Kumamoto ${ }^{2}$, D Hawkins ${ }^{3}$

${ }^{1}$ Mitsubishi Rayon America Inc., New York, New York, USA; ${ }^{2}$ Mitsubishi Chemical Holdings, Tokyo, Japan; ${ }^{3}$ School of Statistics, University of Minnesota, Minneapolis, Minnesota, USA

Carbon dioxide foot bath has been on market for last 20 years in Japan and many studies and case reports had been published and knowledge was shared in Japanese Medical Communities. However these studies and case reports were published only in Japanese, so they are not shared or appreciated in outside of Japan. Total 307 academic publications related to Carbon Dioxide Foot Bath Treatment had been published from 1999 to 2014 with total subjects number of 2,708. Especially Publications related to "Carbothera" Hallow membrane Carbon dioxide generator with Foot bath were total 48 publications total subjects/patients number was 825 . We summarized these publications and performed meta-analysis results to evaluate effectiveness of artificial CO2 spring water foot bath treatment.

\section{7}

SKIN PERFUSION PRESSURE AND WOUND CLOSURE TIME IN LOWER EXTREMITY WOUNDS IN DIABETIC AND NON-DIABETIC PATIENTS

\section{K Suzuki}

Cedars-Sinai Medical Center, Los Angeles, California, USA

Peripheral Arterial Disease (PAD) affects approximately 8 million patients in the United States. We investigated the relationship of Skin Perfusion Pressure (SPP) and wound closure time in lower extremity wounds due to limb ischemia. We conducted a retrospective study of 1125 lower extremity wounds in 998 patients between June 2006 and October 2014. We analyzed the relationship between SPP and wound closure time. SPP was measured using a Laser Doppler instrument. Patients with SPP values over $30 \mathrm{mmHg}$ had shorter wound closure times, while patients with SPP values below $30 \mathrm{mmHg}$ had a significantly longer wound closure time. Diabetic patients took longer to achieve wound closure compared to non-diabetics. No significant relationship was observed between SPP and wound closure time in relation to age or gender. SPP is a useful tool in estimating time to wound closure and assessing the necessity of vascular interventions in lower extremity wounds due to PAD.

\section{Session 3.2: Diastolic Heart Failure with Preserved Ejection Fraction: Mechanism and Therapy}

\section{8}

\section{DEPRESSED SARCOPLASMIC RETICULUM ACTIVITY} UNDERLIES $\mathrm{CA}^{2+}$ DYSHOMEOSTASIS IN A RAT MODEL OF METABOLIC SYNDROME: ROLE OF PHOSPHODIESTERASES B Turan

Department of Biophysics, Ankara University Faculty of Medicine, Ankara, Turkey

High-carbohydrate diet-induced obesity and/or metabolic syndrom (MetS) is recognized as an independent risk factor for dysfunctioning of the heart. Although several mechanisms have been postulated for its identification, no precise mechanism is available yet for the cardiac remodeling and contractile dysfunction under MetS. Therefore, we aimed to investigate the 
role of sarcoplasmic reticulum (SR) on altered intracellular $\mathrm{Ca}^{2+}$ regulation in cardiomyocytes from MetS male rats, induced with high-sucrose drinking water during 16-week period. Basically, electrically-stimulated $\mathrm{Ca}^{2+}$ transients and L-type $\mathrm{Ca}^{2+}$-currents (LTCC) were recorded in ventricular myocytes from MetS rats relative to myocytes from control rats. We also investigated SR-Ca ${ }^{2+}$-ATPase (SERCA) activity as well as cardiac ryanodine receptor (RyR2) family proteins' phosphorylation levels by Western blot analysis. The $\mathrm{Ca}^{2+}$ transients exhibited significantly reduced amplitude $(\sim 30 \%)$, and prolonged time courses ( 2 -fold) with no change in LTCC as well as depressed $\mathrm{Ca}^{2+}$ loading of SR ( $\left.55 \%\right)$ due to caffeine responses in cardiomyoctes from MetS rats with increased basal $\mathrm{Ca} 2+$ compared to aged-matched control rats. The data with caffeine responses under $\mathrm{ONaOCa}$ also demonstrated a depressed SERCA activity in MetS cardiomyocytes which was further supported with the data on a slowed cytosolic $\mathrm{Ca}^{2+}$ removal associated with a significant decrease in SERCA2. mediated $\mathrm{Ca}^{2+}$ reuptake. Additionally, the data with tetracaine application assay was supporting a leaky-RyR2 in cardiomyocytes from MetS group. Moreover, high phosphorylation levels of both RyR2 and phospholamban are supporting a depressed SR activity which underlies $\mathrm{Ca}^{2+}$ dyshomeostasis in MetS rat model. Furthermore, we found that MetS induced cardiac dysfunction through myofibril loss, connective tissue and lipid accumulation in the myocardium. Moreover, our study showed that cardiac expressions and activities of some phosphodiesterases (PDEs) increased in MetS and, consistent with these results, the effects of PDE inhibitors on the pathways that control cardiac contraction were higher than those in the control group. In conclusion, we established that a 16-wk high sucrose feeding protocol, which induced cardiac dysfunction, leads to Met $S$ disease model in rats. We define the components of MetS-induced cardiac dysfunction as follows: structural changes in myocardium, $\mathrm{Ca} 2+$ dyshomeostasis and increased PDE activity. The most striking result of this study is, in the absence of type- 2 diabetes or obesity, MetS induced by a high-sucrose diet was enough to alter cardiac performance in the rats. The presence of insulin resistance or MetS should be taken into account in connection with the clinical use of some PDE inhibitors to avoid their potential side effects. Acknowledgements: TUBITAK SBAG-113S466.

\section{9}

\section{EVALUATION OF QT DISPERSION IN ASYMPTOMATIC PATIENTS WITH GRADE I LEFT VENTRICULAR DIASTOLIC DYSFUNCTION}

$\underline{\text { AO Blackman }}{ }^{1,2}$, J Sobral Neto ${ }^{1}$, OM Gomes ${ }^{1}$

${ }^{1}$ Centrocard - Cardiologic Evaluation Center, Brasilia;

${ }^{2}$ Cardiovascular Foundation S Francis of Assisi - ServCor, Belo Horizonte, Brazil

INTRODUCTION: Grade I left ventricular diastolic dysfunction, a mechanical phenomenon related to abnormalities of ventricular relaxation, is highly prevalent in the population and leads to over heart failure. Furthermore, QT dispersion (QTD $\geq 80 \mathrm{~ms}$ ), an electrical phenomenon, is related to significantly increased risk of severe arrhythmias and sudden cardiac death.

OBJECTIVE: To analyze the influence of grade I left ventricular diastolic dysfunction on QT dispersion in asymptomatic patients estimating the circadian variation of QT dispersion and the incidence of arrhythmia in the population. Method: Consisted of assessing 26 patients with grade I left ventricular diastolic dysfunction and average ages between $55.7 \pm 5.8$, and presenting arterial hypertension $34 \%$, type II diabetes $7 \%$, sedentary $27 \%$, smokers $12 \%$, and did not show any prior knowledge of cardiac disease $20 \%$. Patients underwent a 24 hour ambulatory recording, using a 12 leadHolter device to measure QT dispersion during four periods: morning, afternoon, evening and night during sleep. It was also evaluated the prevalence of arrhythmias.

RESULTS: The average heart-rate-corrected QT was $71.7 \pm 13.3,69 \pm 9.8$, $68,2 \pm 9.6$, and $65.1 \pm 9.6 \mathrm{~ms}$, morning, afternoon, evening and night during sleep respectively. Nevertheless, in $30.8 \%$ of the patients it was found average heart-rate-corrected $\geq 80 \mathrm{~ms}$. One patient $(3.8 \%)$ presented premature ventricular complex higher than $10 / \mathrm{h}$.

CONCLUSION: This research demonstrated the occurrence of QT interval dispersion in $30.8 \%$ of patients without cardiovascular symptoms, with grade I left ventricular diastolic dysfunction.
050

\section{UTILIZING MATHEMATICAL MODELING AND ECHOCARDIOGRAPHY SPECKLE TRACKING TO UNDERSTAND FUNCTION OF THE LEFT VENTRICLE IN HFPEF}

MJ Moulton ${ }^{1}$, B Hong ${ }^{2}$, TW Secomb ${ }^{2}$

${ }^{1}$ Department of Surgery, University of Nebraska Medical Center, Omaha, Nebraska; ${ }^{2}$ Department of Physiology, University of Arizona, Tucson, Arizona, USA

Heart failure with a preserved ejection fraction (HFpEF) is still underdiagnosed and the pathophysiology is debated in patients. Part of the problem with better characterizing the pathophysiology is difficulty assessing details of heart function in patients. Tissue samples and invasive testing are not readily available in patients. Accordingly, we have developed a non-invasive modeling methodology that couples a low order model of the LV with echocardiography speckle tracking. Myocardial strains, stresses and systolic and diastolic properties are estimated and compared with normal volunteers. Eleven patients with $\mathrm{HFpEF}$ and eleven volunteers were studied with echocardiography, models were constructed and LV properties determined. Continued improvement in echo techniques coupled with advanced modeling methods may offer insight into a very heterogeneous disease and may allow risk stratification for patients with HFpEF.

\section{1}

\section{MMP-9-MEDIATED MECHANISMS OF DIASTOLIC DYSFUNCTION}

\section{Lindsey}

Mississippi Center for Heart Research, Department of Physiology and Biophysics, University of Mississippi Medical Center, and Research Service, GV (Sonny) Montgomery Veterans Affairs Medical Center, Jackson, Mississippi, USA

Following myocardial infarction (MI), the left ventricle (LV) undergoes a series of cardiac wound healing responses that involve both the stimulation of robust inflammation to clear necrotic myocytes and tissue debris and the induction of extracellular matrix (ECM) protein synthesis to generate an infarct scar. Collectively, this process in known as LV remodeling. Matrix metalloproteinase-9 (MMP-9) is a key regulator of LV remodeling post-MI, through direct effects on ECM turnover as well as indirect effects on the regulation of the major cell types that coordinate cardiac wound healingnamely the infiltrating leukocytes and the cardiac fibroblasts. We will discuss recent research that has expanded our understanding of post-MI LV remodeling, including recent proteomic advances focused on the ECM compartment to provide novel functional and translational insights. In summary, this talk will provide an overview of how cardiac ECM research has evolved over the last decade and will provide insight into future directions that will drive our understanding of MMP-9 directed cardiac ECM turnover in the post-MI LV.

Funding Sources: NIH HHSN 268201000036C (N01-HV-00244) for the San Antonio Cardiovascular Proteomics Center, HL075360, HL051971, GM114833, and GM104357; and the Biomedical Laboratory Research and Development Service of the Veterans Affairs Office of Research and Development Award 5101BX000505

\section{Session 3.3: Kern Wildenthal Young Faculty} Competition (Translational Medicine)

\section{2}

SECRETORY CYCLOPHILIN A AS A POTENTIAL SEROLOGICAL MARKER OF MACROVASCULAR DISEASE IN TYPE 2 DIABETES MELLITUS

\section{S Ramachandran, A Vinitha, CC Kartha}

Rajiv Ghandi Centre for Biotechnology, Trivandrum, India

Diabetes is a proinflammatory state and is characterized by elevated plasma cytokines, chemokines, adhesion molecules and monocytic activity. Cellular events such as monocyte activation are intensified in type 2 
diabetes mellitus (T2DM) leading to accelerated development of atherosclerotic vascular lesions.

We analyzed the monocyte proteome for potential markers of early atherogenesis in T2DM using 2DE/MS/MS. We identified Cyclophilin A, an immunophilin secreted from monocytes activated by hyperglycaemia. Plasma levels of cyclophilin A were assayed in 556 subjects including normal healthy volunteers and patients with T2DM, with or without coronary artery disease (CAD). Plasma Cyclophilin levels were higher in diabetes patients with or without CAD compared to normal subjects $(\mathrm{P}<0.001)$. Age, fasting blood sugar levels and $\mathrm{HbA1C}$ levels were positively associated with increased plasma cyclophilin. Cyclophilin levels were elevated in patients with increased serum CRP levels $(\mathrm{p}=0.016)$ and reduced in patients using metformin $(\mathrm{p}<0.001)$. There was a strong association of high cyclophilin values with diabetes and vascular disease $(p<0.001)$. Gene variants of cyclophilin did not affect the plasma protein levels.

We further studied the mechanistic role of cyclophilin in initiation of atherogenesis in diabetes. Extracellular cyclophilin acts as a chemoattractant for monocytes and promotes monocyte endothelial adhesion. Foam cell formation, the hallmark of early atherogenesis also increases in macrophages cultured with Cyclophilin A. Cyclophilin A is currently being evaluated as a marker of proinflammatory status in type 2 diabetes and possibly a predictor of early vascular disease through a prospective study. Acknowledgements: Indian Council for Medical Research, Government of India

\section{3}

\section{TARGETING HOST IMMUNE RESPONSE TO ENHANCE EFFICACY OF ALLOGENEIC MESENCHYMAL STEM CELLS FOR CARDIAC REGENERATION}

\section{S Dhingra}

Regenerative Medicine Program, Institute of Cardiovascular Sciences, St Boniface Research Centre, University of Manitoba, Winnipeg, Manitoba

Allogeneic mesenchymal stem cells (MSCs) are immunoprivileged, therefore these cells can suppress host immune system and survive after transplantation. However recent data from preclinical and clinical studies indicate that allogeneic MSCs provoke immune response in the recipient and are rejected after transplantation. We confirmed that allogeneic MSCs improved heart function after implantation in the infarcted heart. However, late after implantation, the cells lost their immunoprivilege, and were rejected. Therefore we started extensive investigations to study mechanisms of rejection, our data demonstrate that immunoprivilege of MSCs was maintained by prostaglandin E2 (PGE2) produced by these cells. We further demonstrated that myogenic differentiation of MSCs led to PGE2 decrease and loss of immunoprivilege. PGE2 treatment of differentiated MSCs preserved immunoprivilege. Using an in vivo rat model, MSCs $(3 \times 106$ cells/rat $)$ with or without a biodegradable hydrogel that slowly released PGE2 were injected into infarct region. After 5 weeks significant number of transplanted cells survived in hydrogel+PGE2 group compared to control group. In conclusion, maintaining PGE2 levels in differentiated MSCs preserved immunoprivilege and restored cardiac function after MI.

\section{4}

\section{LOX-1 DELETION ATTENUATES BRAIN INJURY FOLLOWING CHRONIC MYOCARDIAL ISCHEMIA}

\section{Wang, V Manavalan, Z Ding, Y Dai, JL Mehta}

Central Arkansas Veterans Healthcare System and the University of Arkansas for Medical Sciences, Little Rock, Arkansas, USA

Ischemic heart disease is the most cause of morbidity and mortality. It is generally thought, but not proven that myocardial ischemia affects brain function. LOX-1, a lectin-like receptor for oxidized low density lipoprotein (ox-LDL), is responsible for the binding and uptake of ox-LDL as well as other ligands exhibiting oxidized phospholipids. Previous studies have demonstrated that blockade or deletion of LOX-1 reduces ischemic myocardial injury in mice. Whether myocardial ischemia per se affects brain function and whether LOX-1 expression is involved in this process are not known. In this study, WT C57BL/6J mice and LOX-1 KO mice were exposed to chronic myocardial ischemia by total ligation of left coronary artery (LCA) for 4 weeks. The sham operated WT mice and LOX-1 KO mice served as controls. Cardiac remodeling and function were analyzed by hematoxylin and eosin (HE) staining, Masson Trichrome (MT) staining and echocardiographic analysis, and the brain injury was analyzed by HE staining, cresyl violet (CV) staining and TUNEL assay. In accordance with previous studies, we observed in this study that LOX-1 deletion reduced LCA occlusion-induced myocardial damage and fibrosis, and limited deterioration of cardiac function. More interestingly, there was a significant increase in damaged and apoptotic neurons in the cerebral cortex of WT mice starting at 1 week after LCA occlusion and persisting for 4 weeks. Hypoxic and apoptotic neurons were much less in the brains of LOX-1 KO mice than in WT mice. However, the mechanism by which myocardial ischemia induces brain injury is not known, but it is clear that LOX-1 deletion reduces brain injury.

\section{5}

EXERCISE TRAINING ATTENUATES CHEMOREFLEX-MEDIATED REDUCTIONS OF RENAL BLOOD FLOW IN HEART FAILURE NJ Marcus ${ }^{1,2}$, C Pügge ${ }^{2}$, J Mediratta ${ }^{2}$, AM Schiller ${ }^{2}$, R Del Rio ${ }^{3}$, IH Zucker ${ }^{2}$, HD Schultz ${ }^{2}$

${ }^{1}$ Department of Physiology and Pharmacology, Des Moines University, Des Moines, lowa; ${ }^{2}$ Department of Cellular and Integrative Physiology, University of Nebraska Medical Center, Omaha, Nebraska; ${ }^{3}$ Laboratory of Cardiorespiratory Control, Universidad Autónoma de Chile, Sede Temuco, Chile In chronic heart failure (CHF), carotid body chemoreceptor (CBC) activity is increased and contributes to increased tonic and hypoxia-evoked elevation in renal sympathetic nerve activity (RSNA). Elevated RSNA and reduced renal perfusion may contribute to development of the 'cardiorenal' syndrome in CHF. Exercise training (EXT) has been shown to abrogate $\mathrm{CBC}$-mediated increases in RSNA in experimental heart failure; however the effect of EXT on CBC control of renal blood flow (RBF) is undetermined. We hypothesized that $\mathrm{CBCs}$ contribute to tonic reductions in $\mathrm{RBF}$ in $\mathrm{CHF}$, that stimulation of the $\mathrm{CBC}$ with hypoxia would result in exaggerated reductions in RBF, and that these responses would be attenuated with EXT. RBF was measured in 1) CHF-sedentary (SED), 2) CHFEXT, 3) CHF-carotid body denervation (CBD), and CHF-renal denervation (RDNX) groups. We measured RBF at rest and in response to hypoxia ( $\mathrm{FiO} 210 \%)$. All animals exhibited similar reductions in ejection fraction and fractional shortening as well as increases in ventricular systolic and diastolic volumes. Resting RBF was lower in CHF-SED $(29 \pm 2 \mathrm{ml} /$ $\mathrm{min}$ ) compared to CHF-EXT animals $(46 \pm 2 \mathrm{ml} / \mathrm{min}, \mathrm{p}<0.05)$, and compared to CHF-CBD animals $(42 \pm 6 \mathrm{ml} / \mathrm{min}, \mathrm{p}<0.05)$. In CHF-SED, RBF decreased during hypoxia, and this was prevented in CHF-EXT animals. Both CBD and RDNX abolished the RBF response to hypoxia in CHF. MAP increased in response to hypoxia in CHF-SED, but was prevented by EXT, CBD, and RDNX. EXT is effective in attenuating chemoreflexmediated tonic and hypoxia-evoked reductions in RBF in CHF.

\section{Session 3.4: New Concepts in Arrhythmogenesis}

\section{6 \\ NEW ASPECTS OF REGULATION OF CARDIAC ACTION POTENTIAL DURATION}

PP Nánási

Department of Physiology, University of Debrecen, Hungary

In this talk three recently described features of regulation of action potential duration (APD) are discussed, each based on changes in the net membrane current (Inet) during the plateau of the AP.

1. The reverse rate-dependent nature of drug effects on APD means that any drug-induced change in APD is more pronounced at longer than at shorter cycle lengths. Similar results are obtained when repolarization is modified by injection of inward or outward current pulses. On the other hand, all drug-induced or current-induced changes in APD well correlate with the baseline value of APD in all mammalian preparations studied, 
including the human heart. Since Inet is inversely proportional to APD, and consequently to cycle length, it is concluded that that reverse ratedependency may simply reflect the inverse relationship linking Inet to APD. In summary, reverse rate-dependency is an intrinsic property of cardioactive drug actions.

2. Beat-to-beat variability (short-term variability, SV) of APD is a good predictor of cardiac arrhythmias, however, the factors influencing its magnitude are not fully clarified.

In order to eliminate the direct effects of APD changes on SV, relative SV (RSV) was introduced by normalizing the observed SV-changes to concomitant APD-changes and comparing this ratio to the exponential SV-APD relationship, obtained using inward and outward current injections. RSV is decreased by ion currents playing critical role in the negative feedback regulation of APD, such as $\mathrm{ICa}$, IKs and $\mathrm{IKr}$, therefore blocking of these currents may carry some proarrhythmic risk. Conversely, RSV is increased by INa, in line with the known antiarrhythmic effect of late INa blockade. RSV is modulated by several further parameters, like intracellular $\mathrm{Ca} 2+$ concentration, tissue redox potential, stimulation rate or temperature.

3. Adrenergic activation of L-type $\mathrm{Ca}^{2+}$ and various $\mathrm{K}^{+}$currents is a crucial mechanism of cardiac adaptation, however, it carries substantial proarrhythmic risk. It was found that the isoproterenol (ISO)-induced activation of ICa precedes the enhancement of IKs and IKr. Since this temporal shift is differently affected by selective blockade of $\beta 1$ and $\beta 2$ adrenoceptors, and is reduced after inhibition of phosphodiesterases, different adrenergic signal transduction pathways and compartmentalization is likely involved.

\section{7}

\section{LQT5 TRANSGENIC RABBITS: A NEW MODEL EXHIBITING INCREASED CARDIAC REPOLARIZATION INSTABILITY AND ARRHYTHMIA SUSCEPTIBILITY}

\author{
I Baczkó ${ }^{1}$, P Major ${ }^{2}$, V Juhász ${ }^{1}$, R Varga ${ }^{1}$, T Hornyik ${ }^{1}$, L Hiripi ${ }^{2}$,
} Z Bösze $^{2}$, A Varró ${ }^{1,3}$

${ }^{1}$ Department of Pharmacology and Pharmacotherapy, University of Szeged, Szeged; ${ }^{2}$ Agricultural Biotechnology Center, Gödöllö;

${ }^{3}$ MTA-SZTE Research Group of Cardiovascular Pharmacology, Hungarian Academy of Sciences, Szeged, Hungary

The reliable prediction of proarrhythmic risk associated with novel compounds under development remains elusive and new models with better predictive value are needed. We performed the primary characterization of a new transgenic LQT5 rabbit model, recently generated by our group, based on selective cardiac overexpression of a mutant human KCNE1 gene. This missense mutation was first described in a Chinese LQT syndrome family. Arrhythmias were provoked by i.v. administration of the IKr blocker dofetilide in LQT5 transgenic (TG) rabbits and wild type(WT) littermates. The ECG was continuously registered, and arrhythmia development was monitored. Conventional ECG parameters characterizing repolarization duration, the QT and frequency corrected QT intervals (QTc), were not different in the two groups at baseline (QT: $146.9 \pm 3.18$ in WT vs. $145.7 \pm 2.87 \mathrm{~ms}$ in TG; QTc: $156.8 \pm 2.63$ in WT vs. $155.6 \pm 2.12 \mathrm{~ms}$ in $\mathrm{TG}, \mathrm{n}=26$ and 27 , all $\mathrm{p}>0.05)$. Following dofetilide, QT and QTc intervals were significantly prolonged in both groups (QTc: 169.4 \pm 3.37 in WT and $167.8 \pm 3.17 \mathrm{~ms}$ in TG). However, the short-term variability of the QT interval, an ECG parameter suggested for better estimation of proarrhythmic risk, was higher in KCNE1 TG rabbits compared to WT at baseline $(4.8 \pm 0.26$ in TG vs. $2.8 \pm 0.15 \mathrm{~ms}$ in WT, $\mathrm{p}<0.05)$. Following dofetilide, the incidence of the typically drug-induced Torsades des Pointes arrhythmia was significantly higher in TG rabbits compared to WT ( $77 \%$ vs. $51.8 \%$, p $<0.05)$. In conclusion, KCNE1 transgenic LQT5 rabbits have increased susceptibility to drug-induced arrhythmias and may be suitable for more reliable testing of the proarrhythmic potential of novel compounds under development.

\section{8}

\section{ANTIARRHYTHMIC AND CARDIAC ELECTROPHYSIOLOGIC EFFECT OF DESETHYLAMIODARONE}

\section{A Varró}

University of Szeged, Department of Pharmacology and

Pharmacotherapy, Szeged, Hungary

Desethylamiodarone (DEA) is the major metabolite of amiodarone (AMIO), which is considered as one of the most effective drugs for the treatment of various types of cardiac arrhythmias including atrial fibrillation $(\mathrm{AF})$. The therapeutic value of AMIO, however, is greatly limited by its non-cardiac adverse effects. Previous reports indicated that DEA had more binding affinity for the cardiac thyroid receptor than its parent compound AMIO. Therefore, the aim of our study was to compare the possible antiarrhythmic effect of DEA in comparison with AMIO in different experimental arrhythmia models. In a conscious rat model of coronary artery ligation-induced ventricular arrhythmias, per os $50 \mathrm{mg} / \mathrm{kg} /$ day (3 weeks) DEA administration exerted similar antiarrhythmic effects to 100 $\mathrm{mg} / \mathrm{kg} /$ day (3 weeks) AMIO pre-treatment increasing survival from $23 \%$ $(n=39)$ to $80 \%$ and $75 \%$, respectively. In the atrial tachypaced dog atrial fibrillation model, per os $50 \mathrm{mg} / \mathrm{kg} /$ day (4 weeks) AMIO treatment decreased the duration of AF episodes to a similar extent to per os $25 \mathrm{mg} /$ $\mathrm{kg} /$ day (4 weeks) DEA treatment. In the cellular level chronic DEA treatment - similarly to that of AMIO - increased cardiac ventricular action potential duration and frequency dependently decreased the maximal rate of depolarization (Vmax). On the surface ECG significant QTc lengthening was observed also after both AMIO and DEA treatments. After chronic treatment with AMIO and DEA the corresponding tissue (lung, liver and brain) drug level measurements yielded smaller drug levels with DEA compared to that of AMIO. Based on these results it can be expected that using DEA instead of AMIO would result in similar therapeutic effects with possibly reduced drug toxicity.

\section{9}

FIBROSIS: A STRUCTURAL MODULATOR OF ATRIAL PHYSIOLOGY AND ARRHYTHMIAS IN THE HUMAN HEART VV Fedorov, BJ Hansen, TA Csepe

Department of Physiology \& Cell Biology and Davis Heart \& Lung Research Institute, The Ohio State University Wexner Medical Center, Columbus, Ohio, USA

Atrial fibrillation (AF) is the most common sustained arrhythmia and is associated with increased cardiovascular morbidity and mortality. Despite several decades of extensive research, gaps in understanding the mechanisms of AF still exist, making successful treatment particularly challenging. Electrophysiological and structural (fibrosis) abnormalities have been shown to underlie AF development. However, the contribution of the three dimensional (3D) atrial microstructure, including heterogeneous fibrosis distribution, to both the development and persistence of AF in the diseased human atria has not been clearly elucidated. Importantly, the 3D microstructural substrates of AF in diseased human hearts cannot be directly reproduced in any animal model and currently cannot be studied in-vivo. For this purpose, we developed a novel approach to simultaneously map sub-endocardial (sub-Endo) and sub-epicardial (sub-Epi) activation patterns and integrate these data with ex vivo 3D gadolinium-enhanced MRI images of the atrial microanatomic architecture, including fibrosis, in order to elucidate possible mechanisms of AF in diseased human hearts. Our pilot data show that the primary mechanism of AF maintenance in human hearts is a limited number of patient-specific AF drivers (intramural reentries) anchored to microanatomic substrates with predictable structural features, such as enhanced interstitial fibrosis, atrial bundle thickness, and high Sub-endo vs Sub-epicardial myofiber angle differences. We suggest that the use of explanted human hearts combined with our novel integrative 3D functional/structural approach will uncover the localized pathologic microanatomy of the human heart that is responsible for AF and other arrhythmias, ultimately leading to new treatment strategies of these heart rhythm disorders. 
Session 3.5: George Jackowski Symposium Prevention of Heart Disease

060

CANDIDATE GENE TARGETING REVEALS THE FUNCTIONAL ROLE OF MYOCARDIN IN DILATED CARDIOMYOPATHY

M Khullar, A Mittal, S Rana ${ }^{5}$, R Sharma ${ }^{1}$, A Kumar' ${ }^{2}$, SK Raut ${ }^{2}$, V Arige $^{6}$, S Sharma ${ }^{5}$, R Prasad ${ }^{2}$, UN Saikia ${ }^{3}$, S Sarkar ${ }^{5}$, NR Mahapatra ${ }^{6}$, A Bahl $^{1}$

${ }^{1}$ Department of Cardiology; ${ }^{2}$ Department of Experimental Medicine and Biotechnology; ${ }^{3}$ Department of Histopathology; ${ }^{4}$ Department of Internal Medicine, Post Graduate Institute of Medical Education and Research, Chandigarh, India; ${ }^{5}$ Department of Zoology,

University of Calcutta, India, ${ }^{6}$ Department of Biotechnology, Indian Institute of Technology, Madras, India

Dilated cardiomyopathy (DCM) is an important cause of heart failure (HF) and sudden cardiac death and a leading indication for cardiac transplantation. To combat the very poor outcome of HF in general and DCM in particular, it is critical to better understand the pathophysiology of DCM. Myocardin, a cardiac-specific transcription factor, has been shown to be increased in end stage HF, and in DCM patients. We examined role of Myocardin in DCM and in Renal artery ligation (RAL) model of cardiomyopathy. We found increased cardiac Myocardin expression in DCM patients and in RAL model of cardiomyopathy. In vitro silencing of Myocardin by siRNA resulted in decreased expression of sarcomeric genes (troponin I, troponin T and cardiac actin), hypertrophy marker, ANP and fibrotic genes (Col1a, Col3a, Col4a, CTGF, TGF- $\beta$ and FGF- $\beta$ ) in cardiomyocytes and cardiac fibroblasts respectively. Cardiac specific Inhibition of Myocardin using a homing peptide in RAL model of cardiomyopathy resulted in decreased cardiac expression of hypertrophy markers (ANP and $\beta$-MHC), fibrotic markers (Colla, Col3a, Col4a, CTGF, TGF- $\beta$ and FGF$\beta)$ and attenuated cardiac hypertrophy and fibrosis. We investigated the upstream regulatory mechanisms controlling the expression of Myocardin and identified miRNA-33a as a regulator of Myocardin expression. We propose that Myocardin is involved in DCM associated cardiac remodeling and Myocardin gene targeting offers a novel therapeutic target in DCM.

\section{1}

\section{UPDATE ON INFECTIVE ENDOCARDITIS}

\section{E Castañeda Saldaña}

Cayetano Heredia Peruvian University, Lima, Peru

Infective endocarditis (IE), a microbial infection of the endocardial surface of the heart, has been classified as acute or subacute-chronic. The epidemiologic features of infective endocarditis are changing as a result of increasing longevity, new predisposing factors, and an increase in nosocomial cases. Mitral-valve prolapse is now the most common cardiovascular diagnosis predisposing patients to infective endocarditis. Other conditions associated with an increased incidence of infective endocarditis include poor dental hygiene, long-term hemodialysis, diabetes mellitus and infection with the human immunodeficiency virus (HIV). The modified Duke criteria provide the basis for the diagnosis of infectious endocarditis; also include recommendations for choosing between transthoracic echocardiography (TTE) and transesophageal echocardiography (TEE). TEE is more sensitive than TTE for defining perivalvular extension of infective endocarditis and the presence of a myocardial abscess and valve perforations. Congestive heart failure and neurologic events have the greatest influence on the prognosis of infective endocarditis. It has not been demonstrated benefits of anticoagulant therapy. Combined medical and surgical therapy for infective endocarditis can decrease mortality among patients who have congestive heart failure, perivalvular invasive disease, or uncontrolled infection despite maximal antimicrobial therapy; congestive heart failure is the strongest indication for surgery in infective endocarditis. A recent neurologic complication of infective endocarditis has been considered a relative contraindication to valve-replacement surgery. Early surgery for native valve endocarditis is associated with an in-hospital mortality benefit compared with medical therapy alone.

\section{2}

\section{MOLECULAR SIGNATURES AS TOOLS FOR MANAGEMENT OF ORGAN FAILURE}

\section{B McManus}

PROOF Centre of Excellence, Vancouver, British Columbia

Heart, lung and kidney diseases together constitute a massive and growing burden that amounts to over $\$ 3$ trillion of health care spending worldwide. Through non-targeted identification of omics biomarker signatures (transcripts, proteins, metabolites) and development of relevant signatures into actionable blood tests, the Prevention of Organ Failure (PROOF) Centre of Excellence aims to produce a new generation of patient-specific laboratory tests to better guide patient care and to identify those at risk of organ failure. In our lead program, Biomarkers in Transplantation (BiT), we are now implementing a discriminative blood test that determines which heart transplant patients are not undergoing acute rejection and thus need not receive biopsies. The biopsy is the "gold standard" for detecting rejection, but is an expensive, highly invasive procedure that may produce inconclusive results. Our previous BiT1 and BiT2 studies used Affymetrix microarrays to measure whole blood mRNA levels in heart transplant patients, and identified a promising 40-mRNA signature for excluding acute rejection with high negative predictive value (NPV). In BiT3, these signatures were developed on two clinically amenable gene-expression platforms: HTG EDGE-Seq and NanoString nCounter. Using a discovery cohort of 38 patient samples, we have initially replicated biomarker panels that exclude acute rejection virtually $100 \%$ of the time on both HTG and NanoString assay platforms. A 6-mRNA panel on the NanoString assay was successfully validated on another set of unique, banked bio-samples from 126 patients, with 98\% NPV, 4\% PPV, 91\% sensitivity, and 15\% specificity. Heart transplant patients are biopsied as often as every week during the first 3 months post-transplant. Acute rejection, the most important cause of early morbidity and graft injury, occurs in $20-30 \%$ of patients during this timeframe. The BiT blood test has the potential to dramatically reduce the need for biopsies and aid clinicians in moderating immunosuppressive treatment according to individual patient needs.

\section{Session 3.6: Novel Therapies Targeting Cardiac Metabolism}

\section{3}

CARDIAC ENERGY METABOLIC PERTURBATIONS IN HEART FAILURE ASSOCIATED WITH OBESITY

GD Lopaschuk, S Sankaralingam, O Abo Alrob, CS Wagg, VB Patel, RS Padwal, DE Johnstone, AM Sharma, GY Oudit

Mazankowski Alberta Heart Institute, University of Alberta, Edmonton, Alberta

Dramatic alterations in cardiac energy metabolism occur in obesity and heart failure, including the development of cardiac insulin-resistance that can contribute to cardiac dysfunction. We therefore investigated what effect lowering body weight in obese mice with heart failure (by inducing caloric restriction, CR) had on cardiac function, and insulin-resistance. Obesity was induced in C57Bl/6 mice by feeding a high fat diet (HFD, 60\% kcal from fat) and heart failure was induced by a transverse aortic constriction (TAC). At 6 wk post-TAC, mice were then either continued on a HFD for a further $8 \mathrm{wk}$ period, or subjected to CR ( $40 \%$ decrease in calories). Control mice were fed a low fat diet (LFD, 12\% kcal from fat) or HFD and underwent a sham surgery. An increase in body weight was seen in HFD Sham mice $(45.2 \pm 2.1 \mathrm{~g})$ and HFD TAC mice $(42.1 \pm 1.3 \mathrm{~g})$ compared to LFD sham mice $(31.1 \pm 1.1 \mathrm{~g})$, as well as an impaired whole body glucose tolerance. CR of HFD TAC mice decreased body weight $(23.1 \pm 1.0 \mathrm{~g})$ and markedly improved whole body glucose tolerance. In comparison to HFD Sham, HFD TAC mice developed systolic dysfunction (\%EF, 47.8 \pm 3.0 vs. $25.2 \pm 2.8$, respectively, $\mathrm{P}<0.05$ ) and diastolic dysfunction (E'/A', $1.9 \pm 0.1$ vs. $1.1 \pm 0.1, P<0.05)$. CR significantly improved systolic (\%EF, 39.2 \pm 4.9 ), and diastolic (E'/A', 1.3 \pm 0.04 ) function. Compared to LFD Sham mice, HF TAC mice had a marked impairment of insulin-stimulated glucose oxidation in 
isolated working heart perfused at the end of the study. CR improved insulin-stimulated glucose oxidation compared to HFD TAC mice $(673 \pm 59$ vs $210 \pm 40 \mathrm{nmol} /$ joule, respectively $\mathrm{P}<0.05)$. This was associated with increased phosphorylation of Akt and decreased acetylation of pyruvate dehydrogenase (PDH activation). $\mathrm{CR}$ also decreased the high palmitate oxidation rates observed in the HFD TAC hearts $(206 \pm 25$ vs $677 \pm 128 \mathrm{nmol} /$ joule, respectively $\mathrm{P}<0.05)$, which was accompanied by a decrease in CD36 expression and a decreased acetylation of long chain acyl CoA dehydrogenase (LCAD inactivation). We conclude that weight loss in obese mice with heart failure improves cardiac insulin sensitivity, increases glucose oxidation, and improves cardiac function.

\section{4}

\section{ENERGY SUBSTRATE METABOLISM IN NORMAL AND} FAILING HEARTS

\section{FA Recchia}

Cardiovascular Research Center, Temple University School of Medicine, Philadelphia, Pennsylvania; Institute of Life Sciences, Scuola Superiore Sant'Anna, Piazza Martiri della Liberta, Pisa, Italy Under physiological conditions and at rest, the cardiac muscle generates most of its energy from the oxidation of long chain free fatty acids and, to a lesser extent, of lactic acid and glucose. Direct and indirect quantifications of cardiac substrate consumption have revealed profound alterations in fatty acids and carbohydrate utilization in pathological conditions such as ischemia, hypertrophy and failure. A characteristic metabolic change observed in severe heart failure is the reduced cardiac fatty acid oxidation associated with a marked increase in glucose oxidation. Whether this is an adaptive or a maladaptive mechanism remains an open question. We have tested the hypothesis that, in the failing heart, an abnormally high fraction of glucose is directed into the oxidative pentose phosphate pathway (oxPPP). While this pathway regenerates cytosolic NADPH necessary for the cellular anti-oxidant system, in the failing heart the excess NADPH, due to oxPPP upregulation, can also feed the superoxide-generating enzymes NADPH oxidase and uncoupled nitric oxide synthase. We provided indirect evidence, ex vivo, that the inhibition of oxPPP blunts superoxide production in homogenates of cardiac tissue harvested from failing hearts. Consistently, the acute administration of an oxPPP inhibitor in dogs with pacing-induced heart failure normalizes the high cardiac free radical production occurring in response to glycemic peaks. Although more evidence is needed to draw definitive conclusions, our findings suggest that pharmacological modulators of energy substrate metabolism might attenuate oxidative stress in the failing heart and prove therapeutically efficacious for the treatment of this syndrome.

\section{5}

MYOCARDIAL CELL MEMBRANE BIOKINETICS AND STRESS IONIC DISKINESY DISEASE CONTROL FOR CARDIOVASCULAR MORTALITY PREVENTION

\section{O Gomes}

ServCor-St Francis of Assisi Truth is Jesus Cardiovascular

Foundation, South America Section International Academy

Cardiovascular Sciences

In the myocardium the cell the membrane biokinetics is responsible for the EKG generation, being the $\mathrm{Na}$ ion flow the main responsible for the generation of the $\mathrm{R}$ wave. The ST segment is highly dependent of the transmembrane velocity of the Calcium flow and of its intracellular concentration. In patients with Stress Ionic Diskinesy Disease, more commonly during Stress Test, there is calcium transmembrane improved flow, responsible by the ST depression (STD) generally diagnosed as myocardium ischemia. This Exercise Induced Silent ST Segment Depression is a disease already proved to be related with high risk for Stroke and Cardiovascular Disease during life. Based on experimental study demonstrating the biokinetics specificity of myocardial cell membrane in EKG generation and the calcium-dependent variation of ST, 40 patients with STD, after a normal coronary circulation proven by tomographic myocardial perfusion during stress and rest, were treated with calcium inhibitor diltiazem (90 mg 8/8 hours) associated (20 cases) with a daily oral intake dose of $130 \mathrm{mg}$ of $\mathrm{Mg}$ chelate for 20 days. The repeated exercise stress test showed normal ST with improved physical and psychological fitness to stress supporting the involvement of the myocardium cell membrane biokinetis disease with calcium dependent myocardial ionic dyskinesia in the genesis of silent ST segment depression.

\section{Session 3.7: Non-Pharmacological Therapies for Heart Failure: Novel Devices}

\section{6}

\section{VAGAL STIMULATION IN HF: READY FOR PRIME TIME? ME Dunlap}

Director, HF Section, Heart and Vascular Center, MetroHealth Medical Center; Professor of Medicine, Physiology, \& Biophysics, Case Western Reserve University, Cleveland, Ohio, USA

Activation of the renin-angiotensin-aldosterone and sympathetic nervous systems are hallmarks of heart failure (HF), and continued activation of these systems portends a worse prognosis. Current effective HF therapy is aimed largely at blocking activation of these systems, including ACE inhibitors, beta blockers, and mineralocorticoid blockade. Parasympathetic influences are blunted markedly in HF, and decreased parasympathetic control portends a worse prognosis. In animal models of HF, enhancement of parasympathetic influences protects against ventricular arrhythmias and promotes beneficial LV anti-remodeling. Promising emerging technologies to enhance vagal influences on the heart chronically include baroreflex activation and vagal stimulation. This talk will explore mechanisms of abnormal parasympathetic control in HF along with data supporting vagal stimulation for HF, including recent and ongoing clinical trials in humans.

\section{7}

CARDIAC NEUROTRANSMISSION: SCIENTIFIC RATIONALE FOR TREATING VENTRICULAR TACHYCARDIA AND FIBRILLATION

\section{K Shivkumar \\ Cardiac Arrhythmia Center, UCLA Health System, Los Angeles, California, USA}

Cardiac neurotransmission (afferent and efferent) modulates key physiological functions of the heart (chronotropy, dromotropy, lusitropy and inotropy). Cardiac afferent information is relayed to four major destinations: (i) intrinsic cardiac nervous system, (ii) extracardiac-intrathoracic ganglia (e.g. stellate ganglion), (iii) spinal cord and (iv) to the brain stem (via the vagus). This information is processed and results in efferent cardiomotor control (via the sympathetic and parasympathetic nerves). This system ensures finetuned regulation of sympathetic-parasympathetic balance in the heart under normal and disease states. This system has also been shown to remodel (neuronal structure changes and trans-differentiation) in response to disease, and plays a major role in the pathophysiology and progression of heart disease (heart failure and arrhythmias leading to sudden cardiac death). Improved knowledge of the cellular and molecular processes governing cardiac innervation and the functional control of the myocardium in health and disease has resulted in a logical mechanistic framework for developing neuraxial therapies for preventing SCD and other arrhythmias.

\section{8}

\section{REGENERATIVE CELL THERAPY FOR HEART FAILURE}

MJB Kutryk

Division of Cardiology, Keenan Research Center for Biomedical Science at the Li Ka Shing Knowledge Institute, St Michael's Hospital, University of Toronto, Toronto, Ontario

While improvements in medical and interventional therapies for ischemic heart disease have reduced early mortality, they have resulted in a higher incidence of chronic heart failure among survivors. Recurrent hospitalizations and premature death, prevalent in this growing patient population, have imposed a major unmet need associated with the inability of current, largely palliative, therapies to address tissue destruction post-infarction. 
Stem cell-based regenerative treatment has been proposed as a promising approach for the treatment of both ischemic and non-ischemic cardiomyopathies based on numerous animal studies. A variety of potential stem cell types, including skeletal myoblasts and bone marrow and circulating stem/ progenitor cells, have been investigated in clinical trials for cardiac repair and regeneration, and have demonstrated their ability to improve cardiac function, infarct size, and cardiac remodeling. Based on these exciting but tentative results, other stem cell types are being explored for their particular advantages as a source of adult stem cells. The results of the clinical trials of cell therapy for heart failure will be reviewed and the proposed mechanisms of benefit discussed. The design and promise of ongoing trials will be described.

\section{Session 3.8: The James Willerson Young Investigator Competition in Cardiovascular Research}

\section{9}

\section{ROLE OF BONE MARROW AND NEUROINFLAMMATION IN HYPERTENSION}

MM Santisteban ${ }^{1}$, N Ahmari ${ }^{2}$, JM Carvajal ${ }^{1}$, MB Zingler ${ }^{1}, \mathrm{Y} \mathrm{Qi}^{3}$, S Kim $^{1}$, J Joseph ${ }^{1}$, F Garcia-Pereira ${ }^{2}$, RD Johnson ${ }^{2}$, V Shenoy ${ }^{4}$, MK Raizada ${ }^{1}$, J Zubcevic ${ }^{2}$

${ }^{1}$ Department of Physiology and Functional Genomics, College of Medicine; ${ }^{2}$ Department of Physiological Sciences, College of Veterinary Medicine; ${ }^{3}$ Division of Cardiovascular Medicine, Department of Medicine; ${ }^{4}$ Department of Pharmacodynamics, College of Pharmacy, University of Florida, Gainesville, Florida, USA Microglial activation and dysfunctional autonomic nervous system (ANS) activity are associated with hypertension (HTN). Despite evidence that impaired sympathetic nerve activity supplying the bone marrow (BM) increases inflammatory cells and decreases angiogenic cells, little is known about the reciprocal impact of BM-derived inflammatory cells on neuroinflammation and HTN. The overall objective of this study was to investigate the hypothesis that the $\mathrm{BM}$ cells from hypertensive animals are proinflammatory, contribute to neuroinflammation and thus important in sustained HTN. Chimeric SHRs were generated by reconstitution with the BM from the WKY rats. The resultant chimeric SHR displayed significant reduction in mean arterial pressure (MAP; SHR-WKY $138 \pm 11 \mathrm{mmHg}$ vs SHR-SHR $188 \pm 6 \mathrm{mmHg}$ ) associated with attenuation of both central and peripheral inflammation compared with SHR-SHR. In contrast, an elevated MAP (WKY-SHR $147 \pm 16 \mathrm{mmHg}$ vs WKY-WKY $114 \pm 2 \mathrm{mmHg}$ ) along with increased central and peripheral inflammation was observed in chimeric WKY rats reconstituted with SHR BM. Minocycline, an inhibitor of microglia activation, attenuated HTN in both SHR and chronic angiotensin II (Ang II)-infused rats. This was accompanied by decreased sympathetic drive and peripheral inflammation. Furthermore, in chronic Ang II-infused rats, minocycline reduced the number of BM-derived cells in the PVN. Therefore, the BM contributes to HTN by increasing peripheral inflammatory cells and their extravasation into the brain. Minocycline effectively modified peripheral and neurogenic components of HTN. Taken together, these observations support the hypothesis that dysfunctional brain-BM activity is important in HTN, and targeting this axis may be an innovative strategy for neurogenic resistant HTN therapy.

This study was supported by National Institutes of Health grant HL33610 (M.K.R.), American Heart Association Pre-doctoral Fellowship 14PRE18590018 (M.M.S.), and AHA Scientist Development Grant 14SDG18000010 (J.Z.).

\section{0}

ATRIAL FIBRILLATION DRIVEN BY MICRO-ANATOMIC INTRAMURAL RE-ENTRY REVEALED BY SIMULTANEOUS SUB-EPICARDIAL AND SUB-ENDOCARDIAL OPTICAL MAPPING IN EXPLANTED HUMAN HEARTS

B] Hansen ${ }^{1}$, J Zhao ${ }^{2}$, TA Csepe ${ }^{1}$, BT Moore ${ }^{1}$, N Li ${ }^{1}$, LA Jayne ${ }^{1}$, A Kalyanasundaram ${ }^{1}$, P Lim ${ }^{2}$, A Bratasz ${ }^{3}$, KA Powell ${ }^{3}$, O Simonetti, ${ }^{3,4}$, RSD Higgins $^{3,5}$, A Kilic ${ }^{3,5}$, PJ Mohler ${ }^{1,3,4}$, PML Janssen ${ }^{1,3,4}$, R Weiss ${ }^{3,4}$, JD Hummel ${ }^{3,4}$, VV Fedorov ${ }^{1,3}$

${ }^{1}$ Department of Physiology and Cell Biology, The Ohio State University Wexner Medical Center, Columbus, Ohio, USA; ${ }^{2}$ Auckland Bioengineering Institute, The University of Auckland, Auckland, New Zealand; ${ }^{3}$ Davis Heart and Lung Research Institute; ${ }^{4}$ Department of Internal Medicine; ${ }^{5}$ Department of Surgery, The Ohio State University Wexner Medical Center, Columbus, Ohio, USA

AIMS: The complex architecture of the human atria may create physical substrates for sustained reentry to drive atrial fibrillation (AF). The existence of sustained, anatomically-defined AF drivers in humans has been challenged partly due to the lack of simultaneous endocardial/epicardial (Endo/Epi) mapping coupled with high-resolution 3D structural imaging. METHODS AND RESULTS: Coronary-perfused human right atria from explanted diseased hearts ( $n=8,43-72$ y.o.) were optically mapped simultaneously by three high-resolution CMOS cameras (two aligned Endo/ Epi views $\left(330 \mu \mathrm{m}^{2}\right.$ resolution) and one panoramic view). 3D Gadoliniumenhanced MRI (GE-MRI, $80 \mu \mathrm{m}^{3}$ resolution) revealed the atrial wall structure varied in thickness $(1.0 \pm 0.7 \mathrm{~mm}-6.8 \pm 2.4 \mathrm{~mm})$, transmural fiber angle differences, and interstitial fibrosis causing transmural activation delay from $23 \pm 11 \mathrm{~ms}$ to $43 \pm 22 \mathrm{~ms}$ at increased pacing rates. Dual-sided sub-Endo/sub-Epi optical mapping revealed that AF was driven by spatially and temporally stable intramural reentry with $107 \pm 50 \mathrm{~ms}$ cycle length and transmural activation delay of up to $67 \pm 31 \mathrm{~ms}$. Intramural reentrant drivers were captured primarily by sub-Endo mapping, while sub-Epi mapping visualized reentry or "breakthrough" patterns. Reentrant drivers were anchored on 3D microanatomic tracks $\left(15.4 \pm 2.2 \times 6.0 \pm 2.3 \mathrm{~mm}^{2}\right.$, $2.9 \pm 0.9 \mathrm{~mm}$ depth) formed by atrial musculature characterized by increased transmural fiber angle differences and interstitial fibrosis. Subsequent targeted radiofrequency ablation of the tracks terminated 8/8 AF drivers leaving AF uninducable.

CONCLUSIONS: Integrated 3D structural-functional mapping of the diseased human heart revealed that the complex atrial microstructure caused significant differences between Endo vs Epi activation during pacing and sustained AF driven by intramural reentry anchored to fibrosisinsulated atrial bundles. Radiofrequency ablation of microanatomic reentry tracks represents a promising treatment of AF.

\section{1}

\section{TLR-4 DEPENDENT COLLAGEN LOSS IN HUMAN CAROTID PLAQUE SMOOTH MUSCLE CELLS}

V Rai $^{1}$, VH Rao ${ }^{2}$, S Stoupa ${ }^{2}$, DK Agrawal ${ }^{1,2}$

${ }^{1}$ Center for Clinical and Translational Science; ${ }^{2}$ Department of Biomedical Sciences, Creighton University, Omaha, Nebraska, USA Atherosclerosis is a multifactorial chronic inflammatory disease resulting in development of atheroma and plaque. Thromboembolism may result from rupture of thin fibrous cap and subsequent ischemic stroke. The precise mechanism of plaque rupture remains to be defined.

Triggering receptor expressed on myeloid cells-1 (TREM-1) expressed on immune cells amplify inflammation. Toll-like receptors (TLRs) induced by stimulation of pattern recognition receptors are involved in the pathogenesis of various cardiovascular diseases. However the role of TLR-4 in the destabilization of carotid plaques in atherosclerosis remains unknown. The mRNA expression of TREM-1 and TLR-4 by qPCR were increased significantly in symptomatic (S) compared to asymptomatic (AS) patients with carotid stenosis in both tissue extracts and isolated vascular smooth muscle cells (VSMCs). The TREM-1 and TLR-4 immunofluorescence was greater in tissue sections of $\mathrm{S}$ compared to AS plaques which confirmed our results of qPCR. Increased MMP-1 and -9 and decreased collagen I and III mRNA transcripts were observed in VSMCs from S compared to AS patients with 
carotid stenosis. Inhibitors of NF-kB decreased the expression of TREM-1, TLR-4 and MMP-9 and increased mRNA transcripts of collagen type I and III in VSMCs of S compared to AS patients with carotid stenosis. Results of this study suggest the role of TREM-1 and TLR-4 in the pathophysiology and stability of atheroma in carotid stenosis. Collectively, these data demonstrate that selective blockade of TLR-4 may contribute to the development of new therapies and promising targets for stabilizing atherosclerotic plaques.

This work was supported by the National Institutes of Health RO1 HL073349 to DKA

\section{2 \\ MICRORNA-21 UP-REGULATION IN THE FAILING RIGHT VENTRICLE}

LC Powers ${ }^{1}$, M Seki ${ }^{1}$, G Chacko ${ }^{1}$, S Parikh ${ }^{1}$, T Wang, A Rudman¹, A Sabri ${ }^{1}$, EJ Tsai ${ }^{2}$, FA Recchia ${ }^{1}$

${ }^{1}$ Temple University School of Medicine, Philadelphia, Pennsylvania; ${ }^{2}$ Columbia University College of Physicians and Surgeons,

New York City, New York, USA

microRNA (miR) dysregulation has been implicated in several cardiovascular pathologies. We hypothesized that altered miR expression specific to the failing right ventricle (RV) plays a role in RV dysfunction. Seven dogs were subjected to 4-week tachypacing to induce congestive heart failure (HF). Seven non-paced dogs were used as controls. Functional assessments confirmed the development of RV dysfunction and secondary pulmonary hypertension in HF vs control: RV end-diastolic pressure was $11.6 \pm 1.7$ vs $5.62 \pm 1.3 \mathrm{mmHg}$; mean pulmonary artery pressure was $29.7 \pm 2$ vs $21.2 \pm 1.1$ $\mathrm{mmHg}$; tricuspid annular plane systolic excursion was $10.1 \pm 2.4$ vs $15.8 \pm 1.3 \mathrm{~mm}$; RV fractional area change was $0.22 \pm 0.02$ vs $0.50 \pm 0.02$ (all $\mathrm{P}<0.05$ ). miR microarray analysis showed upregulation of several miRs in HF-RV vs HF-LV and control myocardium. We focused on miR-21, which increased four-fold in HF-RV vs control RV (with no change in HF-LV vs control LV) and is known to target phosphatase and tensin homolog (PTEN), a negative regulator of fibroblast proliferation and of Akt activation/phosphorylation. Myocardial PTEN was indeed selectively downregulated in HF-RV and not in HF-LV, consistent with increased fibrosis in $(10.73 \pm 0.19 \%$ vs $4.84 \pm 0.17 \%, P<0.05)$ and activation of Akt phosphorylation, a positive regulator of fibroblast proliferation. To further test the cell-specificity of miR-21-mediated effects, we cultured neonatal rat cardiomyocytes and fibroblasts in the presence of miR-21 or scrambled miR. No significant changes were found in cardiomyocytes, while PTEN was downregulated in miR-21-treated fibroblasts. These findings suggest that miR-21 upregulation is a novel major determinant of fibrosis, and thereby wall stiffening and functional impairment, in the failing RV.

Acknowledgements: This project is funded by a grant from the National Institutes of Health.

\section{3}

COMBINATION TREATMENT WITH TADALAFIL AND
HYDROXYCHLOROQUINE IMPROVES CIRCULATING
INSULIN, IGF1 AND REDUCES BLOOD TRIGLYCERIDES
WITH CONCOMITANT INCREASED EXPRESSION OF
CARDIAC SIRT3 PROTEIN IN TYPE 2 DIABETIC MICE

R Wang, L Xi, C Kukreja

Division of Cardiology, Department of Internal Medicine, Virginia

Commonwealth University, Richmond, Virginia, USA

INTRODUCTION: Increased prevalence of cardiovascular diseases is associated with risk factors (e.g. hyperlipidemia, insulin resistance) in Type 2 diabetes patients. We hypothesized that combination treatment with phosphodiesterase type 5 inhibitor Tadalafil (TAD) and anti-malaria/anti-inflammatory drug hydroxychloroquine (HCQ) could provide synergetic beneficial effects in reduction of cardiovascular risk factors in Type 2 diabetic mice. METHODS: Adult male db/db mice were randomly assigned into 4 treatment groups ( $\mathrm{n}=7-9)$ and oral-gavaged daily for 7 days: 1) Control (vehicle), 2) TAD (6mg/kg), 3) HCQ (50mg/kg) and 4) TAD+HCQ. Blood glucose, insulin, insulin-like growth factor-1 (IGF-1) and triglycerides levels were measured. Cardiac SIRT3 protein expression was determined with western blot.
RESULTS AND CONCLUSION: Compared with Control, TAD-treated animals had lower blood glucose level $(198 \pm 30$ vs $365 \pm 44 \mathrm{mg} / \mathrm{dL}, \mathrm{p}<0.05)$. Reduced blood glucose level was also seen in HCQ and TAD+HCQ groups. Increased plasma insulin level was observed in both HCQ and TAD+HCQ groups with more synergistic increase following the combination treatment (HCQ $403 \pm 54$ and TAD+HCQ $523 \pm 73$ vs. Control $232 \pm 30 \mathrm{mU} / \mathrm{L}$, $\mathrm{p}<0.05)$. Increased IGF- 1 and decreased triglycerides levels were found only in TAD+HCQ group. Cardiac SIRT3 protein expression was upregulated in both HCQ and TAD+HCQ groups. These results suggest that TAD+HCQ treatment causes improvement in overall metabolic profile in Type 2 diabetic mice. Furthermore, increased Sirt3 expression may protect cardiac mitochondria through suppression of oxidative stress. The data suggest that TAD + HCQ could be a novel combination therapy for reducing cardiovascular risk factors in Type 2 diabetes.

Acknowledgment: This study is supported by National Institutes of Health (1RO1HL118808).

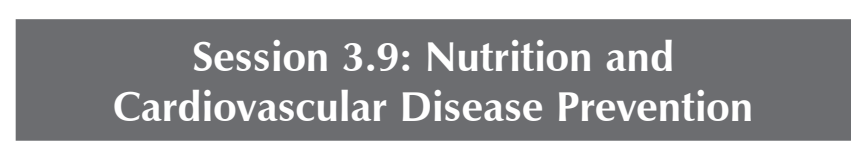

\section{4}

\section{EVALUATION OF ANTI DIABETIC ACTIVITY OF HYDROALCOHOLIC EXTRACT OF BARK OF PROSOPIS CINERARIA AGAINST STREPTOZOTOCIN INDUCED DIABETES IN RATS}

\section{SS Agrawal, A Gupta, G Sharma}

Amity Institute of Pharmacy, Amity University Sec-125, Noida, Uttar Pradesh, India

Diabetes mellitus is a major public health hazard prevailing all over the globe. The global increase in the prevalence of diabetes is due to aging, obesity and lack of physical inactivity. The burden of diabetes is increasing globally, particularly in developing countries. 347 million people worldwide suffer diabetes. In 2004, an estimated 3.4 million people died as a consequence of high fasting blood sugar. More than $80 \%$ of diabetes deaths occured in low- and middle-income countries. WHO projects that diabetes will be the 7th leading cause of death in 2030. Healthy diet, regular physical activity, maintaining a normal body weight and avoiding tobacco use can prevent or delay the onset of type 2 diabetes.

In the present studies, the role of hydroalcoholic extract of bark of Prosopis cineraria (khejri) in reducing the Blood Glucose level in Streptozotocin (60 mg/kg i.p.) $40 \mathrm{mg} / \mathrm{kg}$ IP respectively \& XZ induced diabetes in albino wistar rats were conducted. Thirty six male albino rats were divided into six groups of six animals each. Group I served as control, Group II received streptozotocin, Group III received glimepiride $(2 \mathrm{mg} / \mathrm{kg})$, Group IV, V and VI are treated with crude hydroalcoholic extract of Prosopis cineraria bark doses of 200,400 and $600 \mathrm{mg} / \mathrm{kg}$ body weight daily for 30 days. Blood glucose was estimated on day $1,4,7,14,21$ and 28 which was compared with the standard. Other biochemical parameters estimated included total cholesterol, triglyceride, HDL, VLDL and LDL levels.

It was observed that there was decrease in weight of diabetic rats while increase in rats treated with the test drug. Blood glucose level was decreased by $18 \%$ in $200 \mathrm{mg} / \mathrm{kg}$ extract dose, $27 \%$ in $400 \mathrm{mg} / \mathrm{kg}$ dose and $29 \%$ in $600 \mathrm{mg} / \mathrm{kg}$ dose as compared to standard which expected $42 \%$ decrease in blood glucose level. Triglycerides, cholesterol, LDL and VLDL level were decreased in test drug treated animals as compared to diabetic untreated rats while there was increase in HDL level. The results were significantly different $(\mathrm{P}<0.05)$. 
075

WHAT PREVENTIVE STRATEGIES CAN WE USE TO CURB THE ESCALATING MORTALITY AND MORBIDITY ASSOCIATED WITH CARDIOVASCULAR DISEASES?

HS Buttar

Department of Pathology and Laboratory Medicine, Faculty of Medicine, University of Ottawa, Ottawa, Ontario

The burdens of premature mortality and morbidity due to non-communicable diseases such as cardiovascular diseases (CVDs), obesity, diabetes mellitus, cancers, musculoskeletal disorders, anemia, and depression are escalating worldwide. Though these diseases generally manifest in middle age and beyond, it is now recognised that roots of these diseases lie in childhood and adolescence. The conventional risk factors of CVD consist of hypertension, hyperlipidemia, atherosclerosis, and hyperglycemia. Lifestyle factors including tobacco use, lack of exercise, unhealthy dietary habits, and low socioeconomic status contribute heavily to the development of obesity, diabetes and CVD in children and adults. Sugar-loaded beverages and excessively salted foods are also potential risk factors. Diets rich in whole grains, fruits and vegetables, olive oil, fish, low-fat dairy products, and moderate wine consumption are linked with lower incidence of CVD. Lifestyle modifications such as regular physical activity (about 30 $\mathrm{min} /$ day), restriction of caloric and sodium intake, smoking cessation and moderate alcohol consumption are recommended for improving cardiovascular health and quality of life. Ingestion of phytosterol-enriched foods, vitamins, minerals, and amino acids assist to improve overall health beyond basic nutritional functions. Emerging evidence suggests that dietary supplements containing flavonoids and antioxidants modulate gene and protein expression and thereby modify endogenous metabolic pathways and homeostasis, and consequently reduce the risk of CVD and chronic diseases multifactorial in origin. Given the scope and prevalence of CVDs, a cost effective population health strategy - 'prevention is better than cure' - would be the most appropriate model to adopt to deal with CVD-related mortality and morbidity.

\section{6}

\section{LYSOPHOSPHATIDIC ACID, GROWTH FACTORS, AND MATRICELLULAR PROTEINS IN VASCULAR SMOOTH MUSCLE CELL MIGRATION}

\section{F Zhang, D Wu, F Hao, X Xu, M-Z Cui}

University of Tennessee College of Veterinary Medicine, Knoxville, Tennessee, USA

Vascular smooth muscle cell (SMC) migration from the tunica media to the intima is a key component of neointimal formation following vascular injury and atherosclerotic lesion formation. Lysophosphatidic acid (LPA), a potent bioactive lipid found in atherosclerotic lesions and produced by activated platelets, has been shown to markedly induce SMC migration. Platelet-derived growth factor (PDGF) is a major growth factor involved in SMC migration. We have recently identified matricellular protein Cyr61 (CCN1) to be a key extracellular mediator in LPA- and PDGF-induced SMC migration. LPA and PDGF induced temporal and spatial expression of Cyr61, which promptly accumulated in the cellular Golgi apparatus and then translocated to the extracellular matrix. Cyr61 antibody blockade and siRNA inhibition both diminished LPA- or PDGF-induced SMC migration, indicating a novel regulatory role of Cyr61. SMCs derived from LPA receptor 1 (LPA1) knockout mice lack the ability of Cyr61 induction and cell migration, supporting the concept that LPA1 is required for Cyr61 expression and migration. Our data show that the newly synthesized Cyr61 physically interacted with the SMC plasma membrane integrins a6 $\beta 1$ and $\alpha v \beta 3$. We further demonstrated that Cyr61 and integrins are integral and important components of the LPA and PDGF signaling pathways via an "outside-in" signaling route to activate intracellular focal adhesion kinase (FAK), leading to cell migration. Therefore, extracellular Cyr61 convergence with lipid/growth factor signaling and integrin/FAK signaling is a new concept of lipid/growth factor-induced cell migration. These data provide new insights into mechanisms underlying SMC migration-related disorders, including atherosclerosis and restenosis.

\section{7}

\section{BENEFICIAL ROLE OF OMEGA-3 FATTY ACIDS IN CVD: WHY ARE THERE CONTROVERSIES IN CLINICAL TRIALS' OUTCOME? \\ NA Shaikh}

University of Toronto and Pivotal Therapeutics Inc, Toronto, Ontario The role of omega-3 polyunsaturated fatty acids (omega-3 PUFAs) for the prevention of cardiovascular disease (CVD) has been known for many decades. Although some clinical trials support the epidemiological lines of evidence that omega-3 PUFAs protect against coronary heart disease, all cause or cardiac mortality, many others have drawn no such conclusions. This presentation provides a prospective that could explain some of the inconsistencies in trial outcomes. This viewpoint is not meant to address all the differences or cover all studies that could contribute to the variability in the findings, but to point out some of the factors that could affect trial results and meta-analyses outcomes. Meta-analysis of the RCTs are more prone to be affected adversely by the inclusion of studies with heterogeneous patients with mixed end points than those conducted with homogeneous group. Other factors that could contribute to the variability of the outcome are the use of different omega-3 PUFA formulations and doses, choice of placebo, lack of pre- and post omega-3 PUFA measurements as well as differential uptake of omega-3 PUFAs in study subjects. Consideration of these factors may play an important role in subsequent trial designs for the consolidation of outcome measures and efficacy of omega-3 PUFAs in primary and secondary prevention of CVD.

Session 3.10: Instability of
Atherosclerotic Plaque: Underlying
Mechanisms and Biomarkers

\section{8}

\section{ENDOTHELINS IN CARDIOVASCULAR DISEASES: ESTABLISHED AND EMERGING TARGETS}

\section{P D'Orléans-Juste, M Houde, L Desbiens}

Institute of Pharmacology, Université de Sherbrooke, Sherbrooke, Quebec

In the past 28 years, over 28,000 studies have been published in the field of endothelins (ET). Albeit thousands of primary pulmonary hypertensive patients are currently treated with ET antagonists such as TRACLEER ${ }$ (Bosentan), LETAIRIS® (Ambrisentan) or OPSUMIT® (Macitentan), clinical trials with these blockers in several other types of cardiovascular diseases, have been disappointing. This presentation provides an update on the currently pursued targets in the field of endothelins based on observations to be presented at the 14th International Conference on Endothelins (Savannah, Ga, September 2-5th, 2015). The neurovascular role of ET-1 in degenerative diseases such as Multiple Sclerosis will be discussed. In addition new roles for ET receptors in essential hypertension and acute renal failure will be presented. On the other hand, the role for emerging ET-1 synthesis pathway in mouse models of acute cardiac infarct or early atherosclerosis will be discussed. Finally, the intriguing pharmacodymanic properties of the potent vasoactive peptide in a freely moving mouse model will be presented.

Overall, this presentation will summarize several promising avenues even after almost 30 years of intensive research in the field of endothelins. This work is supported by the Canadian Institutes for Health Research (CIHR).

\section{9}

NOVEL ROLES FOR FORMIN DRF1 IN MYOCARDIAL STRESS

R Ramasamy

Langone Medical Center, New York University, New York City, New York, USA

Myocardial ischemia-reperfusion (I/R) injury is a major cause for heart failure and sudden death. Our recent studies show that after myocardial I/R in mice, expression of diaphanous formin related 1 (Drf1) was increased in the heart leading to enhanced tissue injury and deterioration of myocardial 
function. Genetic deletion of Drf1 limited myocardial I/R injury and improved heart function. These effects were recapitulated in the hypoxia/ reperfused $\mathrm{H} 9 \mathrm{C} 2$ rat cardiomyocytes. Notably, we show that the reduction in injury can be attributed to improved calcium handling and actin-related transcriptional changes involving early growth response- 1 and serum response factor. Our work demonstrates a novel formin driven mechanism mediating myocardial $\mathrm{I} / \mathrm{R}$ injury and identifies new therapeutic target for acute myocardial infarction.

\section{Session 3.11: Echocardiography and Other Novel Modalities}

\section{0}

\section{DIABETES INDUCED CARDIOMYOPATHY (DCM)}

GE Nagib El-kilany

Consultant of Cardiology, United Arab Emirates

Diabetic cardiomyopathy is a distinctly primary disease process, independent of coronary artery disease, which leads to heart failure in diabetic patients. The link between heart failure (HF) and diabetes is well documented, but the existence of diabetic cardiomyopathy as a distinct clinical entity continues to be the subject of debate. Rubler et al. coined the term 'diabetic cardiomyopathy' after performing post mortem studies in diabetic patients with cardiac failure having excluded alcohol, hypertension, coronary and structural heart disease as possible etiologies. In diabetes induced cardiomyopathy, identification of high-risk patients in the presymptomatic phase and those prone to sudden cardiac death has become the "holy grail" for physicians and efforts have evolved for risk profiling of such patients population. Currently detailed novel echocardiographic examination and measurements of NT-pro BNP levels provide sensitive methods that identify the early signs of myocardial dysfunction among diabetic patients. Emerging novel diagnostic techniques such as tissue Doppler imaging (TDI) and deformation imaging - to identify patients earlier, together with the development of more targeted therapeutic strategies; may lead to improved outcome in patients with DCM.

\section{1}

\section{COMPUTED TOMOGRAPHY CORONARY ANGIOGRAPHY: DIAGNOSTIC AND PROGNOSTIC VALUE}

\section{FB Sozzi}

Cardiology Unit, Policlinico Hospital, Milan, Italy

Coronary computed tomography (CCT) is increasingly being used as a tool for non-invasive visualization of the coronary arteries. The technique provides informations about atherosclerotic plaque burden and plaque composition. The diagnostic and prognostic value of CCT is high. Its accuracy needs to be assessed in management outcome studies, in which diagnostic and therapeutic strategies are decided based on CCT alone, without reference to coronary angiography results. A main point is that plaque composition represents a long-term predictor of cardiac events. In a follow-up study on the predictive value of CCT, Sozzi et al (1) demonstrated that noncalcified and mixed plaques carried a worse prognosis compared to calcified plaques. Referring to plaque vulnerability concept, Mann et al (2) found that lipid core size and minimal cup thickness, two major determinants of plaques vulnerability, were not related to the absolute plaque size or degree of stenosis, in patients who died suddenly of coronary artery disease. Accordingly, atherosclerotic plaque growth and destabilization are highly variable. Many angiographic studies have demonstrated that most of the acute myocardial infarction are characterized by the occlusion of coronary arteries without previous significant stenosis. Thus, plaque progression and clinical outcome are not always closely related. Both factors are not easily predictable on exclusively clinical and angiographic grounds.

REFERENCES:

1. Sozzi FB, et al. Long-term follow-up of patients with first-time chest pain having 64-slice computed tomography. AJC 2011;15:516-21.

2. Mann JM, et al. Vulnerable plaque. Relation of characteristics to degree of stenosis in human coronary arteries. Circulation 1996;94:928-931.

\section{2}

\section{THE ROLE OF ECHOCARDIOGRAPHY IN DIAGNOSING EARLY CHANGES FOLLOWING CHEMOTHERAPY IN BREAST CANCER DISEASES}

\section{K Hristova}

\section{University National Heart Hospital, Sofia, Bulgaria}

The literature exploring the utility of advanced echocardiographic techniques (such as deformation imaging) in the diagnosis and prognostication of patients receiving potentially cardiotoxic cancer therapy has involved relatively small trials in the research setting. In this systematic review of the current literature, we describe echocardiographic myocardial deformation parameters in 2500 patients during or after cancer chemotherapy for 3 clinically-relevant scenarios. All studies of early myocardial changes with chemotherapy demonstrate that alterations of myocardial deformation precede significant change in left ventricular ejection fraction (LVEF). Using tissue Doppler-based strain imaging, peak systolic longitudinal strain rate has most consistently detected early myocardial changes during therapy, whereas with speckle tracking echocardiography (STE), peak systolic global longitudinal strain (GLS) appears to be the best measure. A 10\% to $15 \%$ early reduction in GLS by STE during therapy appears to be the most useful parameter for the prediction of cardiotoxicity, defined as a drop in LVEF or heart failure. In late survivors of cancer, measures of global radial and circumferential strain are consistently abnormal, even in the context of normal LVEF, but their clinical value in predicting subsequent ventricular dysfunction or heart failure has not been explored. Thus, this systematic review confirms the value of echocardiographic myocardial deformation parameters for the early detection of myocardial changes and prediction of cardiotoxicity in patients receiving cancer therapy.

\section{3 \\ NON-INVASIVE IMAGING FOR THE ASSESSMENT OF RIGHT VENTRICULAR STRUCTURE AND PERFORMANCE \\ S Kutty \\ Department of Cellular \& Integrative Physiology, University of Nebraska Medical Center, Omaha, Nebraska, USA}

The right ventricle plays an important role in various forms of heart disease. The assessment of right ventricular structure and function is an oftenencountered clinical challenge due to the chamber's complex geometry. Right ventricular assessment has assumed a lot of focus recently because it is an independent prognostic indicator of morbidity and mortality in heart failure and pulmonary hypertension. Echocardiography is the primary modality used for right heart imaging, and cardiovascular magnetic resonance is the current reference standard. Non-invasive imaging has shed light on the right ventricle's adaptation to pressure and volume overload states. The goal of this presentation is to review non-invasive imaging in the assessment of the right heart, focusing on newer two and three-dimensional echocardiography techniques, cardiovascular magnetic resonance, and deformation imaging. Improvements in non-invasive imaging will lead to a better understanding of the pathophysiology of right heart failure, and enhanced ability to follow responses to treatment. 
Session 3.12: Jawahar Mehta Young Faculty Orations

\section{4}

\section{A NEW METHOD FOR MEASURING THE FUNCTIONAL CARDIOMYOCYTES CONTRACTILITY AND DRUGS EVALUATION}

J Rajasingh ${ }^{1}$, S Rajasingh ${ }^{1}$, A Czirok ${ }^{2}$, S Samanta ${ }^{1}$, DG Isai ${ }^{2}$, E Kosa ${ }^{2}$, B Dawn ${ }^{1}$

${ }^{1}$ Cardiovascular Research Institute, Division of Cardiovascular Diseases, Department of Internal Medicine; ${ }^{2}$ Department of Anatomy and Cell Biology, University of Kansas Medical Center, Kansas City, Kansas, USA

INTRODUCTION: Current methods of assessing the functional property of cardiomyocytes (CMCs), such as patch clamping or dye-based confocal calcium imaging, are labor-intensive, invasive, which can affect the cells' contractility. No optimal method is currently available to assess functional CMCs.

HYPOTHESIS: We hypothesized that a novel particle image velocimetry (PIV) method would provide accurate assessment of CMC contractility, maturity and drug effects.

METHODS: Recently, we have reported an efficient animal-free and viral-free method of generating iPSC from human somatic cells. These iPSCs were further differentiated into functional CMCs with defined culture conditions.

RESULTS: To analyze the contractility of iPSC-derived CMCs (iCMCs), we recorded the same areas of culture plate at different time-points using high frame-rate video microscopy. Our novel image analysis technique provided beat patterns time-series data of cell displacement, measured relative to a resting reference state. As beat patterns of recordings in video microscopic images revealed, the contractility of early CMC nodes was asynchronous in space and irregular in time. During subsequent days after the onset of beating, however, the spatially distinct contractile centers became synchronous and gradual increased in beating frequency. Our PIV method in agreement with calcium imaging showed that the CMCs treated with verapamil or isoproterenol changes both the amplitude and beating frequency.

CONCLUSIONS: This PIV image analysis is a novel method that enables assessment of CMC contractility multiple times, if necessary, without jeopardizing the biology of cells. This method may also prove beneficial for drug screening and detection of cardiotoxicity in iPSC-derived CMCs.

\section{5}

\section{EFFEROCYTOSIS HEALS THE HEART: NEW INFLAMMATORY} TARGETS FOR MYOCARDIAL REPAIR

\section{E Thorp}

Department of Pathology and Feinberg Cardiovascular Research Institute, Northwestern University Medical School, Chicago, Illinois, USA

Heart failure after myocardial inflammation or myocardial infarction (MI) is a significant cause of morbidity and mortality. Though pharmacological advances have significantly reduced mortality, the residual risk of post MI-induced heart failure is increasing. This necessitates development of new approaches to preserve heart function. Post infarction, we have discovered that efficient phagocytic removal of dying cardiomyocytes by efferocytosis is critical to initiating resolving inflammation and cardiac repair. Myocardial efferocytosis is in part driven by the apoptotic cell receptor and tyrosine kinase MERTK. Our findings in humans suggest MERTK is inactivated during MI by proteolysis, implicating natural inefficiencies in cardiac repair. Collaborative efforts to recombinantly inhibit the susceptibility of MERTK to proteolytic inactivation in vivo reveal enhanced cardiac repair in preliminary data. Current studies also show that suppression of anti-phagocytic ligands on the surface of cardiomyocytes, such as CD47, enhance engulfment by phagocytes. These data implicate cross talk between macrophages and cardiomyocytes as a potential therapeutic target for cardiac healing. Several ongoing lines of investigation include (I) the degree to which MERTK-dependent efferocytosis and proteolysis drives the extent of post MI repair in the setting of risk factors such as hyperlipidemia and clinically-relevant reperfusion, (II) MERTK-dependent and independent mechanisms of efferocytosis and inflammation resolution during hypoxia, and (III) novel cardiomyocyte interactions with macrophages.

\section{6}

\section{REGULATION OF CARDIAC GENE EXPRESSION BY MED1}

\section{K Spitler, DD Hall, CE Grueter}

\section{University of lowa, lowa City, lowa, USA}

Despite significant advances in cardiovascular research, the complex regulatory mechanisms that control cardiac gene expression in response to developmental and environmental stimuli are not well defined. Thus, there is a major need for new insights into the mechanisms that govern transcriptional regulation of developmental processes in the heart as a prelude to the design of therapeutic strategies to normalize cardiac function during disease. Common developmental pathologies in the heart leading to decreased cardiac function include mitochondrial dysfunction and disrupted cardiac energetics. Med1, a key component of the Mediator complex, functions as a transcriptional cofactor involved in regulating mitochondrial gene expression and metabolism. Multiple studies have demonstrated that systemic deletion of Med1 is embryonic lethal due to cardiac defects. However, the mechanism by which Med1 functions to regulate cardiac development and disease has not been examined. To test the hypothesis that Med1 governs a gene network that is critical for the heart to respond to developmental and pathological signaling events, we generated a mouse model with a cardiac specific knockout of Med1 (cMED1KO). Postnatal deletion of Med1 in cardiomyocytes is lethal by 3-6 weeks of age due to heart failure. We performed RNA-seq analysis and identified multiple key pathways were augmented including down regulation of metabolic and cation transport pathways and up regulation of developmental and cell cycle processes. Further studies are underway to identify the molecular targets of Med1 that are necessary for normal cardiac response to developmental and environmental signals regulating transcriptional remodeling in the heart.

ACKNOWLEDGEMENT: The authors acknowledge JK Reddy for the MED1 fllfl mouse.

\section{7}

VITAMIN D REGULATES GLUCOSE METABOLISM THROUGH TARGETING FOXO1 GENE IN SKELETAL MUSCLE

\section{$\underline{\text { S Chen, DK Agrawal }}$}

Center for Clinical \& Translational Science and Department of Biomedical Sciences, Creighton University School of Medicine Omaha, Nebraska, USA

Skeletal muscle insulin resistance has been shown to be a primary defect in the majority of patients with type 2 diabetes mellitus (DM2) and overexpression of forkhead box O 1 (FOXO1), a key insulin signaling negative regulator, plays a critical role in skeletal muscle insulin resistance. Recent prospective studies have consistently shown that vitamin D deficiency is closely associated with the incidence of DM2. However, the mechanism underlying vitamin D signaling induced skeletal muscle insulin resistance and the progression of DM2 remains unknown. We generated skeletal muscle-specific VDR-null (SMVDR-/- ) mice that were confirmed by almost complete deletion of VDR in genomic DNA, mRNA, and protein levels in skeletal muscle. We discovered that these mice developed insulin resistance and glucose intolerance accompanied by increased muscle FOXO1 expression, nuclear translocation, and its target gene expression. Importantly, we also found persistent $\mathrm{FOXO1}$ activation in skeletal muscle of global VDR-null mice. Treatment of C2C12 muscle cells with 1,25-dihydroxyvitamin D increased VDR levels and reduced FOXO1 expression and nuclear translocation, indicating that activated VDR signaling negatively regulates FOXO1 activity. The results suggest that persistent FOXO1 activation-induced insulin resistance in skeletal muscle may be responsible for impaired glucose metabolism in SMVDR-/- mice and provide evidence for the utility of vitamin D supplementation for intervention of DM2. 
Combined Poster Session \& Competition

\section{0}

IMPLICATION OF THE SODIUM-HYDROGEN EXCHANGER TYPE 1 NHE1 IN THE DEVELOPMENT OF HYPOTENSION IN HEREDITARY CARDIOMYOPATHY

A-K Johny, J Danielle, B Ghassan

Department of Anatomy and Cell Biology, Faculty of Medicine, Université de Sherbrooke, Sherbrooke, Quebec

Cardiomyopathy in general is defined as a disease of the heart muscle. The purpose of this study is to test the hypothesis that hereditary cardiomyopathy (HCM) is also a vascular disease to which NHE1 may contribute. Our results show a decrease in mean arterial pressure during the development of HCM. The change in blood pressure was accompanied by an increase in NHE-1 density as well as in cytosolic and nuclear sodium levels. The cytosolic and nuclear $\mathrm{Na}+$ overloads were modulated by ET-1 and were surprisingly upregulated only in VSMCs from HCMHs. Treatment with the NHE1 inhibitor EMD87580 partially prevented the decrease in blood pressure and early death. In conclusion, our results suggest that the vascular anomaly taking place during the development of $\mathrm{HCM}$ is at the level of both VECs and VSMCs. In addition, the changes in NHE-1 sensitivity to ET-1 in VSMCs could be compensatory mechanisms to overcome a vascular failure. Supported by a CIHR grant.

\section{1}

ENDOTHELIAL CELL DIFFERENTIATION OF PORCINE ADIPOSE-DERIVED MESENCHYMAL STEM CELLS IS REGULATED BY THE EXPRESSION OF MMP2 AND MMP14

SG Almalki ${ }^{1}$, Y Llamas ${ }^{2}$, DK Agrawal ${ }^{1,2,3}$

${ }^{1}$ Department of Biomedical Sciences; ${ }^{2}$ Department of Medical Microbiology and Immunology; ${ }^{3}$ Department of Clinical and Translational Science, Creighton University School of Medicine, Omaha, Nebraska, USA

RATIONALE: Adipose-derived mesenchymal stem cells (AMSCs) represent promising tools in various clinical applications. The molecular mechanisms that control the ability of AMSCs to remodel extracellular matrix (ECM) barriers during differentiation are not clearly understood. In this study, we investigated the expression of matrix metalloproteinases (MMPs) during the differentiation of AMSCs to endothelial cells (ECs). Methods: AMSCs were characterized by positive staining for MSC markers, CD44, CD90, CD105, and negative staining for CD14, and CD45. The plasticity of MSCs was detected by multi-lineage differentiation. The mRNA transcripts for different MMPs and TIMPs were analyzed by RT-PCR. The enzyme activity and protein expression were also analyzed by gelatin zymography, ELISA, and Western blot.

RESULTS: The differentiation of AMSCs to ECs was confirmed by the mRNA expression of EC markers. The mRNA transcripts of MMP2 and MMP14 were significantly increased during the differentiation. Western blot and ELISA showed an elevated MMP14 and MMP-2 expression. The enzyme activity of MMP2 was also observed. MMP2 and MMP14 silencing showed significant increase in the expression of EC markers, formation of tubes, and acetylated-LDL uptake. Conclusion: We demonstrated that porcine AMSCs have the ability to differentiate into ECs. The data presented herein, for the first time, demonstrated that the up-regulation of MMP2 and MMP14 has an inhibitory effect on the differentiation of AMSCs to ECs, and silencing them increases the differentiation of AMSCs to ECs. These results could provide novel insights aimed at therapeutic strategies for re-endothelialization of coronary arteries or regulation of angiogenesis.

\section{2}

CARNOSINE LOADING IN CARDIAC TISSUE ATTENUATES MYOCARDIAL ISCHEMIA REPERFUSION INJURY

SP Baba, D Zhang, D Hoetker, Y Guo, D Conklin, A Bhatnagar

Department of Medicine, University of Louisville, Louisville,

Kentucky, USA

Myocardial ischemia/reperfusion (I/R) remains the leading cause of death, however there is still no effective therapy to prevent $I / R$ injury. $I / R$ injury increases the generation of reactive aldehydes that are metabolized by enzymes such as aldose reductase, aldehyde dehydrogenase and also sequestered by endogenous dipeptide carnosine. Although the role of these detoxifying enzymes is known however the role of carnosine in $\mathrm{I} / \mathrm{R}$ injury has not been elucidated. Carnosine is a histidyl dipeptide of b-alanine and histidine that is synthesized by the enzyme carnosine synthase (ATPGD1). Carnosine has the abilities to buffer intracellular $\mathrm{pH}$, bind metals and form conjugates with reactive aldehydes. Our previous studies had shown that carnosine protects isolated mice hearts from I/R injury. To completely understand the role of carnosine in cardio-protection we generated cardiospecific ATPGD1 transgenic (TG) mice. Levels of carnosine and anserine were increased 100 fold in the cardiac tissue. Echocardiographic analysis showed that the ATPGD1 overexpression did not affect cardiac function. When subjected to 30 mins of ischemia and $24 \mathrm{~h}$ of reperfusion the infarct size of ATPGD1TG was significantly decreased compared to the WT mice heart. To establish the clinical role of this dipeptide mice were fed with carnosine $(10 \mathrm{gm} / \mathrm{L})$ for 7 day and subjected to $30 \mathrm{mins}$ of ischemia and $24 \mathrm{~h}$ of reperfusion. The infarct size was significantly decreased in carnosine treated compared to the non-treated mice. These finding indicate the cardioprotective role of carnosine and suggest that this dipeptide can be used as a potential therapy for attenuating myocardial $\mathrm{I} / \mathrm{R}$ injury.

\section{3}

TNF- $\alpha$ ENHANCES IGF-1 INDUCED DNMT3A EXPRESSION IN HUMAN CORONARY ARTERY SMOOTH MUSCLE CELLS

\section{CS Boosani, DK Agrawal}

Center for Clinical \& Translational Science and Department of

Biomedical Sciences, Creighton University School of Medicine, Omaha, Nebraska, USA

INTRODUCTION: We previously reported increased expression and activity of TNF- $\alpha$ and IGF-1 with significant reduction in SOCS3 expression in atherosclerotic and restenotic lesions in coronary arteries. Also, we found that TNF- $\alpha$ and IGF- 1 treatment epigenetically restores DNMT1 expression and inhibits SOCS3 in human coronary artery smooth muscle cells (hCASMCs). In this study, we report a novel role of IGF-1 to independently induce the expression of DNMT3a, and when treated in combination with TNF- $\alpha$, it can enhance the expression of DNMT3a.

RESULTS: In hCASMCs, IGF-1 treatment alone induced the expression of DNMT3a. However, when treated with both TNF- $\alpha$ and IGF-1 together, DNMT3a expression was significantly up-regulated with concomitant decrease in SOCS3. The increase in DNMT3a expression was independent of DNMT1 expression, although the expression of both DNMT1 and DNMT3a was induced by the combined treatment of hCASMCs with TNF- $\alpha$ and IGF-1.

CONCLUSION: Since intimal hyperplasia following balloon angioplasty or intravascular stenting is enhanced in the presence of both IGF-1 and TNF- $\alpha$, and increase in SOCS3 would be beneficial in controlling intimal hyperplasia. The present findings suggest a novel mechanism underlying pro-hyperplasia effects of IGF-1, and identifies DNMT3a as a novel target to develop better therapeutic approaches to prevent intimal hyperplasia and restenosis following coronary intervention. 
094

PLASMA OXYLIPINS INCREASE THE ODDS OF HIGH CENTRAL AORTIC BLOOD PRESSURE, CEREBROVASCULAR ACCIDENTS, AND MYOCARDIAL INFARCTIONS AND ARE BENEFICIALLY INFLUENCED BY DIETARY FLAXSEED

$\underline{\text { S Caligiuri }}{ }^{1,2,3}, \mathrm{H}$ Aukema ${ }^{4}$, D Rodriguez-Leyva ${ }^{1,2}$, A Ravandi ${ }^{2,3,5}$, $\overline{\text { R Guzman }}^{6}$, G Pierce, $1,2,3$

${ }^{1}$ CCARM; ${ }^{2}$ ICS, St Boniface Hospital Research Centre;

${ }^{3}$ The Departments of Physiology; ${ }^{4}$ Human Nutritional Sciences;

${ }^{5}$ Internal Medicine; ${ }^{6}$ Surgery, University of Manitoba,

Winnipeg, Manitoba

INTRODUCTION: Uncontrolled hypertension leads to cardiac and cerebrovascular events. A novel therapeutic target may be a class of highly bioactive molecules called oxylipins. Dietary flaxseed may modulate oxylipins by altering the oxylipin substrate profile.

METHODS: The FlaxPAD trial assessed the impact of dietary flaxseed on central blood pressure $(\mathrm{BP})(\mathrm{n}=81)$, the relationship between central BP and plasma oxylipins, and the relationship of oxylipins to cardiac and cerebrovascular events $(n=100)$. Central BP, plasma oxylipins, and events were measured at baseline and 6 months with pulse wave analysis, HPLC-MS/MS, and patient file assessment, respectively.

RESULTS: Central BP was lower in the flaxseed group by (systolic/diastolic) $8 \mathrm{mmHg} / 4 \mathrm{mmHg}$ versus placebo at 6 months $(\mathrm{p}<0.05)$. Significant associations were observed between 17 of the 43 detected oxylipins and central BP. Flaxseed induced a significant decrease in 7 pro-inflammatory/ vasoconstrictive oxylipins. The prevalence of transient ischemic attacks, cerebrovascular accidents, angina, \& myocardial infarctions was $16 \%$, $10 \%, 16 \%, \& 24 \%$, respectively. Eight plasma oxylipins influenced the odds of these events. 16-hydroxyeicosatetraenoic acid increased the odds of high central BP, angina, and cerebrovascular accidents $(\mathrm{p}<0.05)$.

CONCLUSION: Oxylipins may be a novel target or diagnostic/risk marker for central BP, cerebrovascular/cardiovascular events. Flaxseed decreased the concentration of vasoconstrictive plasma oxylipins.

SUPPORTED BY: CIHR, Heart $\mathcal{E}$ Stroke Foundation, Flax 2015, ARDI,

Western Grains Research Foundation $\mathcal{E}$ SBH Foundation

\section{5}

ANGIOTENSIN II RECEPTOR DISTRIBUTION,

INTERNALIZATION AND MODULATION OF INTRACELLULAR CALCIUM: DIFFERENCES BETWEEN HUMAN RIGHT AND LEFT VENTRICULAR ENDOCARDIAL ENDOTHELIAL CELLS

M Chamoun, N Abdel-Karim Abdel-Malak, C Provost, Y Simon, G Bkaily, D Jacques

Department of Anatomy and Cell Biology, Faculty of Medicine, Université de Sherbrooke, Sherbrooke, Quebec

We previously reported that Ang II and AT1R and AT2R are present but differently distributed in right ventricular endocardial endothelial cells (EECRs) and that AT1R mediates the effects of Ang II on intracellular Ca2+ $[\mathrm{Ca} 2+]$ i. In the present study, we determined if similar to EECRs, left ventricular endocardial endothelial cells (EECLs) possess Ang II and its receptors and if they have the same distribution and regulation of $[\mathrm{Ca} 2+]$ i. We also studied the internalization of AT1R and AT2R by Ang II in both cell types. Our results demonstrated that Ang II and its receptors are also present in EECLs with a similar distribution as compared to EECRs. However, their densities are higher in EECLs versus EECRs. Our results also showed that Ang II induced a concentration-dependant increased in [Ca2+]i that is mediated by the AT1R and AT2R. Finally, pretreatment of EECRs and EECLs with Ang II induced de novo synthesis of AT1R in EECRs and degradation in EECLs whereas it induced de novo synthesis of AT2R in both cell types. In conclusion, the present study is the first to show that differences exist between EECRs and EECLs regarding the Ang II system.

FUNDING: Supported by the HSFC.

\section{6}

HOMOCYSTEINE INCREASES MACROPHAGE-DERIVED PARAOXONASE EXPRESSION INDEPENDENT OF CD68

\section{$\underline{\text { I Chernyavskiy }^{1}}{ }^{\text {, L Winchester }}{ }^{1}$, S Veeranki ${ }^{1}$, SC Tyagi ${ }^{1}$}

${ }^{1}$ University of Louisville School of Medicine Department of

Physiology and Biophysics, Louisville, Kentucky, USA

BACKGROUND: Although atherosclerotic plaque rupture is the leading cause of myocardial infarction, the mechanisms are unclear. Macrophages burdened with oxidized LDL (oxLDL) become foam cells and are hallmarks of plaque instability. One of the main macrophage-specific receptors for oxLDL is CD68. Paraoxonase (PON) is a family of high-density lipoprotein (HDL)-associated lactonases capable of retarding/inhibiting LDL oxidation. Homocysteine (Hcy), an amino acid homologue and independent cardiovascular risk factor, is metabolized by PON.

HYPOTHESIS: Given the connections between oxLDL, PON, Hcy, and macrophages to atherosclerosis, we hypothesized that $\mathrm{PON}$ expression is increased by Hcy via CD68.

METHODS: Murine J774a.1 macrophages were treated with LDL, oxLDL, Hcy, or oxLDL+Hcy. Also, separate treatment groups included macrophages that had CD68 silence. Cell lysates were analyzed via Western blotting.

RESULTS: Pon1 is present in macrophages. Hcy along with oxLDL significantly increases PON (51\%, 1.51 vs 0.97 ) expression compared to controls than oxLDL alone. PON expression is significantly decreased ( $33 \%, 0.67$ vs 1 ) with silencing of CD68. PON expression is significantly decreased more with oxLDL ( $82 \% 0.17$ vs 1 ) in presence of CD68 silencing but is significantly increased with oxLDL+Hcy (24\% 1.24 vs 1$)$. CD68 expression tends to increase more with oxLDL+Hcy than oxLDL alone when compared to control and the tendency follows with silencing of CD68. Our results conclude that Hcy increases macrophage-derived PON expression independent of CD68.

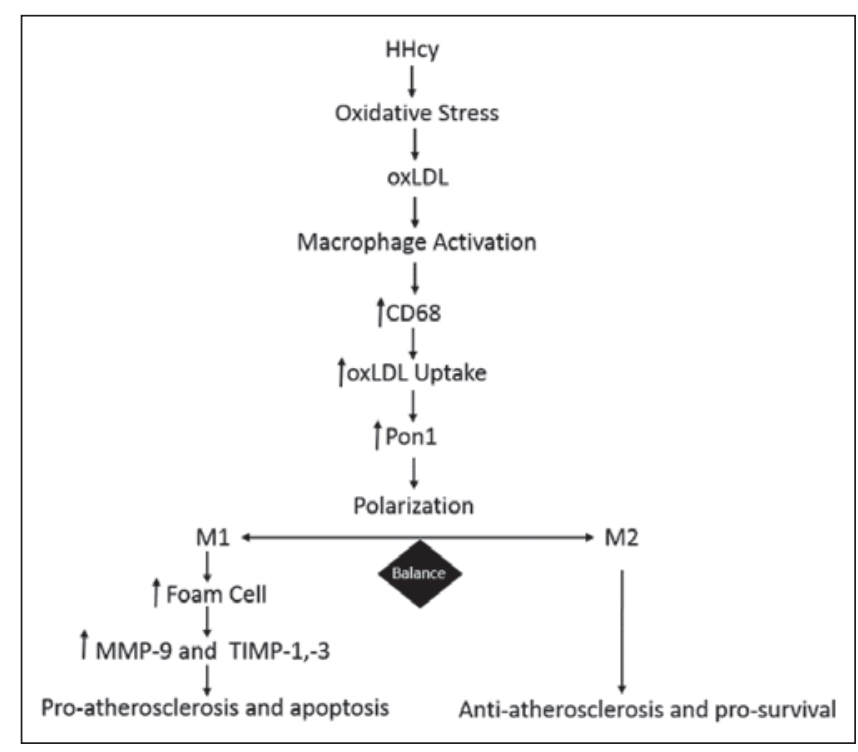

097

\section{EFFECTS OF ACUTE AND CHRONIC HYPERGLYCEMIA ON} LUNG CAPILLARY PERMEABILITY

LClemmer, L Xiang, S Lu, P Mittwede, R Hester

Department of Physiology, University of Mississippi Medical Center, Jackson, Mississippi, USA

Hyperglycemia is correlated with increased vascular oxidative stress and endothelial dysfunction including increased vascular permeability. The role of chronic hyperglycemia in affecting lung permeability is unclear in a type 2 diabetes setting. Lung capillary coefficient (Kf) was measured in the isolated lung of lean Zucker (LZ) and obese Zucker rats (OZ). We hypothesized obesity increases lung permeability through chronic hyperglycemia. OZ had impaired glucose and insulin tolerance which was associated with elevated lung $\mathrm{Kf}(19.4 \pm 3 \mathrm{ml} / \mathrm{mmHg} / \mathrm{g} / \mathrm{min})$ as compared to $\mathrm{LZ}$ 
$(11.4 \pm 2 \mathrm{ml} / \mathrm{mmHg} / \mathrm{g} / \mathrm{min})$. In a separate experiment, OZ were subject to 4 weeks of Metformin treatment ( $300 \mathrm{mg} / \mathrm{kg} /$ day orally) to improve insulin resistance and glycemic control. Metformin treatment significantly improved oral glucose tolerance, insulin sensitivity, and vascular oxidative stress and this was associated with improved baseline lung Kf $(11.5 \pm 1 \mathrm{ml} /$ $\mathrm{mmHg} / \mathrm{g} / \mathrm{min}$ ) as compared to OZ control animals. To examine the role of acute hyperglycemia in lung permeability regulation, lungs from LZ and $\mathrm{OZ}$ were subject to acute hyperglycemia $(550 \mathrm{mg} / \mathrm{dL})$. Thirty minutes of hyperglycemia increased lung Kf in LZ but not in OZ. Acute apocynin treatment $(3 \mathrm{mM})$ added in the isolated lung perfusion solution significantly ameliorated the increased Kf in the acute hyperglycemic-treated LZ. These data suggests that the chronic hyperglycemia in obesity can exacerbate lung $\mathrm{Kf}$ and that acute hyperglycemia can acutely increase lean control lungs through increases in oxidant stress. This warrants further investigation into using antioxidants in the treatment of diabetic and acute hyperglycmemic-induced lung hyperpermeability.

Supported by AHA-14PRE20380069, AHA-12SDG12050525, AHA12POST12060126, NIH P20GM104357, HL-51971, HL-89581 and T32 HL-105324.

\section{8}

\section{EFFECT OF COLCHICINE ON RAT CARDIAC FUNCTION AFTER ACUTE MYOCARDIAL INFARCTION}

EL Couture, B Tricot, MP Ricapito, L Tremblay, A Carpentier, M Nguyen, M Auger-Messier, P Farand

Department of Medicine, Université de Sherbrooke, Sherbrooke, Quebec

INTRODUCTION: Use of non-steroidal anti-inflammatory drugs after acute myocardial infarction (AMI) deteriorates cardiac function. Colchicine inhibits mitosis and neutrophil activity. Colchicine is indicated in post-AMI pericarditis but its safety in regards to cardiac function is unknown.

METHODS: Under general anesthesia, AMI was induced in 27 rats by permanent ligation of the left anterior descending coronary artery. Then, rats were randomly assigned to placebo $(\mathrm{N}=15)$ or colchicine $30 \mu \mathrm{g} / \mathrm{kg}$ daily by intra-peritoneal injection $(\mathrm{N}=17)$. At day 7 , cardiovascular magnetic resonance imaging with 7T scanner was performed in 20 randomly assigned rats. Left ventricular ejection fraction (LVEF), end-diastolic volume (EDV), end-systolic volume (ESV) and late gadolinium enhancement (LGE) were measured. At day 8, all hearts were harvested for pathologic study with TTC staining for evaluation of scar extent.

RESULTS: Death before day 7 occurred in 2 rats in colchicine group and 4 in placebo group. No statistically significant differences between colchicine and placebo were observed: EDV ( 631 vs $574 \mathrm{uL}, \mathrm{p}=0,26$ ), ESV (252 vs 176 $\mathrm{uL}, \mathrm{p}=0,20)$ and LVEF $(60 \%$ vs $69 \%, \mathrm{p}=0,16)$. Scar amount as measured by LGE showed good qualitative correlation with pathologic assessment.

CONCLUSIONS: In this model, colchicine does not deteriorate cardiac function after AMI. A trend toward negative left ventricular remodeling was observed. These results warrant more studies to evaluate colchicine safety after AMI.

\section{9}

\section{ELASTIN-DERIVED PEPTIDES INFLUENCE MACROPHAGE PHENOTYPES IN ABDOMINAL AORTIC ANEURYSM}

M Dale, W Xiong, JS Carson, MK Ruhlman, BT Baxter

Department of Surgery, University of Nebraska Medical Center,

Omaha, Nebraska, USA

OBJECTIVE: Abdominal aortic aneurysm (AAA) is a disease characterized by inflammatory cell infiltration and extracellular matrix (ECM) degradation. Damage to the ECM results in release of elastin-derived peptides (EDPs). EDPs recruit inflammatory cells and promote the differentiation of macrophages to a pro-inflammatory M1 phenotype. BA4, a monoclonal antibody that specifically recognizes six peptide sequences in EDPs, blocks the EDP-mediated effect on macrophages. Our hypothesis is that introducing anti-inflammatory M2 macrophages or blocking the EDP-mediated M1 differentiation of macrophages in AAA will prevent aneurysm formation.

METHODS: Bone marrow-derived macrophages (BMMs) were isolated and treated with various doses of EDPs and their gene expression profiles were analyzed by qPCR. Additionally, BMMs were treated with IFN- $\gamma$ to induce a pro-inflammatory M1 phenotype or IL-4 to induce an antiinflammatory M2 phenotype. M1 or M2 BMMs were administered into mice, which then underwent aneurysm induction via the calcium chloride $(\mathrm{CaCl} 2)$ induced murine model. A third group of mice was given weekly intraperitoneal injections of BA4 $(10 \mathrm{mg} / \mathrm{kg})$. Aortic tissue was then removed and subjected to Western blot, gelatin zymography, and histological analysis six weeks after aneurysm induction.

RESULTS: EDP treatment induced a response similar to IFN $-\gamma$ induced M1 activation via expression of M1 associated markers such as TNF- $\alpha$ and IL-1 $\beta$. Additionally, administration of M1 polarized BMMs to $\mathrm{CaCl}$ treated mice significantly increased aortic dilation compared to administration of M2 polarized BMMs. BA4 treatment reduced aortic dilation similar to the administration of M2 polarized BMMs. BA4 treatment was also able to reduce ECM degradation and macrophage infiltration.

CONCLUSIONS: EDPs cause a pro-inflammatory M1 polarization of BMMs. Reducing the M1 response in AAA by administration of M2 polarized BMMs or inhibition of EDP-mediated signaling may provide potential therapeutic targets for AAA.

\section{0} 6MWT SCORES CAN BE A VIABLE TOOL IN PREDICTING
$\mathrm{VO}_{2}$ MAX SCORES IN PATIENTS WITH HEART FAILURE PK Deka $^{1}{ }^{1}$, BJ Pozehl ${ }^{1}$, D Pathak ${ }^{2}$

${ }^{1}$ University of Nebraska Medical Center, Omaha; ${ }^{2}$ University of Nebraska at Lincoln, Lincoln, Nebraska

BACKGROUND: A cardio-pulmonary exercise (CPX) test is the gold standard in evaluating $\mathrm{VO}_{2}$ max scores. The test is expensive, requires specific equipment that may limit enrollment of subjects and does involve some risk to patients with cardiac problems.

PURPOSE: The purpose of this study was to evaluate the predictive validity of equations provided by Burr et al. (2011) and Ross et al. (2010) in predicting $\mathrm{VO} 2$ max scores from 6 min walk test (6MWT) scores in patients with heart failure (HF).

METHODS: HF patients $(\mathrm{n}=107)$ with NYHA class II-IV performed a VO2 max CPX test and the 6MWT. Correlation between the CPX $\mathrm{VO}_{2}$ max score and the calculated $\mathrm{VO}_{2}$ max scores using the two equations and the 6MWT was analyzed.

RESULTS: Participants were $62.5 \pm 11.5$ years old with a mean of $16.5 \pm 3.5$ $\mathrm{ml} / \mathrm{kg} / \mathrm{min} \mathrm{VO}_{2}$ max score from CPX testing and mean 6MWT distance of $420.7 \pm 90.8$ meters. The Burr et al. (2011) predicted $\mathrm{VO}_{2}$ max was $22.9 \pm 8.7$ and the Ross et al. (2010) predicted $\mathrm{VO}_{2}$ max was $14.6 \pm 2.1 \mathrm{ml} /$ $\mathrm{kg} / \mathrm{min}$. Pearson correlation between the actual $\mathrm{VO}_{2}$ max score from CPX testing and the equation provided by Burr et al. (2011) was .498 while the equation provided by Ross et al. (2010) was .752 .

CONCLUSION: The equation provided by Ross et al. (2010) is simpler and has stronger correlation with the actual $\mathrm{VO}_{2}$ max score from CPX testing. The use of this equation to predict $\mathrm{VO}_{2}$ max score in patients with $\mathrm{HF}$ may be a viable alternative to a $\mathrm{CPX} \mathrm{VO}_{2}$ max exercise test.

ACKNOWLEDGEMENT: Research reported in this study was supported by NHLBI of the National Institutes of Health under award number R01 HL112979. The content is solely the responsibility of the authors and does not necessarily represent the official views of the National Institutes of Health.

\section{1}

\section{CALCITRIOL INHIBITS THE DIFFERENTIATION OF ADIPOSE-DERIVED MESENCHYMAL STEM CELLS INTO ADIPOCYTES}

\section{KP Djossou, P Kokouvi, DK Agrawal}

Center for Clinical \& Translational Science and Department of Biomedical Sciences, Creighton University School of Medicine, Omaha, Nebraska, USA

BACKGROUND: The global obesity pandemic requires action to stem the tide of obesity-related disorders, including cardiovascular diseases and type-2 diabetes. The Third National Health and Nutrition Examination Survey revealed that much of the American population has low vitamin D levels and $33 \%$ are obese. Generation of new adipocytes from multi-potent 
mesenchymal stem cells (MSCs) plays a key role in the development of obesity. Most knowledge of adipocyte differentiation and adipogenesis comes from in vitro studies of fibroblast and pre-adipocytes. There has not yet been a carefully study to evaluate the effect of vitamin D on adipogenesis using adipose-derived MSCs and their role in obesity. Here, we investigated the mechanism by which adipocyte differentiation of MSCs is regulated by in vitro stimulation with calcitriol.

METHODS AND RESULTS: MSCs were isolated from porcine abdominal adipose tissue and characterized by positive staining for MSCs markers, CD44, CD73, CD90; negative staining for CD14, and CD45; and tri-lineage differentiation into adipocytes, chondrocytes, and osteocytes. MSCs from porcine adipose tissue were stimulated with adipose differentiation medium (ADM). No toxicity was observed when MSCs were stimulated with calcitriol at concentrations $0.1-10 \mathrm{nM}$. Cells were then analyzed for adipogenic markers and expression of vitamin D metabolizing enzymes, CYP24A1 and CYP27B1, and VDR by Western Blotting, real-time PCR and flow cytometry. Stimulation of the cells with ADM only significantly increased the expression of adiponectin, leptin, lipoprotein lipase, fatty acid synthetase, PPAR- $\gamma$, and C/EBP $\alpha$. In the presence of calcitriol there was significant decrease in PPARY, C/EBP $\alpha$ and adipogenic markers. The VDR and CYP27B1 expression peaked at 3h and CYP24A1 at 24h after stimulation with calcitriol.

CONCLUSION: MSCs possess the machinery for vitamin metabolism, and stimulation with calcitriol can inhibit the differentiation of adipose MSCs, thus helping to curb obesity.

\section{2}

\section{ROLE OF BRANCHED-CHAIN AMINO ACID OXIDATION IN CARDIAC INSULIN RESISTANCE}

N Fillmore $^{1}$, L Zhang ${ }^{1}$, A Fukushima ${ }^{1}$, CS Wagg ${ }^{1}$, GD Lopaschuk ${ }^{1}$ ${ }^{1}$ Cardiovascular Research Centre, Mazankowski Alberta Heart Institute, University of Alberta, Edmonton, Alberta

In addition to skeletal muscle insulin resistance (IR), obesity is also associated with cardiac IR. Recent studies have proposed that elevated branched-chain amino acids (BCAA) may induce IR due to BCAA oxidation inhibition of fatty acid metabolism. However, in obesity-associated IR, cardiac fatty acid oxidation is actually elevated. In order to examine the role of BCAA oxidation in cardiac IR, mice were fed a high fat diet (HFD) for 10 weeks to induce IR and glycolysis, BCAA oxidation, glucose oxidation, and palmitate oxidation were measured in isolated working hearts. Perfusate contained $5 \mathrm{mM}$ glucose, $0.8 \mathrm{mM}$ palmitate, $0.15 \mathrm{mM}$ leucine, $0.15 \mathrm{mM}$ isoleucine, and $0.2 \mathrm{mM}$ valine. HFD significantly reduced cardiac BCAA oxidation $(30.8 \pm 3.9$ vs $55.3 \pm 7.1 \mathrm{nmol} / \mathrm{min} / \mathrm{g}$ dry wt) decreasing its already low relative contribution to TCA acetyl CoA production from $1.3 \%$ to $0.9 \%$. This decline in BCAA oxidation was accompanied by a rise in circulating BCAA. The expression of branched-chain a-keto acid dehydrogenase (BCKDH) was significantly reduced in HFD hearts, potentially contributing to this decline in BCAA oxidation. Together these results suggest that cardiac IR is not due to increased BCAA oxidation and inhibition of fatty acid oxidation. We hypothesize that reduced BCAA oxidation contributes to IR by leading to increased BCAA levels which stimulate $\mathrm{mTOR}$, decreasing insulin signaling.

\section{3}

\section{ROLE OF HYDROGEN SULFIDE IN THE REGULATION OF DNA METHYL TRANSFERASES IN CARDIOMYOCYTES}

\section{BT Hackfort, P Prathipati, PK Mishra}

University of Nebraska Medical Center, Omaha, Nebraska, USA

DNA methyl transferases (DNMTs) induce cardiac hypertrophy and are upregulated in diabetic hearts. Hydrogen sulfide (H2S) mitigates hypertrophy and ameliorates diabetic heart failure. However, the role of $\mathrm{H} 2 \mathrm{~S}$ in the regulation of DNMTs in diabetic hearts is unclear. We hypothesized that H2S mitigates the high glucose-mediated induction of DNMT-1, DNMT-3a, and DNMT-3b in cardiomyocytes. To test this hypothesis, HL-1 cardiomyocytes were treated with a physiological dose of glucose (LG, $5 \mathrm{mM}$ ), high glucose (HG, $25 \mathrm{mM}$ ), HG+SG1002 (hydrogen sulfide donor, $20 \mu \mathrm{M}$ ), or HG+DMSO (SG1002 control) for 24 hours. Cells were collected for protein and RNA analysis. Our results showed high glucose treatment tended to induce DNMT-1 (1.21 \pm 0.12$)$, DNMT-3a (1.19 $\pm 0.11)$, and DNMT-3b $(1.10 \pm 0.10)$ protein levels as fold change compared to LG $(\mathrm{N}=3)$, however it was not significant. SG1002 had no effect on DNMT-1 $(1.14 \pm 0.09)$ but tended to further increase DNMT-3a $(1.28 \pm 0.04$, $\mathrm{P}=0.03)$ and DNMT-3b $(1.23 \pm 0.16)$ protein levels compared to LG treatment $(\mathrm{N}=3)$. Interestingly, qPCR analyses demonstrated downregulation of DNMT-1 $(0.48 \pm 0.11, \mathrm{P}=0.015, \mathrm{~N}=4)$ and DNMT-3b $(0.27 \pm 0.07$, $\mathrm{P}=0.003, \mathrm{~N}=5)$ mRNA in $\mathrm{HG}$ compared to LG, which was mitigated in $\mathrm{HG}+\mathrm{SG}(0.79 \pm 0.17$ and $0.60 \pm 0.18$, respectively, $\mathrm{N}=4)$. These results elicit a role of $\mathrm{H} 2 \mathrm{~S}$ in regulation of DNMTs in hyperglycemic cardiomyocytes. The increases in protein levels and decreases in the mRNA levels suggest increased protein stability and/or shorter mRNA half-life of DNMTs by H2S treatment. Further analyses with DNMT inhibitor treatment and using a diabetic animal model are required to conclude the specific role of SG1002 on DNMTs in diabetic cardiomyocytes.

\section{4}

\section{PRIMARY CARDIOVASCULAR PREVENTION: A KINESIOLOGIST-BASED INTERVENTION IN THE WORKPLACE}

NC Hamm ${ }^{1,2}$, A Edye-Mazowita ${ }^{2}$, AN Stammers ${ }^{1,2}$, DS Kehler ${ }^{1,2}$, DE Kimber ${ }^{1,2}$, ME Norman ${ }^{1}$, AJ Johnson ${ }^{1}$, AE Ready ${ }^{1}$,

DR Bouchard ${ }^{1}$, SM Strachan ${ }^{1}$, J McGavock ${ }^{1}$, TA Duhamel ${ }^{1,2}$

${ }^{1}$ Health, Leisure \& Human Performance Research Institute, Faculty of Kinesiology \& Recreation Management, University of Manitoba; ${ }^{2}$ Institute of Cardiovascular Sciences, St Boniface Hospital Research Centre, Winnipeg, Manitoba

The majority of Canadians do not participate in enough physical activity to receive health benefits. Physical inactivity is a major contributor to cardiovascular and heart disease risk, whereas greater amounts of physical activity is known to reduce risk. There is a growing recognition that workplaces have the capacity to deliver health promotion initiatives that successfully reduce the prevalence of chronic disease risk factors among employees. This project will determine if the ENCOURAGE physical activity promotion model can be adapted to support workplace wellness programs. A 1-year, quasi-experimental intervention was developed, where participants met with a kinesiologist on at least four separate occasions to learn the skills needed to adopt and maintain a more physically active lifestyle. The primary outcome is a change in physical activity measured with accelerometers.

Baseline data shows participants are only engaging in $43.5 \pm 37.5$ $($ Mean \pm SD) and $30.1 \pm 41.0$ minutes per week of total and moderate to vigorous physical activity in bouts of 10 minutes or more, respectively (Total 10 Min and MVPA 10 Min). Additionally, total and moderate to vigorous physical activity accrued sporadically (Total Sporadic $_{\text {and }}$ MVPA $_{\text {Sporadic }}$ ) was $1282 \pm 406$ and $207 \pm 74$ minutes per week, respectively. No significant differences were seen in Total 10Min, MVPA 10Min, and MVPA $_{\text {Sporadic }}$ for those who have completed their 2-month time point $(n=8)$; however, there was a significant increase in Total ${ }_{\text {Sporadic }}$ physical activity levels to $1574 \pm 509$ minutes per week.

Results suggest a kinesiology based physical activity program in the workplace can increase Total ${ }_{\text {Sporadic }}$ physical activity levels amoung employees, thus decreasing cardiovascular disease risk. Data collection is scheduled until July, 2016.

ACKNOWLEDGEMENTS: This project is funded by a Heart and Stroke Foundation Primary Prevention Challenge grant.

\section{5}

FEASIBILITY OF ARTIFICIAL CARBON DIOXIDE BALNEOTHERAPY IN HEMODIALYSIS PATIENTS WITH HEART FAILURE

H Hayashi ${ }^{1}$, Y Moriyama ${ }^{2}$, T Yamada ${ }^{2}$, K Kasuga $^{2}$, D Kamoi ${ }^{2}$, H Kawahara ${ }^{2}$

${ }^{1}$ Seijoh University; ${ }^{2}$ Nagoya Kyouritsu Hospital, Nagoya, Japan

BACKGROUND: Artificial carbon dioxide balneotherapy (CDB) has been shown to have positive effects on the cardiovascular system via peripheral 
vasodilatation, but its efficacy in the treatment of hemodialysis (HD) patients with heart failure and preserved ejection fraction (HFpEF) has not been clarified. Therefore, we assessed the feasibility of CDB in HD patients with HFpEF.

METHOD: Two hundred sixteen patients were screened to exclude those with an E/e' of $<8$ and NYHA class III/IV. Seven eligible patients (mean age: $71 \pm 11$ years) received CDB daily for 2 weeks. CDB efficacy was evaluated based on the peak oxygen uptake/heart rate ratio (VO2/HR), minute ventilation versus carbon dioxide production (VE vs. VCO2) slope, brain natriuretic peptide (BNP) level, high-sensitivity cardiac troponin I (hscTnI) level, and ejection fraction.

RESULT: In 4 patients with an E/e' of $>13$, the hs-cTnI level $(0.62 \pm 0.02$ to $0.48 \pm 0.02 \mathrm{ng} / \mathrm{ml}, \mathrm{p}=0.03)$ and $\mathrm{VE}$ vs. $\mathrm{VCO}_{2}$ slope $(34.80 \pm 1.87$ to $30.93 \pm 2.09, \mathrm{p}=0.05)$ decreased and the $\mathrm{VO}_{2} / \mathrm{HR}$ improved $(5.43 \pm 0.69$ to $5.98 \pm 0.77, \mathrm{p}=0.04)$ significantly after 4 weeks of CDB. Further, the BNP level decreased (416.5 to $251.5 \mathrm{pg} / \mathrm{ml}$ ), although this change was not statistically significant. The 3 patients with an E/e' $\leq 13$ showed no significant change in the evaluated parameters.

CONCLUSION: CDB could be feasible in HD patients with HFpEF, with an $E / e^{\prime}$ of $>13$.

\section{6}

\section{PTEN INHIBITOR REDUCES CARDIAC REMODELING IN DOXORUBICIN-INDUCED CARDIOMYOPATHY}

\section{TA Johnson, DK Singla}

Burnett School of Biomedical Sciences, College of Medicine,

University of Central Florida, Orlando, Florida, USA

BACKGROUND: Doxorubicin (Doxo) is one of multiple anthracycline drugs used to effectively treat various forms of cancer. Unfortunately, Doxo treatment stimulates adverse cardiac remodeling and subsequent heart failure. We have previously demonstrated that transplanted embryonic stem (ES) cells and their conditioned medium (CM) modulate the PTEN pathway and reduce apoptosis, fibrosis and hypertrophy in a Doxo-Induced Cardiomyopathy (DIC) model. VOOHpic (VO), the most potent known PTEN inhibitor, has shown to be cardioprotective against ischemia-reperfusion injury; however VO efficacy in a DIC model has not been explored. HYPOTHESIS: Intraperitoneal (IP) delivery of VO blunts PTEN expression and protects the heart from doxorubicin-induced cardiac remodeling. METHODS: Animals were divided into three groups; Group 1: Control (Saline), Group 2: Doxo (12 mg/kg, Cumulative dose) and Group 3: Doxo and VO (12 mg/kg and $10 \mu \mathrm{g} / \mathrm{kg}$ cumulative doses $)$ via IP injection. One week post-DIC, mice were subjected to echocardiography to examine cardiac function, sacrificed and hearts were harvested for further analysis. RESULTS: Immunohistochemistry staining revealed a significant $(\mathrm{p}<0.05)$ decrease in apoptotic cardiomyocytes in Doxo-VO treated hearts compared with Doxo. Furthermore, Hematoxylin and Eosin (H\&E) and Masson's Trichrome histological stains confirmed reduced hypertrophy, interstitial and vascular fibrosis in Doxo-VO treated subjects compared to Doxo group. Western Blotting confirmed the reduction in PTEN levels in Doxo-VO subjects compared to Doxo hearts. Heart function was significantly improved upon Doxo-VO treatment compared to Doxo group. CONCLUSION: Our data suggest that $\mathrm{VO}$ treatment attenuates adverse cardiac remodeling and improves heart function in the DIC heart.

\section{7}

\section{DOWNREGULATION OF THE UNFOLDED PROTEIN RESPONSE IN TAURINE DEFICIENT HEARTS}

IC Ju ${ }^{1}$, I Takashi ${ }^{2}$, S Stephen ${ }^{1}$

${ }^{1}$ University of South Alabama, Department of Pharmacology, College of Medicine, Mobile, Alabama; ${ }^{2}$ Hyogo University of Health Sciences, School of Pharmacy, Kobe, Japan

The unfolded protein response is a cellular quality control process that regulates the proper function of proteins in the endoplasmic reticulum. Accumulation of unfolded and misfolded proteins in the endoplasmic reticulum leads to the activation of a process known as the unfolded protein response. An ER chaperone, GRP78, regulates the unfolded protein response by interacting with three transmembrane sensor proteins (PERK,
IRE-1, ATF6), which upon initiation activate downstream pro-survival or pro-death pathways. In pathologies, such as aging and degenerative diseases, downregulation of the unfolded protein response is associated with oxidative modification of GRP78. However, the unfolded protein response in the taurine deficient heart, which is associated with development of a cardiomyopathy, remains to be clarified. Taurine is a sulfur-containing amino acid and has cytoprotective actions, including antioxidant activity. Indeed, taurine-deficient hearts are oxidatively stressed as shown by decreased aconitase activity, decreased glutathione redox ratio and increased levels of carbonylated proteins. Enhanced oxidative stress in taurine deficient hearts is associated with the downregulation of the unfolded protein response, as evidenced by reduced levels of GRP78 and suppressed activation of the PERK- and IRE-1-mediated pathways. However, these reductions were abolished when oxidative stress in taurine deficient hearts was prevented by mitoTEMPO, a mitochondrial-specific antioxidant. In conclusion, the present study demonstrates that taurine depletion induces oxidative stress, which interferes with protein folding and the unfolded protein response, events that likely contribute to the development of a cardiomyopathy.

Acknowledgements: This work was supported in part by American Heart Association funding.

\section{8 \\ PHOSPHORYLATION OF IRS-1 BY NOVEL PROTEIN KINASES INDUCES NAFLD THROUGH IR IN SWINE DEPENDENTLY OF THEIR VITAMIN D STATUS \\ T Kouassi, DK Agrawal}

Center for Clinical \& Translational Science, Creighton University School of Medicine, Omaha, Nebraska, USA

Non-alcoholic fatty liver disease (NAFLD) results in a spectrum of liver pathology ranging from benign steatosis to non-alcoholic steatohepatitis (NASH), and carries a significant potential to progress to liver cirrhosis and hepatocellular carcinoma. Diacylglycerol (DAG) is a potent activator of novel protein kinases (nPKCs) that inhibit insulin receptor substrate-1 (IRS-1) to induce insulin resistance (IR), and IR dominates the pathogenesis of NAFLD. Here, we investigated the effect of high cholesterol high fructose (HCHF) diet with/without vitamin D deficiency on liver pathology and examined the role of nPKCs in the development of NAFLD. Yucatan microswine were fed HCHF diet either deficient or supplemented with vitamin $\mathrm{D}$-deficient and vitamin $\mathrm{D}$-supplemented diet. The liver histology was examined and correlated with the expression of nPKCs and effects on IRS-1 signaling.

RESULTS: There was significant accumulation of fat in the liver of vitamin D-deficient and HCHF-fed compared to vitamin D-sufficient and supplemented swine. Steatosis was much more in the form of microvesicular lipid droplet with nucleus in the center. HCHF swine develop more fibrosis (METAVIR score=3) than vitamin D-deficient (METAVIR score=2), while vitamin D-sufficiency and -supplementation protected the liver from fibrosis (METAVIR score=0). There was consistently high expression of $\mathrm{PKC}-\delta$ in the liver of HCHF and vitamin D-deficient swine with a strong co-localization with phosphorylated IRS-1. Significant variability was observed in the expression of PKC- $\varepsilon$ and PKC- $\theta$ in the liver.

CONCLUSION: These data suggest that swine fed HCHF and vitamin D-deficient diet are more susceptible to develop NAFLD, which can progress to NASH while vitamin D has a protective role. Insulin resistance through IRS-1 phosphorylation by PKC- $\delta$ might play a key role in inducing NAFLD.

\section{9}

\section{RESUSCITATION OF A DEAD CARDIOMYOCYTE "MITOCHONDRIAL INTERVENTION AND CALPAINS" GH Kunkel, P Chaturvedi, SC Tyagi}

Department of Physiology and Biophysics, University of Louisville, Kentucky, USA

Regulation of mitochondrial dynamics is essential for cardiovascular disease (CVD) related issues like heart failure (HF). Up regulation of proteolytic activity such as calpains and matrix metallo-proteinases (MMP's) are found within the HF model. Although calpains have been known to 
underline the degradative mechanisms leading to cell death, the molecular mechanisms are unclear. Additionally, the mitochondrial roles behind myocyte death need much to be determined. We propose that activation of calpains leads to PCP4 degradation, speculating that PCP4 is a modulator of calmodulin kinase II and calmodulin. Mitochondrial Transcription factor A (TFAM) plays a major role in proteolytic activation contributing to myocyte death. Our preliminary data of live versus dead cardiomyocytes suggests involvement of calpain-1, MMP9, mitochondrial transcription factor A (TFAM) and lon protease. We found upregulated expression of calpain 1 in dead myocytes along with decreased PCP4. The upregulated levels of calmodulin kinase and subsequently calmodulin suggested that these events harbor more calcium into the myocyte and lead to myocyte death. The down regulated levels of TFAM in dead myocytes suggest increased ROS production. In view of the above observations we project that regulating the levels of calpains and TFAM can lead to resuscitation of a dead myocyte. We are further determining whether exosomal delivery of TFAM can mitigate mitochondrial proteolytic activation and revive a dead myocyte.

\section{0}

\section{CHRONIC NICOTINE EXPOSURE EXACERBATES TRANSIENT FOCAL CEREBRAL ISCHEMIA-INDUCED BRAIN INJURY}

\section{Li, H Sun, WG Mayhan}

Center for Cardiovascular Diseases and Sciences, Louisiana State University Health-Shreveport

Tobacco smoking is a risk factor contributing to the development and progression of ischemic stroke. Among many chemicals in tobacco, nicotine may be the key contributor. We hypothesized that nicotine alters the balance between oxidant and antioxidant networks leading to an increase in brain injury following transient focal cerebral ischemia. Male SpragueDawley were treated with nicotine for 4 weeks via implanted subcutaneous osmotic minipump and subjected to a 2-hour middle cerebral artery occlusion $(\mathrm{MCAO})$. Infarct size and neurological deficits were evaluated at 24 hours of reperfusion. Expression of oxidant and antioxidant proteins was measured using Western Blot analysis. Superoxide production was determined by lucigenin-enhanced chemiluminescence. We found that chronic nicotine exposure significantly increased infarct size and worsened neurological deficits. Interestingly, infarct size and neurological deficits were not further increased in $4 \mathrm{mg} / \mathrm{kg} /$ day nicotine group compared to $2 \mathrm{mg} / \mathrm{kg} / \mathrm{day}$ nicotine group. In addition, chronic nicotine exposure didn't alter protein expression of SOD-1 and NADPH oxidase, but significantly down-regulated SOD-2 in cerebral cortex and arteries. Furthermore, nicotine significantly elevated superoxide production of cerebral cortex under basal conditions. Cerebral ischemia/reperfusion produced an increase in superoxide production of cerebral cortex in control group. However, no further increase in superoxide production of cerebral cortex was found in nicotine group. Our findings suggested that nicotine induced exacerbation in brain damage following transient focal cerebral ischemia may be related to an increase in oxidative stress via down-regulation of SOD-2.

\section{1}

WNT/ß-CATENIN PATHWAY PROMOTES THE DIFFERENTIATION OF ADIPOSE-DERIVED MESENCHYMAL STEM CELLS STIMULATED WITH

\section{1,25-DIHYDROXYVITAMIN D AND VEGF-CELLS TOWARD} THE ENDOTHELIAL PHENOTYPE

Y Llamas ${ }^{1}$, S Almalki ${ }^{2}$, DK Agrawal ${ }^{1,2}$

${ }^{1}$ Center for Clinical and Translational Science, ${ }^{2}$ Department of Biomedical Sciences, Creighton University School of Medicine, Omaha, Nebraska, USA

BACKGROUND: Cell-based therapy using adipose-derived mesenchymal stem cells (AMSCs) is an attractive option for re-endothelialization postangioplasty procedures. Wnt $/ \beta$-catenin pathway in AMSC may regulate AMSC-based re-endothelialization of injured arteries. The role of $\mathrm{Wnt} / \beta$ catenin pathway in the differentiation of adipose-derived AMSCs into endothelial cells (ECs) is unknown. In this study, we investigated the effect of Wnt/ $\beta$-catenin pathway on AMSC treated with vitamin D and VEGF in the differentiation of MSCs into ECs.
METHODS AND RESULTS: MSCs were CD11b-CD34-CD44+CD73+ CD90+ and showed characteristics of MSCs. MSCs were stimulated and differentiated into ECs with endothelial growth media $(E G M+50 \mathrm{ng} / \mathrm{ml}$ of VEGF) and EGM media containing $10 \mathrm{nM}$ of calcitriol (EGM $+50 \mathrm{ng} / \mathrm{ml}$ of VEGF +10nM calcitriol) for 10 days. Calcitriol enhanced EGM+VEGFinduced differentiation of MSCs into ECs, as revealed by 3-fold increase in mRNA and 4-fold increase in protein expression of EC markers. Angiogenesis assay and acetylated low density lipoprotein (LDL) uptake assay were used to assess endothelial functionality that showed significant increase in capillary tube sprouting, and increased LDL uptake by differentiated cells in response to EGM +VEGF+calcitriol. Findings from Wnt Pathway array revealed a 3 -fold decrease in $\beta$-catenin and 4 -fold increase in KREMEN1 protein in the cells treated with EGM + VEGF+calcitriol. $\beta$-catenin silencing showed significant increase in the expression of EC markers, formation of capillary tubes, and LDL uptake.

CONCLUSION: The downregulation of $\beta$-catenin and upregulation of KREMEN1 significantly enhanced the differentiation of MSCs into endothelial cells. These results provide novel insight into therapeutic strategies for patients undergoing coronary intervention to limit thrombosis and intimal hyperplasia.

\section{2}

\section{ALPHA LINOLENIC ACID DECREASES THE PRODUCTION OF PRO-APOPTOTIC OXIDIZED PHOSPHOLIPIDS IN CARDIOMYOCYTES AFTER ISCHEMIA/REPERFUSION} R Ganguly1,2,3, D Hasanally ${ }^{1,3}$, TG Maddaford ${ }^{1,2,3}$, R Chaudhary ${ }^{1}$, GN Pierce ${ }^{1,2,3}$, A Ravandi $i^{1,3,4}$

${ }^{1}$ Institute of Cardiovascular Sciences; ${ }^{2}$ The Canadian Center for Agri-food Research in Health and Medicine (CCARM); ${ }^{3}$ St Boniface Hospital Research Centre and the Departments of Physiology and Pathophysiology and ${ }^{4}$ Internal Medicine, Faculty of Health Sciences, University of Manitoba, Winnipeg, Manitoba

Alpha linolenic acid (ALA) is a plant derived omega-3 fatty acid, found in high levels within flaxseed, and when ingested is thought to elicit cardiovascular benefits. Although ALA is suggested to be cardioprotective during an ischemic insult, the mechanism(s) by which this protection occurs is unknown. In this study, primary cultured rat cardiomyocytes were exposed to media with or without ALA for 24 hours and then were subjected to 60 minutes of non-ischemic control conditions (CTR), simulated ischemia (ISCH) for 60 minutes or 60 minutes of simulated ischemia and reperfusion conditions (IR). Pre-treating the cells with ALA resulted in significant incorporation of ALA within cardiomyocyte phosphatidylcholine (PC). There was an increase in cell death after ISCH and IR. The-pro-apoptotic oxidized phospholipids (OxPC), 1-palmitoyl-2-(5'-oxo-valeroyl) snglycero-3 phosphocholine (POVPC) and 1-palmitoyl-2-glutaryl-sn-glycero3-phosphocholine (PGPC) were significantly increased in ISCH and IR. Pre-treating cardiomyocytes with ALA significantly reduced cell death after ISCH or IR, however, caspase-3 cleavage was not different. ALA pretreatment significantly decreased the rise of resting Ca2+ during ischemia and reperfusion, however there was little change to the transient $\mathrm{Ca} 2+$. Pre-treatment with ALA significantly decreased the production of PGPC and POVPC after ISCH or IR. These results suggest that ALA elicits protection to cardiomyocytes by inhibiting the production of pro-apoptotic OxPC species.

This research was supported by grants from CIHR, ARDI, Western Grains Research Foundation and the Heart and Stroke Foundation of Canada. 
113

\section{MIRNA-33A AS A NOVEL THERAPEUTIC TARGET IN DILATED CARDIOMYOPATHY}

A Mittal ${ }^{1}$, S Rana ${ }^{4}$, R Sharma ${ }^{1}$, V Arige ${ }^{5}$, S Khanna ${ }^{2}$, N Mahapatra ${ }^{5}$, S Sarkar $^{4}$, A Bahl ${ }^{1}$, SK Goswami' ${ }^{6}$, M Khullar ${ }^{2}$

${ }^{1}$ Department of Cardiology; ${ }^{2}$ Department of Experimental Medicine and Biotechnology; ${ }^{3}$ Department of Histopathology, Post Graduate Institute of Medical Education and Research, Chandigarh;

${ }^{4}$ Department of Zoology, University of Calcutta; ${ }^{5}$ Department of Biotechnology, Indian Institute of Technology, Madras; ${ }^{6}$ School of Life Sciences, Jawahar Lal Nehru University, New Delhi, India OBJECTIVE: Dilated cardiomyopathy (DCM) accounts for approximately $1 / 3$ rd of total cases of heart failure (HF) and is a leading indication for cardiac transplantation. Myocardin (MYOCD), a potent transcriptional coactivator of smooth muscle (SM) and cardiac genes, is upregulated in failing myocardium in animal models and human end-stage heart failure (HF). microRNAs (miRNAs) are 20-22 nucleotide long non-coding RNAs found to regulate gene expression. However, the role of miRNAs regulating MYOCD expression in heart failure remains unknown. The goal of this study was to identify the miRNAs regulating the cardiac specific MYOCD and to study the molecular and functional consequences of cardiac specific modulation of MYOCD specific miRNA in an animal model of DCM.

APPROACH AND RESULTS: Our study design included identification and validation of miRNA targeting MYOCD using bioinformatics tools and to study its cardiac expression in idiopathic DCM (IDCM) endomyocardial biopsies, renal artery ligation rat model of DCM (RAL). We identified and validated miRNA-33a as a putative regulator of MYOCD expression in cardiomyocytes. Cardiac miRNA-33a expression was significantly decreased in IDCM and in RAL. We investigated if cardiac specific augmentation of miRNA-33a expression using a homing peptide conjugated siRNA could potentially modulate the cardiac remodelling and outcome in RAL. We observed that targeted modulation of miRNA-33a attenuated cardiac hypertrophy and fibrosis, decreased expression of hypertrophy and fibrotic genes and ameliorated the impaired diastolic dysfunction in RAL model of cardiomyopathy.

CONCLUSION/SIGNIFICANCE: This data provide the first evidence that miRNA-33a is involved in regulating cardiac MYOCD expression and cardiac specific augmentation of miRNA-33a offers a putative therapeutic target in DCM.

\section{4}

LOWER DIABETES PREVALENCE ABOVE 3,000 M OF ALTITUDE IN PERU CAN BE EXPLAINED BY WEALTH INDEX, AGE AND SEX DISTRIBUTION: ANALYSIS OF THE PERUVIAN DEMOGRAPHIC AND HEALTH SURVEY LM Mori-Llontop ${ }^{1}$, SN Seclén-Santisteban ${ }^{1}$, ME Rosas ${ }^{1}$, AJ Arias ${ }^{2}$ ${ }^{1}$ Universidad Peruana Cayetano Heredia (UPCH), Lima, Peru; ${ }^{2}$ Instituto Nacional de Estadística e Informática (INEI), Lima, Peru We used the Peruvian Demographic and Health Survey (DHS) 2014 database to ascertain if self-reported diabetes prevalence was different above and below $3,000 \mathrm{~m}$ of altitude, and tried to explain any difference by controlling for confounder variables age, sex, education years, latitude, wealth index, hypertension, tobacco and alcohol use, overweight and obesity status.

The final database included 29,806 people from rural and urban settings across the country. Both populations had rather similar age by sex distribution; however, highland population was markedly less wealthy, had lower education level, less obesity, arterial hypertension, alcohol and smoking rates, and lived predominantly at rural settings, compared with the lowland counterpart.

Diabetes prevalence (percent $[95 \% \mathrm{CI}]$ ) above and below 3,000 $\mathrm{m}$ of altitude was $2.4 \%(1.8-3.3)$ and $4.6 \%(4.1-5.2)$, respectively $(\mathrm{OR}=0.51 \%$ [0.36-0.72]). After adjusting for the before mentioned characteristics, however, the $\mathrm{OR}$ became non-significant $(\mathrm{OR}=0.91$ [0.64-1.3]). Diabetes diagnoses were strongly associated with wealth index, followed by female status, hypertension diagnoses and older age.

We conclude that the observed difference, in our country, can be explained in part by poverty rates associated with lower nutritional resources. The role of the low availability of health services in such deprived and underserved areas, which would explain the lower diabetes diagnoses rate, is discussed.

\section{5}

TRANSCRIPTIONAL REGULATION OF MMP-2 BY SCLERAXIS RS Nagalingam, RA Bagchi, PL Roche, HA Safi, MP Czubryt Institute of Cardiovascular Sciences, St Boniface Hospital Research Centre and Department of Physiology and Pathophysiology, University of Manitoba, Winnipeg, Manitoba

Extracellular matrix (ECM) homeostasis is altered during cardiac fibrosis. ECM synthesis is increased, as is the expression of matrix remodeling enzymes such as Matrix Metalloproteinases (MMPs). Scleraxis is a transcriptional regulator found in cardiac fibroblasts and myofibroblasts that we previously demonstrated plays a vital role in the production of ECM proteins including collagen $1 \alpha 2$ by binding to E-box consensus sequences located in the target gene promoter. MMP-2 (Type IV collagenase-Gelatinase-A) maintains the homeostasis of extracellular matrix by controlling the degradation of matrix protein. Our present proof-of-concept study reveals that the MMP-2 gene is transactivated by scleraxis. Overexpression of scleraxis or 48 hours in NIH-3T3 cells induced MMP-2 expression at the mRNA and protein levels. Using in silico analysis, we identified putative E-box sites in the MMP-2 gene promoter to which scleraxis may bind, and found that scleraxis was capable of directly transactivating this promoter using luciferase reporter assay. Our data suggests that scleraxis may govern the expression of both ECM components such as collagen, as well as ECM remodeling enzymes such as MMP-2. This finding is consistent with a potential role for scleraxis in cardiac fibrosis where MMP-2 and fibrillar collagen gene expression is elevated concurrently with scleraxis.

Supported by the Canadian Institutes of Health Research (grant MOP-136862).

\section{6}

\section{INHIBITION AND ACTIVATION OF THE CARDIAC $\mathrm{NA}^{+} / \mathrm{CA}^{2+}$ EXCHANGER - TWO ASPECTS OF TRANSPORT MODULATION}

N Nagy ${ }^{1}$, Z Kohajda ${ }^{1}$, K Acsai ${ }^{1}$, A Kormos ${ }^{2}$, A Tóth ${ }^{2}$, K Oravecz ${ }^{2}$, P Pollesello $^{3}$, J Levijoki ${ }^{3}$, L Virág ${ }^{2}$, N Jost ${ }^{1,2}$, J Gy Papp ${ }^{1,2}$, A Varró ${ }^{1,2}$ 1MTA-SZTE Research Group of Cardiovascular Pharmacology, Hungarian Academy of Sciences, Szeged, Hungary; 2Department of Pharmacology and Pharmacotherapy, University of Szeged, Szeged, Hungary; 3Orion Pharma, Espoo, Finland

The important role of NCX in several types of arrhythmias is well established. Therefore, its inhibition is proposed as a promising novel antiarrhythmic strategy. Furthermore, a possible positive inotropic effect can be also expected. Also activation of NCX could be advantageous by supporting $\mathrm{Ca} 2+$-extrusion during $\mathrm{Ca} 2+$-overload. However, the exact evaluation of the putative antiarrhythmic role and inotropic action of NCX modulation was hampered by the lack of selective inhibitors. Our aim was to characterize the novel selective NCX-inhibitors, ORM10103, ORM10962, and a putative NCX-activator ORM10792.

Under close to physiological conditions, NCX-inhibition had little influence on CaT and CS. However, favoring reverse NCX either by increasing $[\mathrm{Na}+]$ i with ATX-II, or lowering $[\mathrm{Na}+]_{0}$, the amplitude of $\mathrm{CaT}$ and CS was decreased. Stimulating the forward mode by enhancing $\mathrm{Ca} 2+\mathrm{i}$ via forskolin application, NCX-inhibition resulted in positive inotropy through enhancing CaT magnitude. NCX-inhibition decreased the Ca2+-overload-induced spontaneous diastolic Ca2+-releases. The NCX-activation enhanced either mode of NCX without significantly interfering with L-type Ca2+ current. We found an antiarrhythmic effect of selective NCX-inhibition under condition when both reverse and forward modes of NCX were favored. In contrast, positive inotropic effect could be observed during conditions when only forward mode was facilitated. The selective NCX-modulator compounds may provide novel possibilities to investigate the physiological and therapeutic role of NCX-modulation.

Acknowledgement: This work was supported by the Postdoctoral Program of the Hungarian Academy of Sciences (for N.N). 


\section{7}

CENTRAL ANGIOTENSIN-II RAISES BLOOD PRESSURE AND SYMPATHETIC OUTFLOW VIA THE RHOA/RHO KINASE PATHWAY IN CONSCIOUS RABBITS

PR Pellegrino, AM Schiller, KK Haack, IH Zucker

Cellular \& Integrative Physiology, University of Nebraska Medical

Center, Omaha, Nebraska, USA

INTRODUCTION: Elevated sympathetic tone and activation of the renin-angiotensin system are pathophysiologic hallmarks of diseases like chronic heart failure, hypertension, chronic kidney disease, and obstructive sleep apnea, all of which have compelling burdens and therapeutic needs. The RhoA/Rho kinase pathway is an important mediator of the effects of Angiotensin-II (AngII) in the periphery, but the functional role of this pathway in the brain in AngII-induced sympatho-excitation is not well-characterized.

HYPOTHESIS: We hypothesized that central inhibition of the RhoA/Rho kinase pathway prevents AngII-mediated autonomic dysfunction in rabbits. METHODS: Each rabbit received all four of the following intracerebroventricular infusion treatments for two weeks in random order: AngII, the Rho kinase inhibitor Fasudil (Fas), AngII and Fas, and vehicle infusion via osmotic minipump. After two weeks, the treatment was washed out with vehicle infusion for 7-14 days. Baseline recordings of mean arterial pressure (MAP) and heart rate (HR) were acquired throughout treatment. After ten days of infusion, cardiac sympathetic tone and sympathetic vasomotor tone were assessed by the change in HR after metoprolol and change in MAP after hexamethonium, respectively.

RESULTS: AngII treatment resulted in a pressor effect that was blocked with Fas co-infusion. Both cardiac sympathetic tone and sympathetic vasomotor tone were increased with AngII infusion; this sympatho-excitation was abrogated by co-infusion of Fas. Each of these measures showed a significant interaction between AngII and Fas, indicating that the pressor and sympatho-excitatory responses of central AngII are mediated by the RhoA/ Rho kinase pathway.

CONCLUSIONS: These data indicate that inhibition of the Rho kinase pathway centrally could act as a therapeutic brake on the positive feedback between central renin-angiotensin system activation and sympathetic outflow in many diseases.

\section{8}

\section{ABLATION OF MMP9 ALLEVIATES MITOPHAGY AND MITIGATES CARDIAC DYSFUNCTION IN DIABETICS P Prathipati ${ }^{1}$, BT Hackfort ${ }^{1}$, SS Nandi ${ }^{1}$, HR Shahshahan ${ }^{1}$, PK Mishra ${ }^{1}, 2$}

${ }^{1}$ Department of Cellular and Integrative Physiology; ${ }^{2}$ Department of Anesthesiology, University of Nebraska Medical Center, Omaha, Nebraska, USA

Mitochondrial abnormalities and induced matrix metalloproteinase-9 (MMP9) contribute to diabetic heart failure. However, the specific role of MMP9 in mitochondrial autophagy (mitophagy) is unclear. We hypothesized that deletion of MMP9 gene alleviates excessive mitophagy and mitigates cardiac dysfunction in diabetics. To test this hypothesis, we created Ins $2+/-1$ MMP9-/- mice by ablating MMP9 gene from Ins2+/- Akita (type 1 diabetic) mice. To investigate the specific role of MMP9 in diabetes-induced mitophagy, we used C57BL/6J (WT), Ins2+/- Akita, Ins2+/-/MMP9-/- and MMP9I- mice, and measured the level of mitophagy markers: PINK1, Parkin and LC3B in the heart by qPCR, Western blotting and immunohistochemistry. To determine the role of MMP9 on cardiac dysfunction in diabetics, we performed $\mathrm{M}$-mode echocardiography and measured percentage ejection fraction $(\% \mathrm{EF})$ and fractional shortening (\%FS) in the above four groups. Our results showed elevated mRNA levels of PINK1 $(1.25 \pm 0.42)$, Parkin $(1.95 \pm 0.48)$ and LC3B $(1.89 \pm 0.54)$ in Akita as fold change compared to the WT heart suggesting upregulated mitophagy in Akita. However, their levels were downregulated in Ins2+/-/MMP9-/- (PINK1: 0.06 \pm 0.02 , Parkin: 0.76 \pm 0.18 and LC3B: $0.27 \pm 0.02$ ), which was comparable to MMP9-/- hearts demonstrating downregulation of mitophagy. The protein levels of PINK1, Parkin and LC3B were similar in pattern as mRNA levels. Echocardiography results showed cardiac dysfunction in Akita (\%EF: $56.9 \pm 4.77$, \%FS: $29.3 \pm 3.21$ ), and its

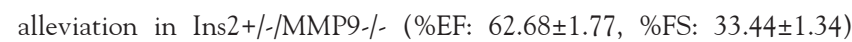
without significant difference in cardiac function between WT and MMP9-/-. In conclusion, abrogation of MMP9 downregulates mitophagy and ameliorates cardiac dysfunction in diabetic hearts.

Acknowledgements: NIH grants: HL113281 and HL116205

\section{9}

\section{REMODELING OF PULMONARY VASCULAR ENDOTHELIUM IN LEFT HEART FAILURE}

$\underline{\text { SS Binil Raj }}{ }^{1}$, GS Ajithkumar ${ }^{1}$, G Sanjay ${ }^{2}$, CC Kartha ${ }^{1}$

${ }^{1}$ Cardiovascular Disease Biology Division, Rajiv Gandhi Centre for Biotechnology; ${ }^{2}$ Department of Cardiology, SCTIMST,

Thiruvananthapuram, India

Pulmonary hypertension associated with left heart disease (PH-LHD) represents the most common form of $\mathrm{PH}$ and is characterized by lung endothelial dysfunction and vascular remodeling. LHD results in passive backward transmission of elevated left atrial pressure and partial obstruction to pulmonary venous drainage. This hemodynamic disturbance in circulation causes increased shear stress and turbulent (disturbed) flow in pulmonary circulation. We hypothesize that pulmonary vascular endothelial cells (PVECs) are exposed to disturbed flow in LHD leading to PVEC dysfunction, pulmonary vascular remodeling and $\mathrm{PH}$. We studied the expression pattern of different shear sensitive factors such as HuR and its downstream targets such as Klf2, eNOS and BMP4 in PVECs exposed to parallel and disturbed flow in vitro and in lung tissues of rats with LHD developed after constriction of ascending aorta. Our study reveals the activation of HuR and downregulation of Klf2 and upregulation of BMP4 and CTGF. We also found that endothelial characters are lost in PVECs when exposed to disturbed flow and the cells display a smooth muscle like phenotype. On analyzing the lung tissues of rats with LHD we could find that the markers of En-MT were upregulated. Results of our study suggest that the disturbed flow could change the PVECs into a pro-inflammatory and proliferative phenotype leading to pulmonary vascular remodeling. PVEC dysfunction developed due to chronic exposure to disturbed flow in pulmonary circulation may be a reason for pulmonary vascular wall remodeling and $\mathrm{PH}$ in $\mathrm{PH}-\mathrm{LHD}$.

\section{0}

\section{NUCLEAR MEMBRANES ETB RECEPTORS MEDIATE ET-1-INDUCED INCREASE OF NUCLEAR CALCIUM IN HUMAN ENDOCARDIAL ENDOTHELIAL CELLS}

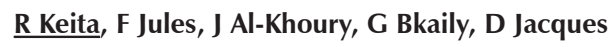

Department of Anatomy and Cell Biology, Faculty of Medicine, Université de Sherbrooke, Sherbrooke, Quebec

In human left ventricular endocardial endothelial cells (EECLs), plasma membrane (PM) ET-1Rs were reported to mediate ET-1-induced increase of intracellular calcium [Ca]i, however, in right EECs (EECRs), this effect was mediated by ETAR. In the present study we determined if, as for the $\mathrm{PM}$, nuclear membranes (NMs) ET-1Rs activation in EECLs and EECRs induce an increase of nuclear calcium $([\mathrm{Ca}] \mathrm{n})$ and we verified whether this effect is mediated via the same type of receptor as in PM. Using the PM-perforated technique and real 3D confocal microscopy, our results show that, as in PM intact cells, addition of cytosolic ET-1 to nuclei of both cell types induced a concentration-dependent sustained increase of [Ca]n. The ETAR antagonist prevented the effect of ET-1 on [Ca]n only in EECRs. However, the effect of cytosolic ET-1 on [Ca]n was prevented by the ETBR antagonist in both EECRs and EECLs. In conclusion, NMs' ETAR and ETBR mediated the effect of cytosolic ET-1 on [Ca]n in EECRs whereas only NMs' ETBR activation mediated the effect of cytosolic ET-1 in EECLs. Thus, the type of NMs' receptors mediating the effect of ET-1 on $[\mathrm{Ca}] \mathrm{n}$ are different from those of the PM mediating the increase in [Ca]i. Supported by an NSERC grant. 
121

MICROBIOLOGICAL IDENTIFICATION IN THE INTENSIVE CARE UNIT OF THE CITY OF ARACAJU, SERGIPE-BRAZIL

FLSG Santos ${ }^{1}$, SL Matos ${ }^{1}$, JB Pereira Jr ${ }^{1}$, DG Moura Neto ${ }^{1}$,

IMF Lobo ${ }^{2}$, SS Leão ${ }^{3}$, TMA Rodrigues ${ }^{1}$

${ }^{1}$ Molecular Anatomy Group, Morphology Department, Sergipe

Federal University, São Cristóvão; ${ }^{2}$ Urgency Hospital, Avenida Tancredo Neves, S/N Capucho, Aracaju, Sergipe; ${ }^{3}$ Department of Pathology, São Paulo Federal University, São Paulo, Brazil

In recent years there has been an increase in the number of antibiotic resistant microorganisms, particularly Gram-negative as Pseudomonas aeruginosa and Acinetobacter baumannii. High rates of infection caused by these microorganisms are responsible for high morbidity and mortality, failure of drug therapy, increased hospital stay and consequently the financial impact on the health system. However, while the occurrence of these bacteria to configure a public health problem, numerous studies show there is scarce information about the resistance genes present in multiresistant bacteria. This reality associated with the negative impact on the society, justifies the importance of identifying the expressed resistance genes in Acinetobacter baumannii and Pseudomonas aeruginosa resistant to carbapenems isolated from patients at a public hospital in northeastern Brazil. It was an experimental ecological study, prospective and quantitative. The demographic and clinical data of patients were being conducted through a specifically designed form. Multiplex PCR technique was performed for identification of resistance genes of Acinetobacter baumannii (imp bla, bla came, yes bla, bla Oxa-51, bla Oxa-58, bla bla Oxa-23 and Oxa-24) and Pseudomonas aeruginosa ( spm bla, bla bla came and imp). Descriptive analysis was performed using position and measures of variability for continuous variables and simple frequency for categorical variables. For the analysis of gene extraction amplification of resistance markers, we used GraphPad Prism version 5.0. The sample consisted of 17 patients, most men $(11-64.8 \%)$, median 40 years. Eleven $(64.8 \%)$ were from the responsiveness of HUSE-nine (53\%) were surgical. Most patients (14-82.3\%) were using five concurrent invasive devices with longer hospital stay for gastrostomy (74.2 days). The most commonly used antimicrobial was meropenem (76.4\%), which showed $100 \%$ sensitivity and colistin. Multiplex PCR was performed on 26 samples 7 Acinetobacter baumannii and Pseudomonas aeruginosa. Eighteen (69.2\%) strains of Acinetobacter baumannii showed the OXA-51 and bla bla genes OXA-23 co-eight (30.8\%), bla OXA-51. Only one Pseudomonas aeruginosa strain showed no resistance gene, while the other confirmed the presence of the bla gene spm.

Acknowledgements: The authors say thanks to the team workers of the Infected Disease Control Unit from Urgency Hospital, Health Foundation, Heathy Care Sergipe State Secretary.

\section{2}

\section{THE RENAL NERVES DECREASE RENAL BLOOD FLOW VARIABILITY BY BUFFERING ARTERIAL PRESSURE OSCILLATIONS IN CONSCIOUS RABBITS}

\section{AM Schiller, PR Pellegrino, IH Zucker}

Department of Cellular and Integrative Physiology, University of Nebraska Medical Center, Omaha, Nebraska, USA

Recent studies have focused on the therapeutic potential of renal denervation (RDNV) in many pathologies, but the physiological role of the renal nerves in the regulation of beat-to-beat hemodynamics is poorly characterized. We hypothesized that the renal nerves physiologically regulate beatto-beat renal blood flow variability (RBFV). This was tested in chronically instrumented rabbits that underwent either bilateral surgical renal denervation $(\mathrm{DDNx})$ or a sham denervation procedure (INV). Artifact-free segments of renal blood flow from calmly resting conscious rabbits were used to extract beat-to-beat RBFV for time-domain, frequency-domain, and nonlinear analysis. Compared to INV rabbits, DDNx rabbits had greater RBFV quantified as the standard deviation of RBFV and total spectral power. Transfer function analysis showed greater APV-RBFV gain in DDNx rabbits than in INV rabbits. The INV rabbits exhibited a baroreflex signature in the APV-RBFV transfer function which was absent in DDNx rabbits. In this same frequency band, coherence and fractional admittance gain were lower for INV rabbits, indicating frequency-specific modulation of RBFV by the renal nerves. RDNV did not affect glomerular filtration rate or extracellular fluid volume. These data suggest that the renal nerves decrease RBFV via baroreflex-mediated buffering of the renal circulation from changes in systemic arterial pressure.

Supported by PO1 HL62222.

\section{3}

\section{CELL-DEPENDENT LEVELS AND DISTRIBUTION OF NADPH OXIDASES}

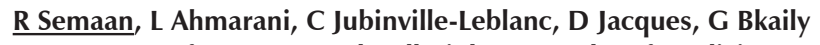
Department of Anatomy and Cell Biology, Faculty of Medicine, Université de Sherbrooke, Sherbrooke, Quebec

The objective of this work is to test the hypothesis that NADPH oxidases (NOXs) levels are different in human endocardial endothelial cells (hEECs), vascular smooth muscle cells (hVSMCs), and vascular endothelial cells (hVECs) and that NADPH oxidase-3 (NOX3) exists in these cell types. Using real 3D confocal microscopy and Western blot our results showed that the levels of NOX1 are similar in hVECs and hVSMCs, but lower in hEECs. NOX2 levels were higher in hVECs and lower in hVSMCs. NOX3 levels were the same in hVECs and hVSMCs, but lower in hEECs. NOX4 levels were similar in all cell types. NOX4 levels were higher in hVECs than in hVSMCs. NOX5 was also present throughout the 3 cell types, in the following order: hEECs $>$ hVSMCs $>$ hVECs. The level of basal ROS was highest in hVECs and lowest in hVSMCs. However, the $\mathrm{Ca} 2+$ level was highest in hVSMCs and lowest in hVECs. These findings suggest that all types of NOXs exist in hEECs, hVECs, and hVSMCs, although their density and distribution are cell-type dependent. The density of the different NOXs correlated with the ROS level, but not with the $\mathrm{Ca} 2+$ level. In conclusion, NOXs, including NOX3, exist in cardiovascular cells and their nuclei. The nucleus is a major source of ROS generation. Supported by CIHR.

\section{4}

\section{A PROTECTIVE ROLE OF IL-1 BETA IN ABDOMINAL AORTIC ANEURYSMS}

M Suh $^{1}$, J Carson ${ }^{1}$, W Xiong1 ${ }^{1}$ M Dale ${ }^{2}$, T Meisinger ${ }^{1}$, BT Baxter ${ }^{1}$

${ }^{1}$ Department of Surgery and ${ }^{2}$ Department of Pathology and Microbiology, University of Nebraska Medical Center, Omaha, Nebraska, USA

OBJECTIVES: Abdominal aortic aneurysm (AAA) is a chronic disease characterized by persistent inflammation maintained by an array of inflammatory cells and mediators. Interleukin 1 beta (IL-1 $\beta$ ) has largely been considered a pro-inflammatory cytokine but, interestingly, our previous studies of human AAAs showed that patients with AAAs have a decreased serum levels of IL-1 $\beta$ compared to matched control patients without AAAs. This led us to hypothesize that IL-1 $\beta$ could have a protective role in preventing the development of AAA.

METHODS: Abdominal aortic aneurysms were induced in IL-1 receptor $\mathrm{KO}$ (IL-1R-/-) and control mice using the $\mathrm{CaCl} 2$ murine model of AAA. Mice were sacrificed six weeks after aneurysm induction. Aortic diameters were measured and compared at the time of aneurysm induction and sacrifice. Aortic samples were further evaluated histologically using connective tissue staining to assess the aortic structure in both groups.

RESULTS: At the time of sacrifice, IL-1R-/- mice demonstrated a $73.9 \pm$ $3.3 \%$ increase in aortic diameter compared to a $25.9 \pm 5.8 \%$ increase in wild type control mice $(\mathrm{p}<0.05)$. On histology, IL-1R-/- mice had more severe disruption of the aortic architecture compared to control mice. These data suggest that in the absence of IL- $1 \beta$ signaling, mice are more susceptible to aneurysms and develop more severe pathology.

Conclusion: The current mouse data, in combination with our previous human studies, suggest a protective role for IL-1 $\beta$ in abdominal aortic aneurysm. These results are unexpected given that related pro-inflammatory cytokines TNF- $\alpha$ and IFN- $\gamma$ both promote aneurysm formation. 


\section{5}

EARLY POST-ISCHEMIC BLOOD-BRAIN BARRIER (BBB) DISRUPTION IN OBESITY

\section{H Sun, C Li, Z Jiang}

Cellular Biology \& Anatomy, Louisiana State University Health Sciences Center, Shreveport, Louisiana, USA

We determined the effect of high fat diet (HFD)-induced obesity on early BBB disruption following transient focal cerebral ischemia. Male C57BL/6J mice were fed a HFD or standard chow for 16 weeks. A cranial window was prepared over the left frontal, parietal and temporal cortex. Transient focal cerebral Ischemia was induced by directly ligating the middle cerebral artery (MCA) for two hours. Early BBB disruption was assessed by measuring Evans Blue and sodium fluorescein extravasation at 3 hours of reperfusion. The body weight was significantly increased in obesity group $(49.2 \pm 0.4 \mathrm{~g})$ compared to control group $(31.9 \pm 0.6 \mathrm{~g})$. There was no significant difference in conscious blood pressure and fasting blood glucose between control and obesity groups. Transient focal cerebral ischemia produced an early BBB disruption in both control and obesity groups. However, the magnitude of early BBB disruption was significantly greater in obesity group compared to control group. Topical treatment with L-NPA, 7-NI (neuronal nitric oxide synthase inhibitors) or L-NAME (a nonspecific nitric oxide synthase inhibitor) completely abolished the BBB disruption in control group, but only partially suppressed the BBB disruption in obesity group. Interestingly, matrix metallopeptidase (MMP)-9 activity of cerebral cortex was reduced in obesity group either under basal conditions or following ischemic stroke. Our findings suggest that obesity exacerbates early post-ischemic BBB disruption via a mechanism that appears to be unrelated to MMP-9 or NOS.

\section{6}

PDGF-BB INDUCES PHOSPHORYLATION OF POLO-LIKE KINASE-1 TO POTENTIATE PROLIFERATION OF SMOOTH MUSCLE CELLS IN HUMAN SAPHENOUS VEIN: POTENTIAL IMPLICATION IN VEIN-GRAFT DISEASE

\section{S Sur, S Chen, JT Sugimoto, DK Agrawal}

Creighton University School of Medicine, Omaha, Nebraska, USA

Coronary artery bypass grafting $(\mathrm{CABG})$ is the choice of procedure in patients with multi-vessel or left main coronary artery disease. Patency of saphenous vein graft (SVG) significantly declines following surgery compared to internal mammary artery (IMA). Intimal hyperplasia is the key event in SVG failure. PDGF-BB is a major growth factor released at the site of graft injury. Here, we examined, for the first time, the expression of PLK1 and pPLK1 in isolated human SV and IMA conduits that were freshly collected, SMCs isolated and cultured. In cultured SMCs, effect of PDGF-BB was examined on total PLK1, pPLK1, CDC2 and pCDC2 by Western blot analysis. Cell proliferation was measured using cell count and immunoblotting against the phosphorylated histone $\mathrm{H} 3$ tail. We found significantly higher expression of pPLK1, total PLK1, CDC2 and pCDC2 in PDGF-stimulated SV SMCs than IMA. These data suggest a greater and sustained sensitivity of SV SMCs to PDGF-BB-induced PLK1 activity than in IMA. A PLK1 inhibitor attenuated PDGF-induced proliferation in both IMA and SV SMCs. This could explain the development of intimal hyperplasia in SV conduits compared to IMA following CABG. Thus, inhibition of PLK1 could be a target in developing better therapeutic approach to prevent vein-graft disease.

Supported by NIH R01HL090580 grant

\section{7}

THE PROPENSITY OF VITAMIN D SUPPLEMENTATION TO REDUCE CHARACTERISTICS OF INFLAMMATION AND PROLIFERATION CAUSED BY CORONARY ARTERY INTERVENTIONAL PROCEDURES

VJ Swier Mosher, L Tang, MM Radwan, DK Agrawal Center for Clinical and Translational Science, Creighton University, Omaha, Nebraska, USA

Neointimal formation and cell proliferation resulting into in-stent restenosis is a major pathophysiological event following interventional coronary artery procedures. In this study, we examined the inflammatory profile and smooth muscle cell proliferation of microswine fed a high cholesterol diet. Swine were placed into one of three groups: a vitamin D supplemental diet (3000 to $4000 \mathrm{IU})$, a vitamin D sufficient diet (1000 to $2000 \mathrm{IU})$, or a vitamin $\mathrm{D}$ deficient diet. After six months of the high cholesterol diet, PTCA was performed in the LCX and bare mental stent implantation in the LAD for each swine. After a year of the diet, swine were euthanized and coronary arteries were embedded in methyl methacrylate or paraffin; and sections were stained with H\&E, trichrome, and Movat's pentachrome. The expression of Ki67 (proliferation marker), HMGB1 (inflammation and necrosis marker), TLR2 (monocyte and T-cell marker), and TLR4 (leukocyte marker) was evaluated by immunofluorescence. We continue to find a greater inflammation profile and a greater number of proliferating cells in the Vitamin D Deficient swine based on histological staining and immunoreactivity to HMGB1, TLR2, TLR4, and Ki67 in both PTCA LCX arteries and stented LAD arteries. This inflammation and proliferating profile decreases with increasing levels of Vitamin D, we find an even distribution of smooth muscle cells based on histological staining and only a few cells were immunopositive to HMGB1 and Ki67 in the Vitamin D Supplemental LCX and only cells surrounding the stent struts were immunopositive to TLR2 and Ki67 in the stented LAD arteries.

\section{8}

\section{CARBOTHERA IN THE TREATMENT OF ISCHEMIC FOOT} ULCERS: CASE STUDIES

H Hasebe, H Kumamoto, T MacInnis ${ }^{1}$, T Gregor ${ }^{1}$, PS Tappia ${ }^{1}$, GN Pierce

Mitsubishi Rayon Cleansui, Tokyo, Japan; and ${ }^{1}$ Asper Clinical Research Institute, ${ }^{2}$ St Boniface Hospital Research, Winnipeg, Manitoba

Foot ulceration usually precedes more serious foot complications such as infection, gangrene or amputation. The therapeutic potential of $\mathrm{CO}_{2}$ enriched water on treating foot ulcers is being evaluated. In the two case studies, a 57-year old male and a 72-year old female with diabetes, peripheral arterial disease and undergoing hemodialysis for end-stage renal disease presented with one non-infected ischemic arterial foot ulcer. Foot bathing in $\mathrm{CO}_{2}$-enriched water (1000-1200 ppm) at $37 \pm 0.5^{\circ} \mathrm{C}, 3 /$ week for 15 min for 3 months resulted in a significant healing of the wound that was associated with an increase in $\mathrm{O}_{2}$ saturation levels of the wound area, improved blood flow as evidenced by increases in ankle-brachial index, reduction in fasting blood glucose levels, and a small decrease in HbA1c. $\mathrm{CO}_{2}$ foot bathing could help to accelerate the wound healing process.

\section{9}

\section{GENERATION OF HUMAN INDUCED PLURIPOTENT STEM CELLS WITHOUT INTEGRATION AND TRANSGENE UNDER FEEDER-FREE CONDITIONS}

\section{Y-T Xuan, O-L Wang, Y Zhu, B Dawn}

Division of Cardiovascular Diseases, Cardiovascular Research Institute, Midwest Stem Cell Therapy Center, University of Kansas Medical Center, Kansas City, Kansas, USA

Human induced pluripotent stem cells (hiPSCs) have immense potential for cardiac repair. However, for translational purposes, it is critical to generate clinical-grade hiPSCs under viral integration-free feeder-free conditions. We examined the efficacy of hiPSC generation from human skin fibroblasts using an episomal reprogramming method.

Human fibroblasts were transfected with episomal vectors expressing transcription factors Oct4, Sox2, Nanog, Lin28, Klf4, and L-Myc (Combination 1) or Oct4, Sox2, Nanog, Lin28, Klf4 (Combination 2) via electroporation. Because the proto-oncogene product c-Myc markedly increases tumor formation in iPSC-derived chimeric mice, c-Myc was replaced with L-Myc (less tumorigenic) in Combination 1 and was omitted in Combination 2 to limit the incidence of tumor formation. Transfected fibroblasts were then plated to vitronectin-coated 6-wells in fibroblast culture medium and $24 \mathrm{~h}$ later the medium was changed to chemically defined N2B27 medium containing inhibitors of GSK3 $\beta$, MEK, TGF- $\beta$ receptor, and ROCH and human leukemia inhibitory factor. Emerged iPSC colonies were readily picked between days 21 and 24 after transfection in Combination 1 and 
much earlier between days 17-21 in Combination 2. These cells highly expressed pluripotent cell surface antigens (SSEA-4, TRA-1-60, and TRA-1-81) and endogenous transcription factors (Oct4 and Sox2) and were able to differentiate into three germ layers in vitro. Taken together, these data demonstrate that these are pluripotent cells.
In summary, hiPSCs can be successfully generated from human skin fibroblasts using a non-integrating and non-viral episomal reprogramming method under feeder-free conditions. These hiPSCs are also completely free of vector and transgene. This method can be easily adapted to the production of clinical-grade human iPSCs for cardiac cell therapy.

\section{AUTHOR INDEX}

\section{A}

Abdel-Karim Abdel-Malak N .......... 095

Abo Alrob O ................... 063

Acsai K....................... 116

Adi N..................... 037

Afzal R...................... 011

Aghila Rani KG............. 010

Agrawal DK . . . . . . 013,071,087,091,093,101

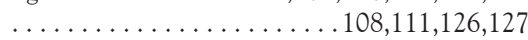

Agrawal SS. ................. 074

Ahmarani L . . . . . . . . . . . . . . . . . . . . 123

Ahmari N. .................... 069

Ajithkumar GS . . . . ............ 119

Aliani M ....................... 004

Al-Khoury J . . . . . . . . . . . . . 120

Almalki S.................... 111

Almalki SG ................... 091

Apparsundaram S. . . . . . . . . . . . 023

Ardell JL. . . . . . . . . . . . . . . . . . . . . . 025

Arias AJ . . . . . . . . . . . . . . . . . . . . 114

Arige V.................... 060,113

Arrick DM .................... 003

Auger-Messier M . . . . . . . . . . . . . . . 098

Aukema H . . . . . . . . . . . . . . . . . . . . .004,094

\section{B}

Baba SP .......................................... 092

Baczkó I . . . . . . . . . . . . . . . . 057

Bagchi RA . . . . . . . . . . . . . . 088,115

Bahl A . . . . . . . . . . . . . . . . 060,113

Baxter BT. . . . . . . . . . . . . . . 099,124

Becker BK . . . . . . . . . . . . . . . 015

Bhatnagar A. ..................... 092

Binil Raj SS . . . . . . . . . . . . . . . . . 119

Bkaily G............. 095,120,123

Blackman AO .................... 049

Bolli R ....................... 009

Boosani CS .................... 093

Bösze Z . . . . . . . . . . . . . . . . 057

Bouchard DR ................... 104

Bowen J . . .................. 037

Bratasz A . . . . . . . . . . . . . . . . . 070

Britton SL ..................... 040

Buttar HS...................... 075

\section{$\mathrm{C}$}

Caligiuri S . . . . . . . . . . . . . . . . . 004,094

Carpentier A . . . . . . . . . . . . . . . . . 098

Carson J ....................... 124

Carson JS . . . . . . . . . . . . . . . . . . . 099

Carvajal JM ...................... 069

Case AJ ..................... 032

Castañeda Saldaña E ............... 061

Chacko G..................... 072

Chamoun M.................... 095

Chao J ..................... 036

Chaturvedi P . . . . . . . . . . . . . . 014,109

Chaudhary R . . . . . . . . . . . . . . 112

Chen S. . . . . . . . . . . . . . . . . . 087,126
Chernyavskiy I. . . ................... 096

Clemmer J . . . . . . . . . . . . . . . . . . . . 097

Conklin D . . . . . . . . . . . . . . . . . . 092

Couture EL. . . . . . . . . . . . . . . . . . . 098

Csepe TA . . . . . . . . . . . . . . . . . 059,070

Cui M-Z..................... 076

Czirok A..................... 084

Czubryt MP . . . . . . . . . . . . . . 088, 115

\section{D}

D’Orléans-Juste P. . . . . . . . . . . . . 031,078

Dai Y ........................ 054

Dale M. . . . . . . . . . . . . . . . . . . . . 099, 124

Danielle J . . . . . . . . . . . . . . . . . 090

Dawn B ................ 011,084,129

Deka PK..................... 100

Del Rio R.................... 055

Desbiens L . . . . . . . . . . . . . . . . . 078

Desouza C. . . . . . . . . . . . . . . . . . 037

Dewan A ........................................... 033

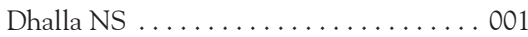

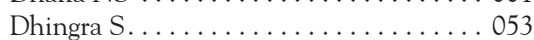

Dibrov E..................... 004

Ding H...................... 033

Ding Z . .................... 054

Djossou KP................... 101

Duhamel TA .................... 104

Dunlap ME. .................. 066

\section{E}

Edel AL ........................ 004

Edye-Mazowita A. . . . . . . . . . . . . . 104

\section{$\mathrm{F}$}

Familtseva A ....................................... 014

Faraci FM...................... 002

Farand P . . . . . . . . . . . . . . . 098

Fedorov VV . . . . . . . . . . . . . . . . 059,070

Felder RB . . . . . . . . . . . . . . . . . . . 008

Feng Y .................... 035

Ferrario CM ..................... 005

Fillmore $\mathrm{N} \ldots \ldots \ldots \ldots \ldots \ldots \ldots . \ldots 102$

Fukushima A ................. 102

\section{G}

Ganguly R ..................................... 036

Gao J .................... 036

Gao L . . . . . . . . . . . . . . . . . . . . . 036

Garcia-Pereira F. . . . . . . . . . . . . . . 069

Gembardt F ................ 036

Ghassan B ............................................. 060

Gomes $\mathrm{O} \ldots \ldots \ldots \ldots \ldots \ldots \ldots 65$

Gomes OM. . . . . . . . . . . . . . . . . . . 049

Goswami SK . . . . . . . . . . . . . . 113

Goyal RK..................... 023

Gregor T. ................... 128

Grobe JL....................... . 020

Grueter CE. .................. 086

Guo Y...................... 092
Gupta A....................... 074

Gupta MP ................... 042

Guzman R .................. 004,094

Gy Papp J .................. 116

\section{$\mathrm{H}$}

Haack KK. . . . . . . . . . . . . . . . . 117

Hackfort BT. . . . . . . . . . . . . . . 103,118

Hall DD . . . . . . . . . . . . . . . . . . 086

Hamm NC. . . . . . . . . . . . . . . . . . . 104

Hansen BJ . . . . . . . . . . . . . . . . . . . 059,070

Hao F . . . . . . . . . . . . . . . . . . 076

Hasanally D . . . . . . . . . . . . . . . . . . 112

Hasebe H . . . . . . . . . . . . . . . . . 046,128

Hawkins D . . . . . . . . . . . . . . . 046

Hayashi H ..................... 105

Hester R. . . . . . . . . . . . . . . . . . 097

Higgins RSD . . . . . . . . . . . . . 070

Hiripi L . . . . . . . . . . . . . . . . . . . 057

Hoetker D . . . . . . . . . . . . . . . . . . 092

Hong B.................... 050

Hornyik T . . . . . . . . . . . . . . . . 057

Houde M . . . . . . . . . . . . . . . 031,078

Hristova K . . . . . . . . . . . . . . . . . . 082

Hummel JD .................... 070

I

Ikhapoh IA ................ 013

Isai DG. ..................... 084

\section{$\mathrm{J}$}

Jacques D . . . . . . . . . . . . . 095,120,123

Janssen PML .................. 070

Jayne LA . . . . . . . . . . . . . . . . . . . . 070

Jeevanantham $V \ldots \ldots \ldots \ldots \ldots \ldots . \ldots 11$

Ji H....................... 019

Jiang Z . . . . . . . . . . . . . . . . . . 125

Johnson AJ...................... 104

Johnson AM ...................... 010

Johnson RD . . . . . . . . . . . . . . . . . . 069

Johnson TA . . . . . . . . . . . . . . . 029,106

Johnstone DE. . . . . . . . . . . . . . . . 063

Johny A-K . . . . . . . . . . . . . . . . . . . 090

Joseph J. . . . . . . . . . . . . . . . . . . 069

Jost N . . . . . . . . . . . . . . . . . 116

Ju JC.................... 107

Jubinville-Leblanc C . . . . . . . . . . . . . 123

Juhász V . . . . . . . . . . . . . . . . . . . . 057

Jules F...................... 120

\section{K}

Kalani A................................ 014 Kalyanasundaram A................ 070

Kamoi D...................... 105

Kartha CC . . . . . . . . . . . . . 010,052,119

Kasuga $\mathrm{H} \ldots \ldots \ldots \ldots \ldots \ldots \ldots \ldots$

Kawahara H.................... 105

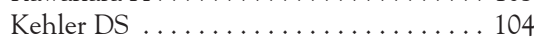

Keita R.......................... 120 
Khanna S..................... 113

Khullar M................. 060,113

Kilic A ....................... 070

Kim S........................ 069

Kimber DE ....................... 104

Koch LG ................... 040

Kohajda Z. ................... 116

Kokouvi P . . . . ................ 101

Kormos A..................... 116

Kosa E ....................... 084

Kouassi T ................... 108

Kukreja C...................... 073

Kukreja RC .................. 044

Kumamoto H. . . . . . . . . . . . . . 046,128

Kumar A...................... 060

Kundu S...................... 017

Kunkel GH ...................... 109

Kutryk MJB ................. 068

Kutty S...................... 083

$\mathrm{L}$

Leão SS ........................ 121

Lecomte R . . . . . . . . . . . . . . . . . 031

Lefnaier W................... 016

Lepage $M \ldots \ldots \ldots \ldots \ldots \ldots \ldots . \ldots . \ldots 31$

Levijoki J . . . . . . . . . . . . . . . . . . . 116

Li C . . . . . . . . . . . . . 110,125

Li N ........................ 070

Li R-K ........................ 012

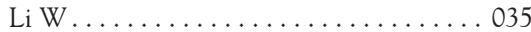

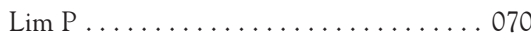

Lindsey ML . . . . . . . . . . . . . 051

Liu X . . . . . . . . . . . . . . . . . . 019,034

Llamas Y. . . . . . . . . . . . . . . . . 091,111

Lobo IMF . ................... 121

Lohmeier TE .................... 027

Lopaschuk GD . . . . . . . . . . . . . 063,102

Lu J........................... 022

Lu S ....................... 097

\section{M}

MacInnis T . . . . . . . . . . . . . . . . 128

Maddaford TG . . . . . . . . . . . . . . 112

Mahapatra N ................... 113

Mahapatra NR................... 060

Major P .................... 057

Manavalan V.................. 054

Marcus NJ .................... 055

Matos SL ..................... 121

Mayhan WG ................ 003,110

McGavock J . . . . . . . . . . . . . . . . . . . . . 104

McManus B . . . . . . . . . . . . . . . . 062

Mediratta J................... 055

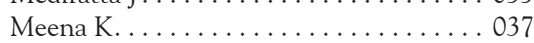

Mehta JL . . . . . . . . . . . . . . 022,054

Meisinger T . . . . . . . . . . . . . . . 124

Metreveli N . . . . . . . . . . . . . . . . . 014

Mishra PK ........ 030,034,045,103,118

Mittal A................... 060,113

Mittwede P..................... 097

Mohler PJ........................ 070

Moore BT..................... 070

Mori-Llontop LM ................. 114

Moriyama Y ................... 105

Moulton MJ . .................. 050

Moura Neto DG. ................ 121

\section{$\mathrm{N}$}

Nagalingam RS ................. 115

Nagib El-kilany GE ............... 080

Nagy N..................... 116

Nánási PP. . . . . . . . . . . . . . . . . . . 056

Nandi SS . . . . . . . . . . . . . . . . . 030, 034,118

Nguyen M . . . . . . . . . . . . . . . . . . 098

Norman ME....................... 104

\section{$\mathrm{O}$}

Oravecz K.................... 116

Ostadal B . . . . . . . . . . . . . . . . . . . 018

Oudit GY................... 06

\section{P}

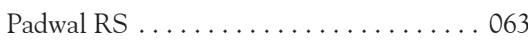

Parikh S...................... 072

Parthasarathy S . . . . . . . . .............. 006

Patel KP . . . . . . . . . . . . 034,038,063

Pathak D ...................... 100

Pelham CJ ...................... 013

Pellegrino PR . . . . . . . . . . . 117,122

Pereira Jr JB . . . . . . . . . . . . . . . . . 121

Perriotte-Olson C . . . . . . . . . . . . . 037

Phil M ..................... 039

Pierce G........................ 094

Pierce GN . . . . . . . . . . . . . . . . 004,112,128

Pillai V....................... 042

Pollesello P. ..................... 116

Powell KA . . . . . . . . . . . . . . . . . . . . 070

Powers JC. . . . . . . . . . . . . . . . . . 072

Pozehl B..................... 041

Pozehl BJ ........................ 100

Prasad R..................... 060

Prathipati P . . . . . . . . . . . . . 103,118

Provost C . . . . . . . . . . ........ 095

Pügge C . . . . . . . . . . . . . . . . 055

Pushpakumar S ................. 014

Qi Y ..................

\section{$\mathrm{R}$}

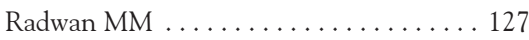

Rai V ......................... 071

Raizada MK . . . . . . . . . . . . . . . . . . . 069

Rajasingh J. . . . . . . . . . . . . . . . 084

Rajasingh S ................... 084

Ramachandran S . . . . . . . . . . . . 052

Ramalingam R.............................. 037

Ramasamy R................... 079

Rana S .................... 060,113

Rao VH ................... 071

Raut SK . . . . . . . . . . . . . . . . . 060

Ravandi A . . . . . . . . . . . . . . . . . 004,094,112

Ready AE........................ 104

Recchia FA ................ 064,072

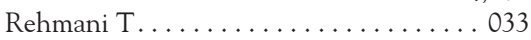

Ricapito MP.................... 098

Roche PL . . . . . . . . . . . . . . . . 088, 115

Rodrigues TMA . . . . . . . . . . . . . . . 121

Rodriguez-Leyva D. . . . . . . . . . . . . . 004,094

Rosas ME . . . . . . . . . . . . . . . . . . . 114

Rudman A . ................... 072

Ruhlman MK.................... 099

\section{$\mathrm{S}$}

Sabri A...................... 072

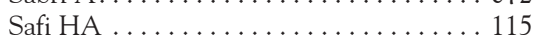

Saikia UN . . . . . . . . . . . . . . . . . . . . 060

Salih M .................. 016,033

Samant S . . . . . . . . . . . . . . . . . 042

Samanta A.................... 011

Samanta S . . . . . . . . . . . . . . . . 084

Sandberg K. . . . . . . . . . . . . . . . . . . . . 019
Sanjay G. . . . . . . . . . . . . . . . . . 119

Sankaralingam S . . . . . . . . . . . 063

Santisteban MM .................. 069

Santos FLSG . . . . . . . . . . . . 121

Saraswathi V . .................. 037

Sarkar S . . . . . . . . . . . . . . . . . . . 060,113

Schiller AM . . . . . . . . . . 055,117,122

Schultz HD ................. 028,055

Schwertani AG ................ 031

Seclén-Santisteban SN . . . . . . . . . . . 114

Secomb TW.................. 050

Seki M ..................... 072

Semaan R.................... 123

Shahshahan HR. . . . . . . . . . . . . . . 118

Shaikh NA................... 077

Sharma AM .................... 063

Sharma G..................... 074

Sharma NM . . . . . . . . . . . . . . . . 034

Sharma R . . . . . . . . . . . . . . . . . . 060,113

Sharma S ................... 060

Shenoy V . . . . . . . . . . . . . . . . . . . . . . 069

Shivkumar K . . . ................ 067

Simon Y........................ 095

Simonetti O................... 070

Singla D . . . . . . . . . . . . . . . . . . 089

Singla DK. . . . . . . . . . . . . . . . . . 029, 106

Sobral Neto J . . . . . . . . . . . . . . . . . . . 049

Sozzi FB . . . . . . . . . . . . . . . . . . 081

Spitler K. . . . . . . . . . . . . . . . . . 086

Stammers AN .................... 104

Stephen S. . . . . . . . . . . . . . . . 107

Stoupa S. .................... 071

Strachan SM .................... 104

Sugimoto JT. . . . . . . . . . . . . . 126

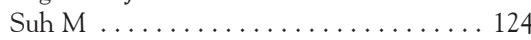

Sun H. . . . . . . . . . . . . . 110,125

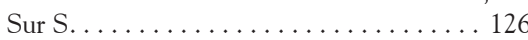

Suzuki K...................... 047

Swier Mosher VJ . . . . . . . . . . . . . . . . 127

\section{$\mathrm{T}$}

Takashi I. ..................... 107

Tang L . . . . . . . . . . . . . . . . 127

Tappia PS................... 128

Thorp E .................... 085

Tian J . . . . . . . . . . . . . . . . . . . . 032

Tóth A....................... 116

Tremblay A ................... 031

Tremblay L. . . . . . . . . . . . . . . . . . . 031,098

Tricot B . . . . . . . . . . . . . . . . . . . 098

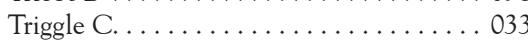

Tsai EJ .................... 072

Tuana BS . . . . . . . . . . . . . 016,033,043

Turan B .................... 048

Tyagi SC. . . . . . 0. 014,017,039,096,109

\section{$\mathrm{V}$}

Varga R........................ 057

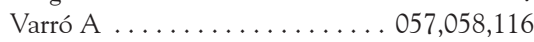

Veeranki S . . . . . . . . . . . . . . . . . 017,096

Vinitha A.........................................

Virág L . . . . . . . . . . . . . . . . . . . . 116

W
Wagg CS ................ 063,102

Walther T........................ 036

Wang H ...................... 026

Wang H-J . . . . . . . . . . . . . . . . . . 015

Wang O-L .................... 129

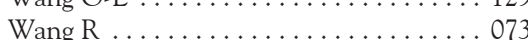

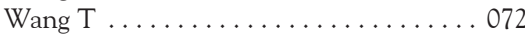


Author Index

Wang X . . . . . . . . . . . . . . 022,054

Weighell W .................... 004

Weiss R. . . . . . . . .............. 070

Winchester L . . . . . . . . . . . . . . . . 096

Wittnich C. .................... 021

Wu D ................... 076

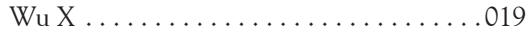

$\mathrm{X}$

Xi L ......................... 073

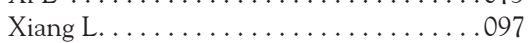

Xiong W. . . . . . . . . . . . . . . 099, 124

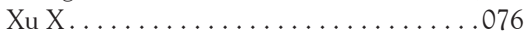

Xuan Y-T ......................... 129

\section{Y}

Yamada T........................105

Yang X-F.................. 007

\section{Z}

Zhang D ..................... 092

Zhang F ....................076
Zhang L . . . . . . . . . . . . . . . . . . . 102

Zhao J.......................... 070

Zheng H..................... 034,038

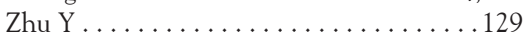

Zimmerman MC ...............032

Zingler MB. . . . . . . . . . . . . . . . . . . .069

Zou M-H .....................024

Zubcevic J.....................069

Zucker IH......... 015,036,055,117,122 